\title{
Palladium and Brønsted Acid Co-catalyzed Biginelli-like Multicomponent Reactions via in Situ-Generated Cyclic Enol Ether: Access to Spirofuran-hydropyrimidinones
}

Shuyan Yu, ${ }^{1 *}$ Jingxin Wu, ${ }^{1}$ Hongbing Lan, ${ }^{1}$ Lihong Gao, ${ }^{1}$ Hengyu Qian, ${ }^{1}$

\author{
Kaiqi Fan, ${ }^{1}$ Zhigang Yin $^{1 *}$ \\ ${ }^{1}$ Material and Chemical Engineering College, Zhengzhou University of Light Industry, \\ Zhengzhou 450002, Henan, P. R. China \\ yushuyan_zzuli@163.com \\ yinck@zzuli.edu.cn
}

\section{Table of Contents}

1. General information $\mathrm{S} 1$

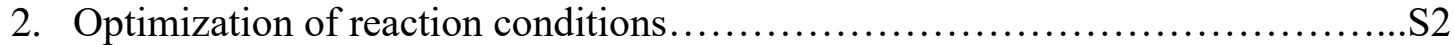

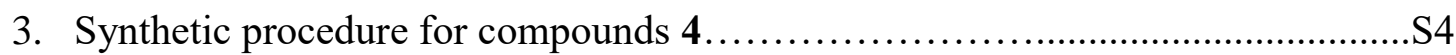

4. Reaction details of electron-deficient aromatic aldehydes $1 \mathrm{e}-1 \mathbf{h} . \ldots \ldots \ldots \ldots \ldots . . . . . . . .55$

5. X-ray crystal structures of representative compounds........................S10

6. Characterization data of new compounds.................................... 11

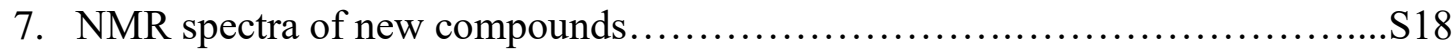

8. Enantioselective exploration and HPLC spectra..............................S43 


\section{General Information}

All the reactions were conducted without $\mathrm{N}_{2}$ protection and no dried manipulation of solvent was needed. Analytical thin-layer chromatography (TLC) was carried out on Merck 60 F254 pre-coated silica gel plate (0.2 $\mathrm{mm}$ thickness). Commercially available materials purchased from Alfa Aesar or Sigma-Aldrich were used as received. Column chromatography was performed on silica gel (300-400 mesh). Melting points were measured using a TY-60 microscopic apparatus. Proton nuclear magnetic resonance $\left({ }^{1} \mathrm{H}\right.$ NMR) and carbon nuclear magnetic resonance $\left({ }^{13} \mathrm{C}\right.$ NMR $)$ spectra were recorded on Bruker (400 MHz) spectrometer and Bruker Avance 500 $(500 \mathrm{MHz})$ spectrometer. Chemical shifts were recorded in parts per million (ppm, $\delta$ ) relative to tetramethylsilane $(\delta 0.00)$ or chloroform $\left(\mathrm{d}=7.26\right.$, singlet). ${ }^{1}$ HNMR splitting patterns are designated as singlet (s), doublet (d), triplet (t), quartet (q), dd (doublet of doublets), m (multiplets). All first-order splitting patterns were assigned on the basis of the appearance of the multiplet. Splitting patterns that could not be easily interpreted are designated as multiplet(m) or broad (br). High resolution mass spectral analysis (HRMS) was performed on Finnigan MAT 95 XP mass spectrometer (Thermo Electron Corporation). 


\section{Optimization of reaction conditions}

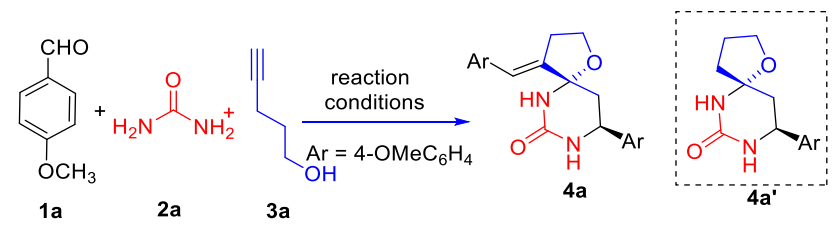

Screening of transition metals

\begin{tabular}{|c|c|c|c|c|}
\hline Entry & $\mathrm{TM}$ & $\mathrm{BA}$ & Solvent & Yield $^{b}$ \\
\hline 1 & $\mathrm{AgOTf}$ & TFA & DCE & $49 \%$ \\
\hline 2 & $\mathrm{Cu}(\mathrm{OTf})_{2}$ & TFA & DCE & NR \\
\hline 3 & $\mathrm{AuPPh}_{3} \mathrm{Cl}$ & TFA & DCE & trace \\
\hline 4 & $\mathrm{AuPPh}_{3} \mathrm{Cl}+\mathrm{AgOTf}$ & TFA & DCE & trace \\
\hline 5 & $\mathrm{AuCl}_{3}$ & TFA & DCE & $35 \%$ \\
\hline 6 & $\mathrm{PdCl}_{2}$ & TFA & DCE & $80 \%$ \\
\hline 7 & $\mathrm{Sc}(\mathrm{OTf})_{3}$ & TFA & DCE & NR \\
\hline 8 & $\mathrm{PtCl}_{4}$ & TFA & DCE & NR \\
\hline 9 & $\mathrm{PtCl}_{2}$ & TFA & DCE & $38 \%$ \\
\hline 10 & $\mathrm{Pt}(\mathrm{COD}) \mathrm{Cl}_{2}$ & TFA & DCE & $41 \%$ \\
\hline 11 & $\mathrm{Pt}(\mathrm{COD}) \mathrm{Me}_{2}$ & TFA & DCE & $29 \%$ \\
\hline
\end{tabular}

The reaction was performed with $\mathbf{1 a}(\mathbf{1} \mathrm{mmol}), \mathbf{2 a}(1.1 \mathrm{mmol}), \mathbf{3 a}(1.2 \mathrm{mmol})$ in the presence of transition metal (10 $\mathrm{mol} \%)$ and TFA (20 mol\%) in $3 \mathrm{mLDCE}$ at $50^{\circ} \mathrm{C}$ in $\mathrm{N}_{2}$; Isolated yield.

Screening of Brønsted acid

$\begin{array}{lllll}\text { Entry } & \mathrm{TM} & \mathrm{BA} & \text { Solvent } & \text { Yield } \\ 12 & \mathrm{PdCl}_{2} & \mathrm{TFA} & \mathrm{DCE} & 81 \% \\ 13 & \mathrm{PdCl}_{2} & \mathrm{HAc} & \mathrm{DCE} & 54 \% \\ 14 & \mathrm{PdCl}_{2} & \mathrm{PTSA} & \mathrm{DCE} & \text { complex } \\ 15 & \mathrm{PdCl}_{2} & \mathrm{HBF}_{4} & \mathrm{DCE} & \text { complex } \\ 16 & \mathrm{PdCl}_{2} & 1,1^{\prime} \text {-'Binaphthyl-2,2'-diyl } & \mathrm{DCE} & 38 \% \\ & & & \end{array}$

The reaction was performed with $\mathbf{1 a}(2 \mathrm{mmol}), \mathbf{2 a}(1.1 \mathrm{mmol}), \mathbf{3 a}(1.2 \mathrm{mmol})$ in the presence of $\mathrm{PdCl}_{2}(10 \mathrm{~mol} \%)$ and Brønsted acid (20 mol\%) in $3 \mathrm{~mL}$ DCE at $50^{\circ} \mathrm{C}$ in $\mathrm{N}_{2}$; Isolated yield.

Screening of solvents

$\begin{array}{lllll}\text { Entry } & \mathrm{TM} & \mathrm{BA} & \text { Solvent } & \text { Yield } \\ 12 & \mathrm{PdCl}_{2} & \mathrm{TFA} & \text { DCE } & 81 \% \\ 17 & \mathrm{PdCl}_{2} & \text { TFA } & \text { Dioxane } & 86 \%\end{array}$




$\begin{array}{lllll}18 & \mathrm{PdCl}_{2} & \text { TFA } & \text { THF } & 65 \% \\ 19 & \mathrm{PdCl}_{2} & \text { TFA } & \text { EtOH } & \text { trace } \\ 20 & \mathrm{PdCl}_{2} & \text { TFA } & \text { Toluene } & 74 \% \\ 21 & \mathrm{PdCl}_{2} & \text { TFA } & \text { AcOH } & 57 \% \\ 22 & \mathrm{PdCl}_{2} & \text { TFA } & \mathrm{CH}_{3} \mathrm{CN} & 49 \%\end{array}$

The reaction was performed with $\mathbf{1 a}(2 \mathrm{mmol}), \mathbf{2 a}(1.1 \mathrm{mmol}), \mathbf{3 a}(1.2 \mathrm{mmol})$ in the presence of $\mathrm{PdCl}_{2}(10 \mathrm{~mol} \%)$ and TFA (20 mol\%) in $3 \mathrm{~mL}$ solvent at $50^{\circ} \mathrm{C}$ in $\mathrm{N}_{2}$; Isolated yield.

\section{Screening of temperature and loading of catalysts}

$\begin{array}{lllll}\text { Entry } & \mathrm{PdCl}_{2}(\mathrm{~mol} \%) & \text { TFA }(\mathrm{mol} \%) & \text { Temp. }\left({ }^{\circ} \mathrm{C}\right) & \text { Yield (time) } \\ 17 & 10 & 20 & 50 & 86 \%(5 \mathrm{~h}) \\ 23 & 10 & 20 & 80 & 79 \%(2 \mathrm{~h}) \\ 24 & 10 & 20 & \mathrm{rt} & \text { slow }(>24 \mathrm{~h}) \\ 25 & 5 & 10 & 50 & \text { very slow }(>24 \mathrm{~h}) \\ 26 & 5 & 20 & 50 & 79 \%(17 \mathrm{~h})\end{array}$

The reaction was performed with $\mathbf{1 a}(2 \mathrm{mmol}), \mathbf{2 a}(1.1 \mathrm{mmol}), \mathbf{3 a}(1.2 \mathrm{mmol})$ in the presence of $\mathrm{PdCl}_{2}$ and TFA in $3 \mathrm{~mL}$ dioxane in $\mathrm{N}_{2}$; The reaction was monitored by TLC; Isolated yield.

\section{Control experiments}

$\begin{array}{lllll}\text { Entry } & \mathrm{TM}(1 \mathrm{omol} \%) & \mathrm{BA}(20 \mathrm{~mol} \%) & \text { Solvent } & \text { Yield } \\ 17 & \mathrm{PdCl}_{2} & \text { TFA } & \text { Dioxane } & 86 \% \\ 27 & \mathrm{PdCl}_{2} & -- & \text { Dioxane } & \mathrm{NR} \\ 28 & - & \text { TFA } & \text { Dioxane } & \text { trace } \\ 29^{a} & \mathrm{PdCl}_{2} & \text { TFA } & \text { Dioxane } & 87 \%\end{array}$

Unless noted, the reaction was performed with $\mathbf{1 a}(2 \mathrm{mmol}), \mathbf{2 a}(1.1 \mathrm{mmol}), \mathbf{3 a}(1.2 \mathrm{mmol})$ with indicated catalysts in 3

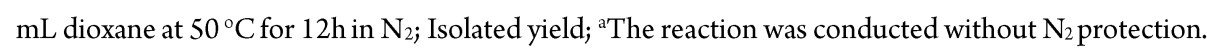




\section{Synthetic procedure for spirofuran-hydropyrimidinones compounds 4}

In a $10 \mathrm{~mL}$ round-bottom flask, aromatic aldehyde $\mathbf{1}(2 \mathrm{mmol})$, urea or thiourea $2(1.1 \mathrm{mmol})$ and 4-pentyl-1-ol 3 (1.2 mmol) were successively introduced into a solution of $\mathrm{PdCl}_{2}(10 \mathrm{~mol} \%)$ in dioxane $(3 \mathrm{~mL})$. Then trifluoroacetic acid $(20 \mathrm{~mol} \%)$ was added. After that the mixture was stirred at $50{ }^{\circ} \mathrm{C}$ with oil bath until aldehyde was fully consumed (determined by TLC analysis). In case of thiourea, the reaction temperature was $100^{\circ} \mathrm{C}$. The resulting mud was cooled to room temperature, neutralized with aqueous $\mathrm{NaHCO}_{3}$, and diluted with $30 \mathrm{~mL}$ DCM. After separation from aqueous phase, organic phase was washed with water and dried with $\mathrm{Na}_{2} \mathrm{SO}_{4}$. Removal of solvent under vacuum, the residue was purified by column chromatography on silica gel with $\mathrm{DCM} / \mathrm{MeOH}$ as elute (In case of thiourea, Hex/EA was used as elute). The desired spirofuran-hydropyrimidinones compound 4 was obtained with good yield and excellent diastereoselectivity. All the new products were characterized by ${ }^{1} \mathrm{H}$ NMR, ${ }^{13} \mathrm{C}$ NMR, HRMS and melt point and then their structures were unambiguously confirmed by X-ray analysis of Compound $\mathbf{4 a}, \mathbf{4 l}, \mathbf{4 m}, \mathbf{4 u}$ and $\mathbf{4 f}$ '. 


\section{Reaction details of electron-deficient aromatic aldehydes $1 \mathrm{e}-1 \mathrm{~h}$}

In the reactions of benzaldehydes with nitro- or ester- group, side products $\mathbf{4 e}$ '-4h'were isolated with 30\%-44\% yields, along with the desired products $4 \mathbf{e}-4 \mathrm{~h}$ with 46\%-57\% yields (Scheme S1). In order to investigate the reason for the formation of $\mathbf{4} \mathbf{e}^{\mathbf{\prime}} \mathbf{- 4 h}$ ', some control experiments were conducted. Firstly, compound 4f' was treated with the same catalytic conditions, but no transformation to $\mathbf{4 f}$ was observed even after $48 \mathrm{~h}$ (Scheme S2a). Then, the reactions of different amount of aromatic aldehyde 1f or 1e with urea and 4-penty-1-ol were explored (Scheme S2b and $\mathrm{S} 2 \mathrm{c}$ ). (Following the general synthetic procedure, the reaction was stirring at $50{ }^{\circ} \mathrm{C}$ with oil bath for $12 \mathrm{~h}$. After that, the mixture was quenched with aqueous $\mathrm{NaHCO}_{3}$, diluted with $\mathrm{DCM}$ and washed successively with water and brine. After evaporation of solvent, the residue was dissolved in DMSO- $d_{6}$ for ${ }^{1} \mathrm{HNMR}$ analysis.)

Scheme S1 the reactions of electron-deficient aromatic aldehydes $\mathbf{1 e - 1 h}$ under standard conditions

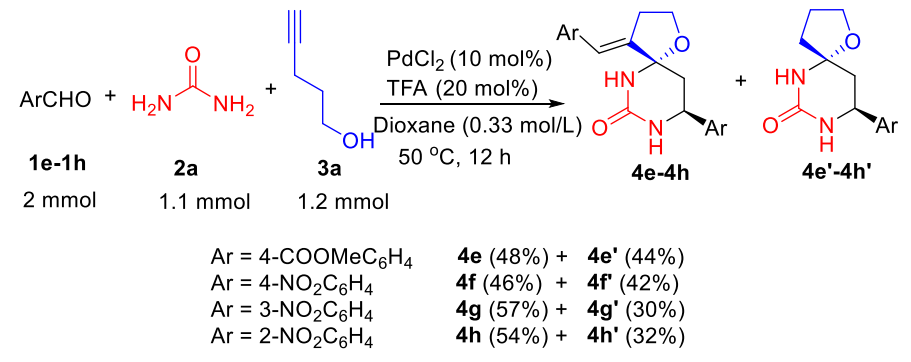

Scheme S2 Control experiments

a)
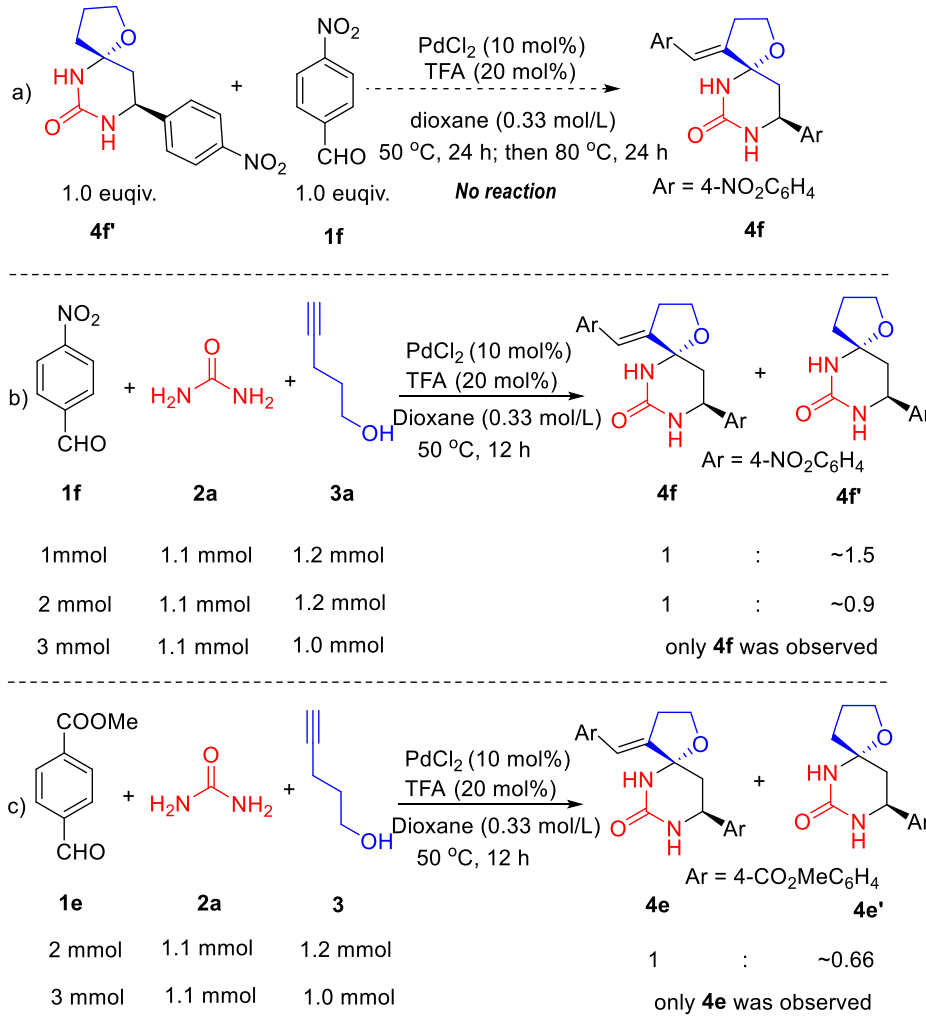
From the crude ${ }^{1}$ HNMR spectrum of reaction mixtures, the formation of $\mathbf{4 f}$ ' was found to be depressed as the loading of 4-nitrobenzaldehyde increased ( $1 \mathrm{mmol}, \mathbf{4 f} / \mathbf{4} \mathbf{f}^{\prime}=1.0: 1.5 ; 2 \mathrm{mmol}, \mathbf{4} \mathbf{f} / \mathbf{4} \mathbf{f}^{\prime}$ $=1.0: 0.9 ; 3 \mathrm{mmol}$, only $\mathbf{4} \mathbf{f}$ was observed). The same phenomenon was observed in the reaction of

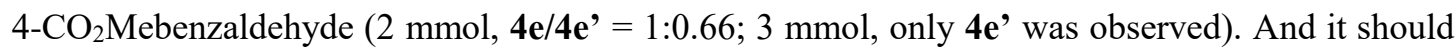
be noted that, no excess aromatic aldehydes were left in the reaction of $2 \mathrm{mmol}$ aldehyde reactions. Consequently, we thought the formation of $\mathbf{4} \mathbf{f}^{\prime}$ might derived from the shortage of aromatic aldehydes caused by themselves side reactions. However, the side reaction could not be identified by now.
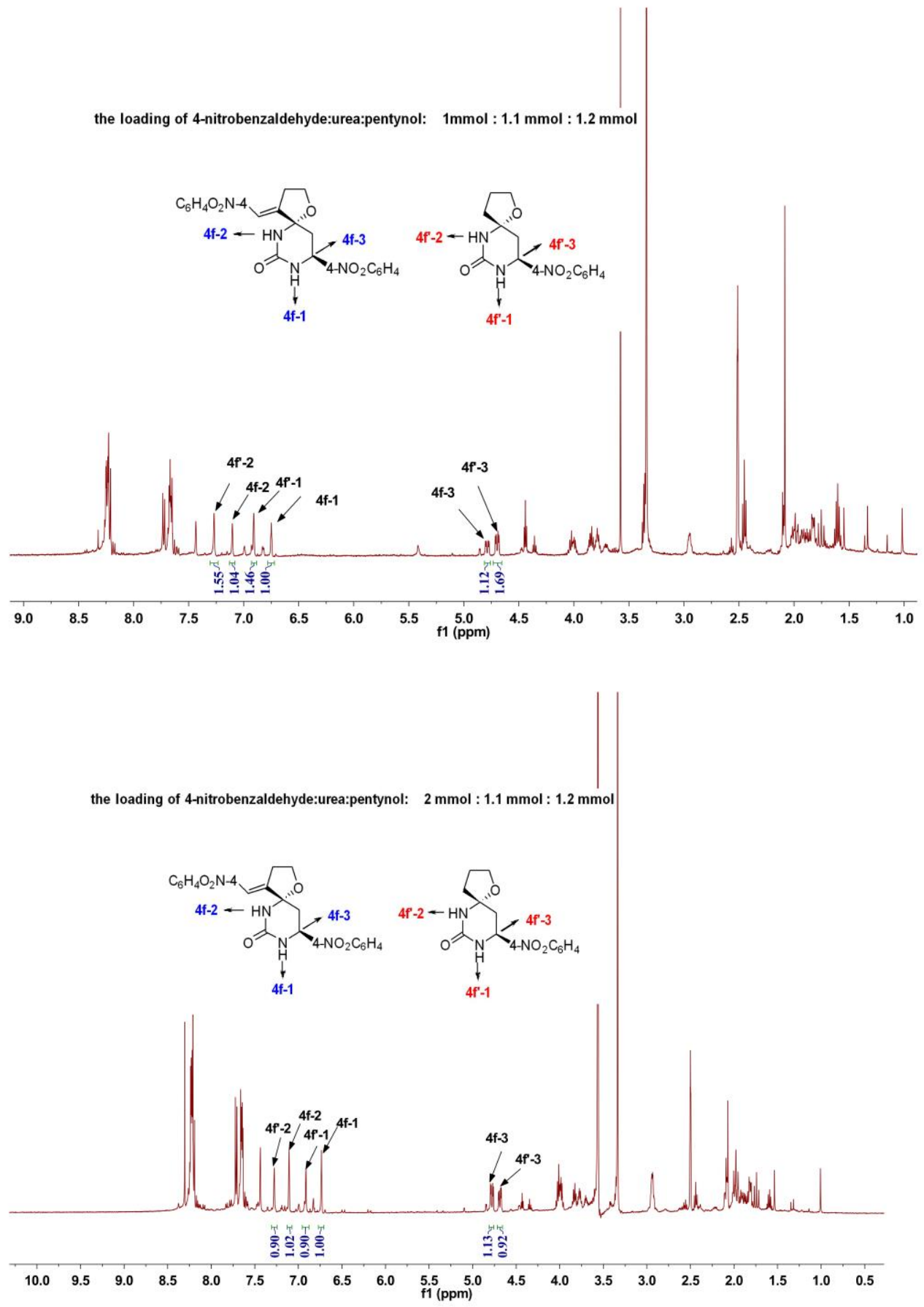


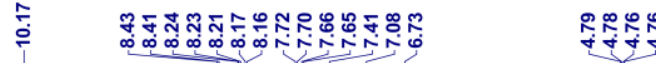

the loading of 4-nitrobenzaldehyde:urea:pentynol: $3 \mathrm{mmol}: 1.1 \mathrm{mmol}: 1.0 \mathrm{mmo}$
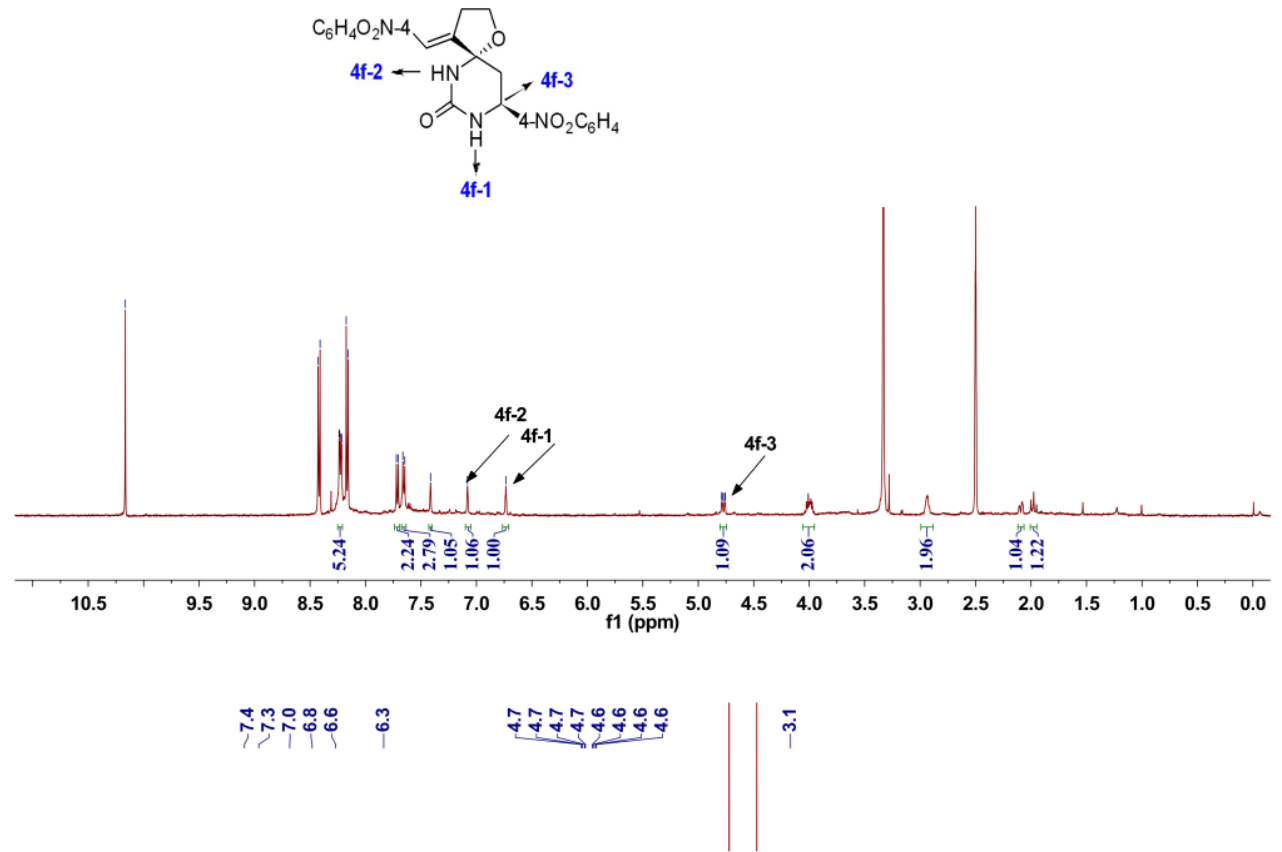

the loading of 4- $\mathrm{CO}_{2}$ Mebenzaldehyde:urea:pentynol: $2 \mathrm{mmol}: 1.1 \mathrm{mmol}: 1.2 \mathrm{mmol}$

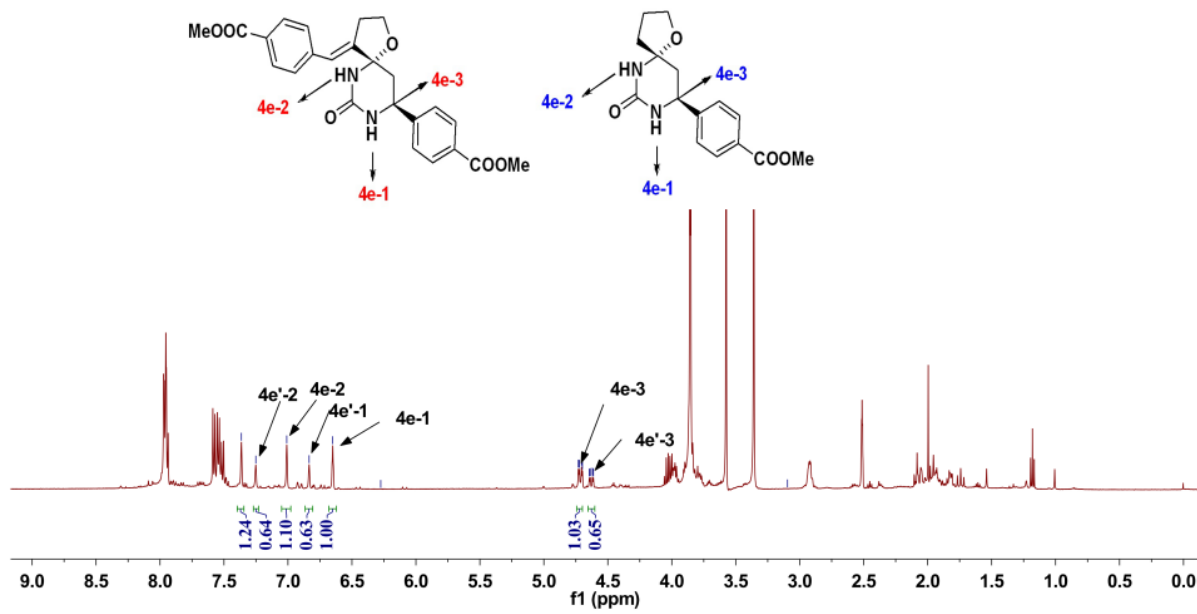


我施

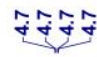

the loading of 4- $\mathrm{CO}_{2}$ Mebenzaldehyde:urea:pentynol: $3 \mathrm{mmol}: 1.1 \mathrm{mmol}: 1.0 \mathrm{mmol}$
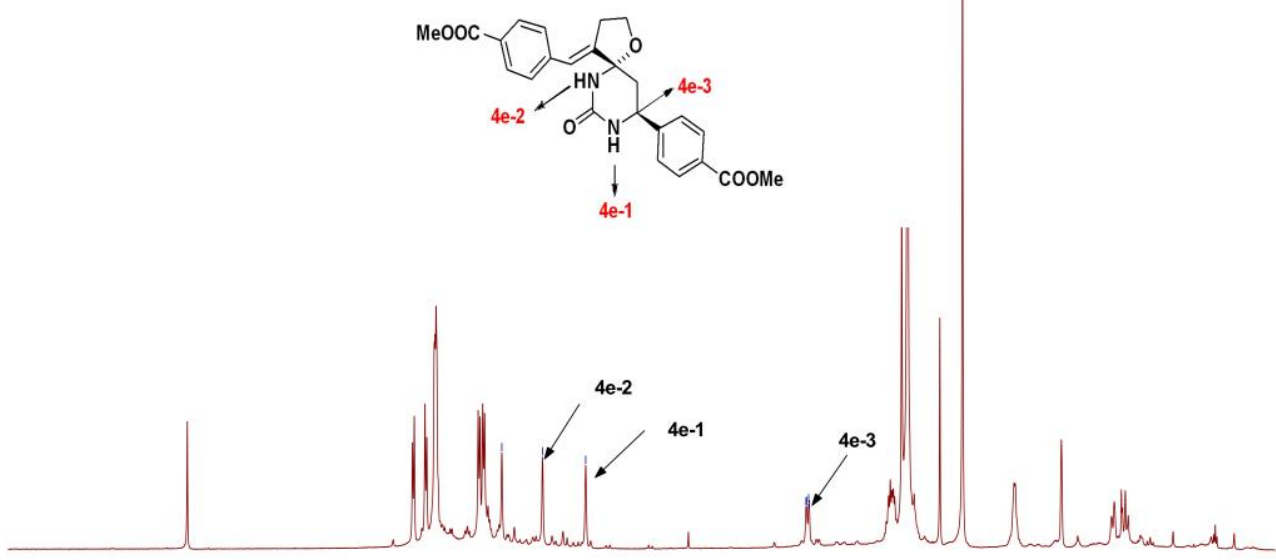

$\begin{array}{llllllllllllllllllllll}11.5 & 11.0 & 10.5 & 10.0 & 9.5 & 9.0 & 8.5 & 8.0 & 7.5 & 7.0 & 6.5 & 6.0 & 5.5 & 5.0 & 4.5 & 4.0 & 3.5 & 3.0 & 2.5 & 2.0 & 1.5 & 1.0\end{array}$ 
Based on the control experiments, a possible reaction pathway was proposed as shown in Scheme S3. As proposed in manuscript, rapid isomerization of exo-cyclic double bond gave endo-cyclic enol ether $\mathbf{D}$ in the presence of palladium catalyst. At this stage, the second aromatic aldehyde accepted the nucleophilic attack of enol ether under the activation of metal, giving oxonium intermediate E. Intramolecular cyclization followed by protonation and dehydration delivered the desired product 4 and regenerated palladium catalyst. In case of electron-deficient aromatic aldehyde, owing to the lack of sufficient aldehyde, part of enol ether $\mathbf{D}$ underwent intramolecular hydroamination to generate intermediate $\mathbf{F}$, which was converted to byproduct 4 , after protonation.

Scheme S3 The possible reaction pathway to compound 4 and 4'

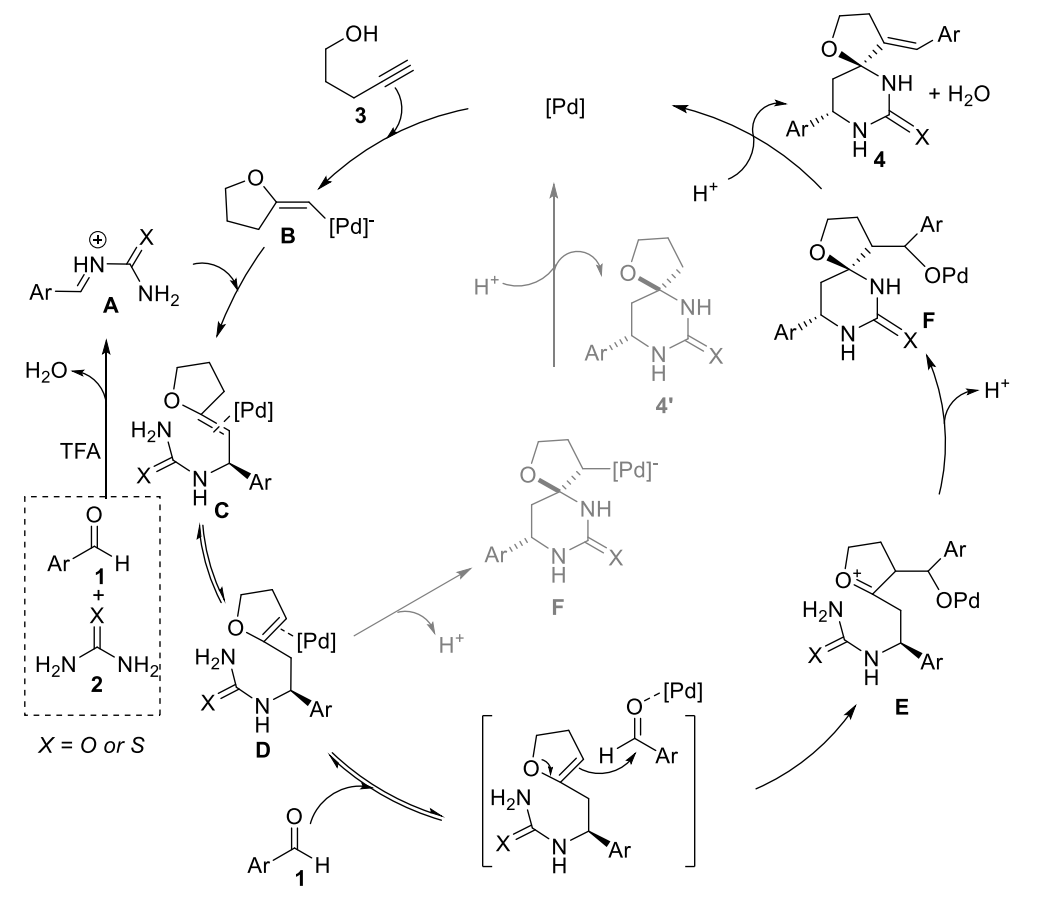


5. X-ray crystal structures of representative compounds

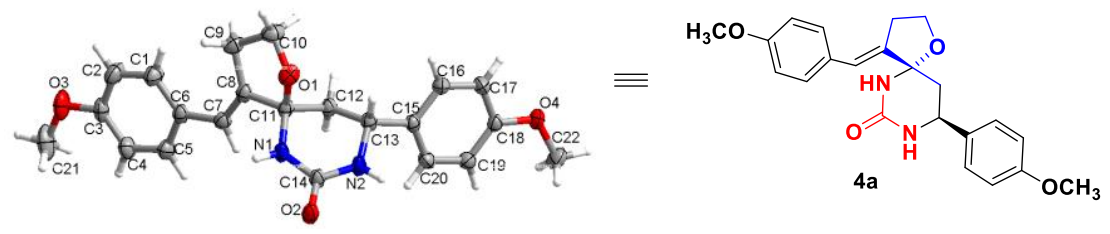

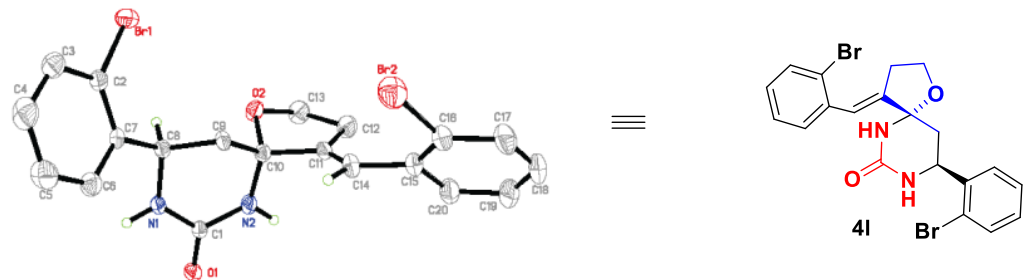

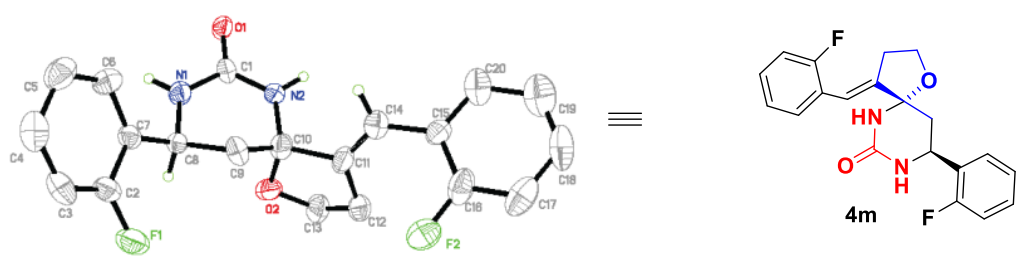

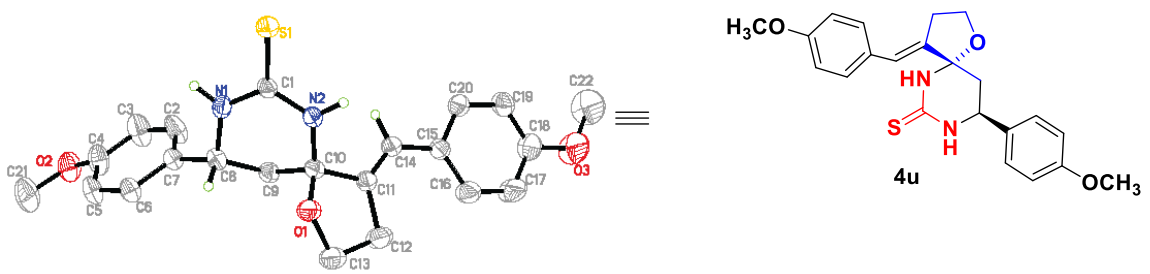

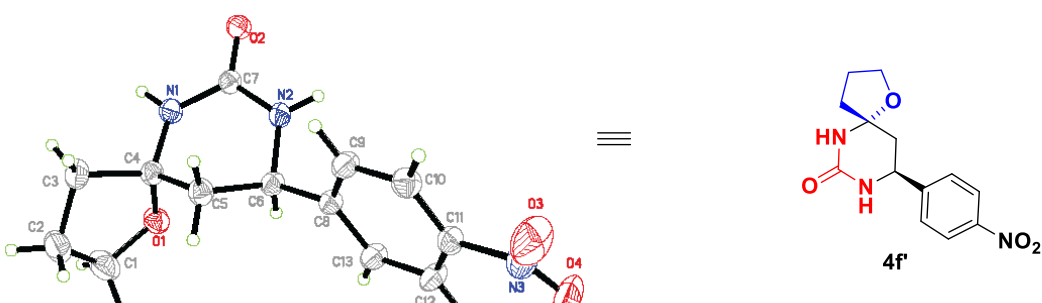




\section{Characterization data of new compounds}

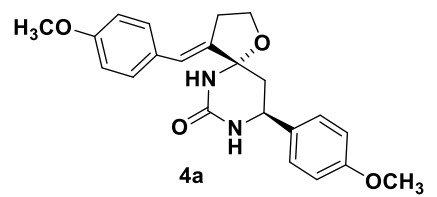

Following the general procedure, 4a was purified by $\mathrm{DCM} / \mathrm{MeOH}$ (50:1) and obtained as a white solid (330 $\mathrm{mg}, 87 \%$ yield); $\mathrm{Mp}=$ $130-131^{\circ} \mathrm{C} ;{ }^{1} \mathrm{H}$ NMR $\left(600 \mathrm{MHz}, \mathrm{DMSO}-d_{6}\right) \delta 7.32(\mathrm{dd}, J=11.8$, $8.8 \mathrm{~Hz}, 4 \mathrm{H}), 7.20$ (s, 1H), $6.92(\mathrm{dd}, J=17.4,8.7 \mathrm{~Hz}, 4 \mathrm{H}), 6.76(\mathrm{~s}, 1 \mathrm{H}), 6.47$ (s, 1H), 4.56 (dd, $J=$ 11.2, 4.6 Hz, 1H), 4.03-3.83 (m, 2H), $3.75(\mathrm{~s}, 3 \mathrm{H}), 3.73(\mathrm{~s}, 3 \mathrm{H}), 2.87-2.77(\mathrm{~m}, 2 \mathrm{H}), 1.94-1.88(\mathrm{~m}$, 2H) ppm; ${ }^{13} \mathrm{C}$ NMR (150 MHz, DMSO) $\delta 158.7,158.3,155.9,140.1,134.4,129.7,129.6,127.8$, $122.1,114.0,113.8,90.8,63.8,55.3,51.4,41.7,30.6 \mathrm{ppm}$;

HRMS: $\mathrm{C}_{22} \mathrm{H}_{25} \mathrm{~N}_{2} \mathrm{O}_{4}$ for $[\mathrm{M}+\mathrm{H}]^{+}$, calculated 381.1809, found 381.1803.

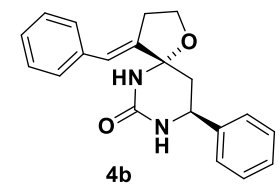

Following the general procedure, $\mathbf{4 b}$ was purified by $\mathrm{DCM} / \mathrm{MeOH}(50: 1)$ and obtained as a white solid $\left(254 \mathrm{mg}, 80 \%\right.$ yield); $\mathrm{Mp}=102-104^{\circ} \mathrm{C} ;{ }^{1} \mathrm{H}$ NMR (600 MHz, DMSO- $\left.d_{6}\right) \delta$ 7.42-7.25 (m, 11H), $6.91(\mathrm{~s}, 1 \mathrm{H}), 6.53(\mathrm{~s}, 1 \mathrm{H})$, $4.62(\mathrm{dd}, J=12.0 \mathrm{~Hz}, 3.6 \mathrm{~Hz}, 1 \mathrm{H}), 3.99-3.92(\mathrm{~m}, 2 \mathrm{H}), 2.90-2.81(\mathrm{~m}, 2 \mathrm{H}), 2.00(\mathrm{~d}, J=11.2 \mathrm{~Hz}$, $1 \mathrm{H}), 1.92(\mathrm{t}, J=12.6 \mathrm{~Hz}, 1 \mathrm{H}) \mathrm{ppm} ;{ }^{13} \mathrm{C} \mathrm{NMR}(150 \mathrm{MHz}, \mathrm{DMSO}) \delta 155.9,142.7,142.5,136.9$, $128.5,128.4,127.5,127.1,126.7,122.6,90.9,63.8,52.0,41.6,30.6 \mathrm{ppm}$;

HRMS: $\mathrm{C}_{20} \mathrm{H}_{21} \mathrm{~N}_{2} \mathrm{O}_{2}$ for $[\mathrm{M}+\mathrm{H}]^{+}$, calculated 321.1598, found 321.1607 .

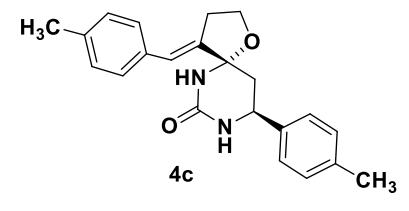

Following the general procedure, $\mathbf{4} \mathbf{c}$ was purified by $\mathrm{DCM} / \mathrm{MeOH}$ (50:1) and obtained as a white solid (296 $\mathrm{mg}, 85 \%$ yield); $\mathrm{Mp}=$ 120-122 ${ }^{\circ} \mathrm{C} ;{ }^{1} \mathrm{H}$ NMR $\left(600 \mathrm{MHz}, \mathrm{CDCl}_{3}\right) \delta: 7.29(\mathrm{~d}, J=6 \mathrm{~Hz}$, 2H),.7.23 (d, $J=6 \mathrm{~Hz}, 2 \mathrm{H}), 7.18(\mathrm{~d}, J=6 \mathrm{~Hz}, 4 \mathrm{H}), .6 .57$ (s, 1H), 5.49 (brs, $1 \mathrm{H}), 5.09$ (brs, $1 \mathrm{H}$ ), 4.78(dd, $J=6 \mathrm{~Hz}, 12 \mathrm{~Hz}, 1 \mathrm{H}), 4.04-4.02(\mathrm{~m}, 2 \mathrm{H}), 2.99-2.94(\mathrm{~m}, 1 \mathrm{H}), 2.92-2.87(\mathrm{~m}, 1 \mathrm{H}), 2.35(\mathrm{~d}$, $6 \mathrm{H}), 2.13(\mathrm{~d}, J=12 \mathrm{~Hz}, 1 \mathrm{H}), 1.96(\mathrm{t}, J=12 \mathrm{~Hz}, 1 \mathrm{H}) \mathrm{ppm} .{ }^{13} \mathrm{C} \mathrm{NMR}\left(150 \mathrm{MHz}, \mathrm{CDCl}_{3}\right) \delta 156.4$, 140.6, 138.2, 137.9, 137.5, 133.6, 129.6, 129.3, 128.4, 126.3, 124.4, 91.2, 64.7, 52.4, 42.2, 30.7, $21.2 \mathrm{ppm}$

HRMS: $\mathrm{C}_{22} \mathrm{H}_{25} \mathrm{~N}_{2} \mathrm{O}_{2}$ for $[\mathrm{M}+\mathrm{H}]^{+}$, calculated 349.1911, found 349.1910.

Following the general procedure, $\mathbf{4 d}$ was purified by $\mathrm{DCM} / \mathrm{MeOH}(30: 1)$ and obtained as a white 


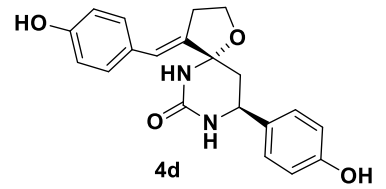

solid $(285 \mathrm{mg}, 81 \%$ yield $) ; \mathrm{Mp}=183-185 ;{ }^{1} \mathrm{H}$ NMR $(400 \mathrm{MHz}$, $\left.\mathrm{CD}_{3} \mathrm{OD}\right) \delta 7.24(\mathrm{dd}, J=8.6 \mathrm{~Hz}, 4.6 \mathrm{~Hz}, 4 \mathrm{H}), 6.79(\mathrm{dd}, J=7.6 \mathrm{~Hz}, 5.8$ $\mathrm{Hz}, 4 \mathrm{H}), 6.49(\mathrm{~s}, 1 \mathrm{H}), 4.65(\mathrm{dd}, J=12.2 \mathrm{~Hz}, 3.7 \mathrm{~Hz}, 1 \mathrm{H}), 4.09-3.95$ (m, 2H), 3.04-2.79 (m, 2H), $2.07(\mathrm{dd}, J=13.3 \mathrm{~Hz}, 3.8 \mathrm{~Hz}, 1 \mathrm{H}), 1.96(\mathrm{t}, J=13.3 \mathrm{~Hz}, 1 \mathrm{H}) \mathrm{ppm} ;{ }^{13} \mathrm{C}$ NMR (100 MHz, CD $\left.{ }_{3} \mathrm{OD}\right) \delta 159.2,158.4,158.0,139.9,133.5,131.1,129.8,128.9,125.0,116.5$, $116.3,92.3,65.8,53.2,43.1,31.7 \mathrm{ppm}$;

HRMS: $\mathrm{C}_{20} \mathrm{H}_{21} \mathrm{~N}_{2} \mathrm{O}_{4}$ for $[\mathrm{M}+\mathrm{H}]^{+}$, calculated 353.1501, found 353.1492 .

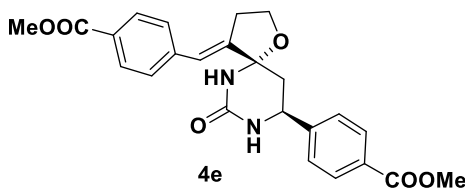

Following the general procedure, $4 \mathbf{e}$ was purified by $\mathrm{DCM} / \mathrm{MeOH}(50: 1)$ and obtained as a white solid (209 mg, 48\% yield $) ; \mathrm{Mp}=250-253^{\circ} \mathrm{C} ;{ }^{1} \mathrm{H} \mathrm{NMR}\left(600 \mathrm{MHz}, \mathrm{CDCl}_{3}\right) \delta 8.04(\mathrm{~d}$, $J=8.4 \mathrm{~Hz}, 2 \mathrm{H}), 8.02(\mathrm{~d}, J=8.4 \mathrm{~Hz}, 2 \mathrm{H}), 7.49(\mathrm{~d}, J=8.4 \mathrm{~Hz}, 2 \mathrm{H}), 7.38(\mathrm{~d}, J=8.4 \mathrm{~Hz}, 2 \mathrm{H}), 6.65$ (s, 1H), $5.71(\mathrm{~s}, 1 \mathrm{H}), 5.36(\mathrm{~s}, 1 \mathrm{H}), 4.89(\mathrm{dd}, J=12.2 \mathrm{~Hz}, 3.5 \mathrm{~Hz}, 1 \mathrm{H}), 4.08-4.05(\mathrm{~m}, 2 \mathrm{H}), 3.91(\mathrm{~s}$, $6 \mathrm{H}), 3.03-2.92(\mathrm{~m}, 2 \mathrm{H}), 2.20-2.17(\mathrm{~m}, 1 \mathrm{H}), 1.95(\mathrm{t}, J=12.2 \mathrm{~Hz}, 1 \mathrm{H}) \mathrm{ppm} ;{ }^{13} \mathrm{C}$ NMR $(150 \mathrm{MHz}$, $\left.\mathrm{CDCl}_{3}\right) \delta 166.7,166.6,156.0,146.0,144.2,140.6,130.3,130.2,129.8,129.1,128.4,126.4,124.2$, $91.1,64.3,52.6,52.2,42.5,30.8 \mathrm{ppm}$

HRMS: $\mathrm{C}_{24} \mathrm{H}_{25} \mathrm{~N}_{2} \mathrm{O}_{6}$ for $[\mathrm{M}+\mathrm{H}]^{+}$, calculated 437.1707, found 437.1711.

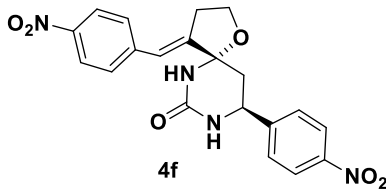

Following the general procedure, $4 \mathbf{a}$ was purified by $\mathrm{DCM} / \mathrm{MeOH}$ (50:1) and obtained as a light yellow solid (189 $\mathrm{mg}, 46 \%$ yield); Mp $5.5 \mathrm{~Hz}, 4 \mathrm{H}), 7.71(\mathrm{~d}, J=8.7 \mathrm{~Hz}, 2 \mathrm{H}), 7.66(\mathrm{~d}, J=8.9 \mathrm{~Hz}, 2 \mathrm{H}), 7.45(\mathrm{~s}, 1 \mathrm{H}), 7.12(\mathrm{~s}, 1 \mathrm{H}), 6.74(\mathrm{~s}$, 1H), $4.77(\mathrm{dd}, J=12.2 \mathrm{~Hz}, 3.7 \mathrm{~Hz}, 1 \mathrm{H}), 4.03-3.96(\mathrm{~m}, 2 \mathrm{H}), 2.97-2.89(\mathrm{~m}, 2 \mathrm{H}), 2.09(\mathrm{dd}, J=$ 13.1Hz, $3.7 \mathrm{~Hz}, 1 \mathrm{H}), 1.98(\mathrm{t}, J=12.6 \mathrm{~Hz}, 1 \mathrm{H}) \mathrm{ppm} ;{ }^{13} \mathrm{C} \mathrm{NMR}(150 \mathrm{MHz}, \mathrm{DMSO}) \delta 156.0,150.7$, $148.2,147.3,146.2,144.1,129.8,128.2,124.1,124.0,122.1,91.5,64.2,52.1,40.9,31.1 \mathrm{ppm}$; HRMS: $\mathrm{C}_{20} \mathrm{H}_{19} \mathrm{~N}_{4} \mathrm{O}_{6}$ for $[\mathrm{M}+\mathrm{H}]^{+}$, calculated 411.1299, found 411.1299.

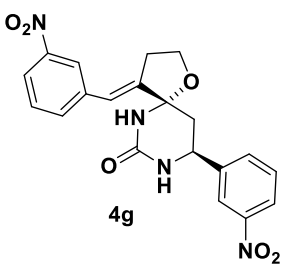

Following the general procedure, $\mathbf{4 g}$ was purified by $\mathrm{DCM} / \mathrm{MeOH}(50: 1)$ and obtained as a light yellow solid $(234 \mathrm{mg}, 57 \%$ yield $) ; \mathrm{Mp}=209-212^{\circ} \mathrm{C}$; 
${ }^{1} \mathrm{H}$ NMR $\left(600 \mathrm{MHz}, \mathrm{CDCl}_{3}\right) \delta 8.33(\mathrm{~s}, 1 \mathrm{H}), 8.22(\mathrm{~s}, 1 \mathrm{H}), 8.20(\mathrm{~d}, J=9.5 \mathrm{~Hz}, 1 \mathrm{H}), 8.13(\mathrm{~d}, J=7.3$ Hz, 1H), $7.78(\mathrm{~d}, J=7.8 \mathrm{~Hz}, 1 \mathrm{H}), 7.64(\mathrm{~d}, J=7.8 \mathrm{~Hz}, 1 \mathrm{H}), 7.59$ (t, $J=7.9 \mathrm{~Hz}, 1 \mathrm{H}), 7.55$ (t, $J=$ $8.0 \mathrm{~Hz}, 1 \mathrm{H}), 6.71(\mathrm{t}, J=2.5 \mathrm{~Hz}, 1 \mathrm{H}), 5.91(\mathrm{~s}, 1 \mathrm{H}), 5.72(\mathrm{~s}, 1 \mathrm{H}), 4.98(\mathrm{dd}, J=12.2 \mathrm{~Hz}, 3.5 \mathrm{~Hz}, 1 \mathrm{H})$, 4.12-4.09 (m, 2H), 3.04-2.98 (m, 2H), 2.25-2.22 (m, 1H), 2.01 (t, $J=12.7 \mathrm{~Hz}, 1 \mathrm{H}) \mathrm{ppm} ;{ }^{13} \mathrm{C}$ NMR (150 MHz, DMSO) $\delta 155.6,147.9,147.9,145.9,145.0,138.8,134.5,133.6,130.1,130.0,122.3$, 121.7, 121.3, 121.1, 90.7, 63.6, 51.8, 40.6, 30.5 ppm;

HRMS: $\mathrm{C}_{20} \mathrm{H}_{19} \mathrm{~N}_{4} \mathrm{O}_{6}$ for $[\mathrm{M}+\mathrm{H}]^{+}$, calculated 411.1299, found 411.1309.

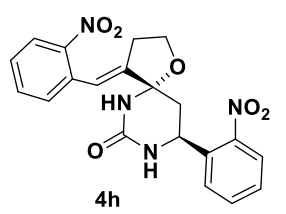

Following the general procedure, $4 \mathbf{h}$ was purified by $\mathrm{DCM} / \mathrm{MeOH}(50: 1)$ and obtained as a light yellow solid $(221 \mathrm{mg}, 54 \%$ yield $) ; \mathrm{Mp}=205-207^{\circ} \mathrm{C}$; ${ }^{1} \mathrm{H}$ NMR (400 MHz, $\left.\mathrm{CDCl}_{3}\right) \delta 7.99(\mathrm{dd}, J=8.2,1.1 \mathrm{~Hz}, 1 \mathrm{H}), 7.93-7.86(\mathrm{~m}$, 2H), $7.68(\mathrm{t}, J=7.7 \mathrm{~Hz}, 1 \mathrm{H}), 7.61(\mathrm{t}, J=7.6 \mathrm{~Hz}, 1 \mathrm{H}), 7.50-7.43(\mathrm{~m}, 2 \mathrm{H}), 7.41(\mathrm{~d}, J=7.3 \mathrm{~Hz}, 1 \mathrm{H})$, 6.89 (s, 1H), $6.50(\mathrm{~s}, 1 \mathrm{H}), 5.98(\mathrm{~s}, 1 \mathrm{H}), 5.30(\mathrm{dd}, J=11.8 \mathrm{~Hz}, 3.4 \mathrm{~Hz}, 1 \mathrm{H}), 4.05$ (dd, $J=14.3 \mathrm{~Hz}$, $8.2 \mathrm{~Hz}, 1 \mathrm{H}), 3.97(\mathrm{dd}, J=14.5 \mathrm{~Hz}, 8.1 \mathrm{~Hz}, 1 \mathrm{H}), 2.88-2.76(\mathrm{~m}, 1 \mathrm{H}), 2.73-2.55(\mathrm{~m}, 2 \mathrm{H}), 1.91(\mathrm{t}, J=$ $12 \mathrm{~Hz}, 1 \mathrm{H}) \mathrm{ppm} ;{ }^{13} \mathrm{C} \mathrm{NMR}(100 \mathrm{MHz}, \mathrm{CDCl} 3) \delta 156.9,148.7,147.8,145.0,136.4,134.0,132.2$, 130.6, 128.8, 128.4, 124.7, 124.7,120.9, 90.5, 64.4, 48.1, 39.8, 29.8 ppm; HRMS: $\mathrm{C}_{20} \mathrm{H}_{19} \mathrm{~N}_{4} \mathrm{O}_{6}$ for $[\mathrm{M}+\mathrm{H}]^{+}$, calculated 411.1299, found 411.1301.

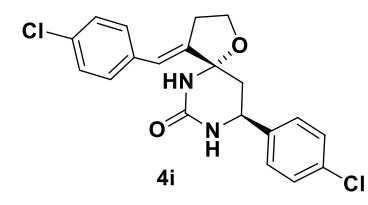

Following the general procedure, $4 \mathbf{i}$ was purified by $\mathrm{DCM} / \mathrm{MeOH}(50: 1)$ and obtained as a white solid (303 mg, 78\% yield); $\mathrm{Mp}=120-122^{\circ} \mathrm{C} ;{ }^{1} \mathrm{H}$ NMR (300 MHz, $\left.\mathrm{CDCl}_{3}\right)$ 8 7.46-7.29 (m, 6H), 7.26-7.21 (m, 2H), 6.57 (s, 1H), $6.00(\mathrm{~s}, 1 \mathrm{H}), 5.64(\mathrm{~s}, 1 \mathrm{H}), 4.80(\mathrm{dd}, J=12.2 \mathrm{~Hz}, 3.4 \mathrm{~Hz}, 1 \mathrm{H}), 4.09-4.04(\mathrm{~m}, 2 \mathrm{H})$, 3.01-2.86 (m, 2H), $2.15(\mathrm{~d}, J=12.6 \mathrm{~Hz}, 1 \mathrm{H}), 1.93(\mathrm{t}, J=12.6 \mathrm{~Hz}, 1 \mathrm{H}) \mathrm{ppm} ;{ }^{13} \mathrm{C} \mathrm{NMR}(75 \mathrm{MHz}$, $\left.\mathrm{CDCl}_{3}\right) \delta 155.5,142.6,140.0,135.0,133.8,133.2,129.8,129.1,128.7,127.8,123.4,91.1,64.7$, 52.4, 42.0, $30.7 \mathrm{ppm}$

HRMS: $\mathrm{C}_{20} \mathrm{H}_{19} \mathrm{Cl}_{2} \mathrm{~N}_{2} \mathrm{O}_{2}$ for $[\mathrm{M}+\mathrm{H}]^{+}$, calculated 389.0818 , found 389.0813 .

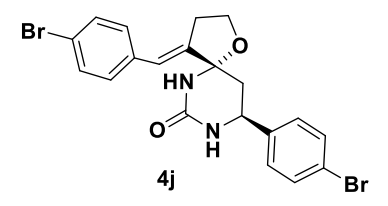

Following the general procedure, $\mathbf{4} \mathbf{j}$ was purified by $\mathrm{DCM} / \mathrm{MeOH}(50: 1)$ and obtained as a white solid (390 $\mathrm{mg}, 82 \%$ yield); $\mathrm{Mp}=135-138^{\circ} \mathrm{C}$; 
${ }^{1} \mathrm{H} \mathrm{NMR}\left(600 \mathrm{MHz}, \mathrm{CDCl}_{3}\right) \delta$ 7.51-7.47 (m, 4H), $7.28(\mathrm{~d}, J=8.4 \mathrm{~Hz}, 2 \mathrm{H}), 7.18(\mathrm{~d}, J=8.5 \mathrm{~Hz}$, 2H), $6.53(\mathrm{~s}, 1 \mathrm{H}), 5.69(\mathrm{~s}, 1 \mathrm{H}), 5.32(\mathrm{~s}, 1 \mathrm{H}), 4.78(\mathrm{dd}, J=12.2 \mathrm{~Hz}, 3.5 \mathrm{~Hz}, 1 \mathrm{H}), 4.08-3.99(\mathrm{~m}, 2 \mathrm{H})$, 2.99-2.82 (m, 2H), $2.16-2.09(\mathrm{~m}, 1 \mathrm{H}), 1.92(\mathrm{~d}, J=12.7 \mathrm{~Hz}, 1 \mathrm{H}) \mathrm{ppm} ;{ }^{13} \mathrm{C}$ NMR $(150 \mathrm{MHz}$, $\left.\mathrm{CDCl}_{3}\right) \delta 156.3,142.4,140.1,135.2,132.1,131.7,130.0,128.1,123.7,122.1,121.6,91.1,64.8$, 52.2, 42.0, 30.7 ppm;

HRMS: $\mathrm{C}_{20} \mathrm{H}_{19} \mathrm{Br}_{2} \mathrm{~N}_{2} \mathrm{O}_{2}$ for $[\mathrm{M}+\mathrm{H}]^{+}$, calculated 476.9808, found 476.9791 .

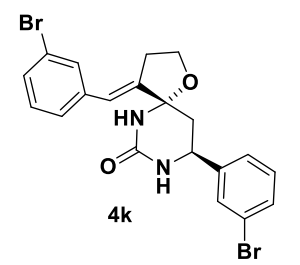

Following the general procedure, $\mathbf{4 k}$ was purified by $\mathrm{DCM} / \mathrm{MeOH}(50: 1)$ and obtained as a white solid (380 mg, $80 \%$ yield); $\mathrm{Mp}=117-119{ }^{\circ} \mathrm{C} ;{ }^{1} \mathrm{H}$ NMR (600 MHz, DMSO) $\delta 7.62(\mathrm{~s}, 1 \mathrm{H}), 7.56(\mathrm{~s}, 1 \mathrm{H}), 7.48(\mathrm{~d}, J=8.8 \mathrm{~Hz}$, 1H), $7.44(\mathrm{~d}, J=7.8 \mathrm{~Hz}, 1 \mathrm{H}), 7.42-7.38(\mathrm{~m}, 2 \mathrm{H}), 7.33$ (dd, $J=18.0 \mathrm{~Hz}, 7.2$ Hz, 3H), 6.99 (s, 1H), 6.56 (s, 1H), 4.61 (dd, $J=12.0 \mathrm{~Hz}, 3.9 \mathrm{~Hz}, 1 \mathrm{H}), 3.99-3.91$ (m, 2H), 2.89-2.82 (m, 2H), $2.01(\mathrm{dd}, J=12.6 \mathrm{~Hz}, 3.8 \mathrm{~Hz}, 1 \mathrm{H}), 1.94(\mathrm{t}, J=12.6 \mathrm{~Hz}, 1 \mathrm{H}) \mathrm{ppm} ;{ }^{13} \mathrm{C} \mathrm{NMR}$ (150 MHz, DMSO) $\delta 155.6,145.3,144.5,139.3,130.8,130.6,130.2,129.7,129.4,127.1,125.9$, $121.8,121.8,121.5,90.9,63.7,51.5,40.9,30.4 \mathrm{ppm}$;

HRMS: $\mathrm{C}_{20} \mathrm{H}_{19} \mathrm{Br}_{2} \mathrm{~N}_{2} \mathrm{O}_{2}$ for $[\mathrm{M}+\mathrm{H}]^{+}$, calculated 476.9808, found 476.9806 .

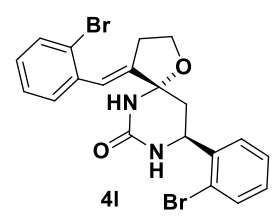

Following the general procedure, $\mathbf{4} \mathbf{l}$ was purified by $\mathrm{DCM} / \mathrm{MeOH}(50: 1)$ and obtained as a white solid (346 mg, 73\% yield); $\mathrm{Mp}=118-121^{\circ} \mathrm{C} ;{ }^{1} \mathrm{H}$ NMR $\left(600 \mathrm{MHz}, \mathrm{DMSO}-d_{6}\right) \delta$ 7.64-7.58 (m, 3H), 7.50-7.38 (m, 4H), $7.22(\mathrm{dt}, J=$ $15.3 \mathrm{~Hz}, 7.1 \mathrm{~Hz}, 2 \mathrm{H}), 7.07(\mathrm{~s}, 1 \mathrm{H}), 6.60(\mathrm{~s}, 1 \mathrm{H}), 5.03(\mathrm{~d}, J=11.8 \mathrm{~Hz}, 1 \mathrm{H})$, 3.99 (q, $J=7.4 \mathrm{~Hz}, 1 \mathrm{H}), 3.88$ (dd, $J=13.2 \mathrm{~Hz}, 8.0 \mathrm{~Hz}, 1 \mathrm{H}), 2.84-2.71(\mathrm{~m}, 2 \mathrm{H}), 2.19$ (d, $J=12.5$ $\mathrm{Hz}, 1 \mathrm{H}), 1.72(\mathrm{t}, J=12.5 \mathrm{~Hz}, 1 \mathrm{H}) ;{ }^{13} \mathrm{C}$ NMR $(150 \mathrm{MHz}, \mathrm{DMSO}) \delta 156.1,145.3,140.8,136.2$, 132.6, 132.6, 129.5, 129.4, 129.1, 128.3, 128.2, 127.7, 123.6, 121.9, 121.5, 90.4, 66.4, 61.9, 51.2, $30.1 \mathrm{ppm} ;$

HRMS: $\mathrm{C}_{20} \mathrm{H}_{19} \mathrm{Br}_{2} \mathrm{~N}_{2} \mathrm{O}_{2}$ for $[\mathrm{M}+\mathrm{H}]^{+}$, calculated 476.9808 , found 476.9807 .

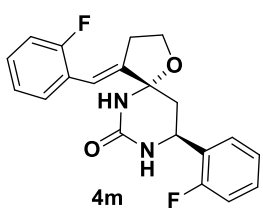

Following the general procedure, $\mathbf{4 m}$ was purified by $\mathrm{DCM} / \mathrm{MeOH}(50: 1)$ and obtained as a white solid (292 mg, 82\% yield); $\mathrm{Mp}=200-202{ }^{\circ} \mathrm{C} ;{ }^{1} \mathrm{H}$ NMR 
$\left(600 \mathrm{MHz}, \mathrm{CDCl}_{3}\right) \delta{ }^{1} \mathrm{H} \mathrm{NMR}\left(600 \mathrm{MHz}, \mathrm{CDCl}_{3}\right) \delta 7.54(\mathrm{t}, J=7.2 \mathrm{~Hz}, 1 \mathrm{H}), 7.34(\mathrm{t}, J=7.2 \mathrm{~Hz}$, 1H), 7.31-7.26 (m, 1H), 7.25-7.21 (m, 1H), $7.18(\mathrm{t}, J=7.4 \mathrm{~Hz}, 1 \mathrm{H}), 7.12(\mathrm{t}, J=7.4 \mathrm{~Hz}, 1 \mathrm{H})$, 7.08-7.01 (m, 2H), $6.73(\mathrm{~s}, 1 \mathrm{H}), 5.98(\mathrm{~s}, 1 \mathrm{H}), 5.62(\mathrm{~s}, 1 \mathrm{H}), 5.19(\mathrm{dd}, J=12.1,3.1 \mathrm{~Hz}, 1 \mathrm{H})$, 4.10-3.94 (m, 2H), 2.97-2.76 (m, 2H), 2.27 (d, $J=14.1 \mathrm{~Hz}, 1 \mathrm{H}), 1.95$ (t, $J=12.7 \mathrm{~Hz}, 1 \mathrm{H}) \mathrm{ppm}$; ${ }^{13} \mathrm{C}$ NMR $\left(150 \mathrm{MHz}, \mathrm{CDCl}_{3}\right) \delta 160.9,156.5,129.5,129.3,129.0,128.2,127.2,124.8,124.2$, 124.0, 91.0, 64.7, 45.9, 39.7, 31.0 ppm;

HRMS: $\mathrm{C}_{20} \mathrm{H}_{19} \mathrm{~F}_{2} \mathrm{~N}_{2} \mathrm{O}_{2}$ for $[\mathrm{M}+\mathrm{H}]^{+}$, calculated 357.1409, found 357.1402.

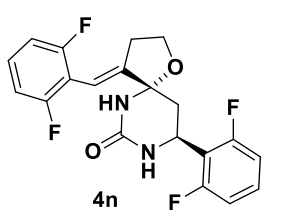

Following the general procedure, $4 \mathbf{n}$ was purified by $\mathrm{DCM} / \mathrm{MeOH}(50: 1)$ and obtained as a cream color solid $(318 \mathrm{mg}, 77 \%$ yield $) ; \mathrm{Mp}=187-189^{\circ} \mathrm{C} ;{ }^{1} \mathrm{H}$ NMR (600 MHz, DMSO) $\delta 7.41(\mathrm{dd}, J=16.6 \mathrm{~Hz}, 9.8 \mathrm{~Hz}, 3 \mathrm{H}), 7.16-7.08(\mathrm{~m}$, 4H), $7.06(\mathrm{~s}, 1 \mathrm{H}), 6.36(\mathrm{~s}, 1 \mathrm{H}), 5.03(\mathrm{dd}, J=12.5 \mathrm{~Hz}, 3.5 \mathrm{~Hz}, 1 \mathrm{H}), 3.88(\mathrm{dd}, J=11.2 \mathrm{~Hz}, 6.2 \mathrm{~Hz}$, 2H), 2.66-2.54 (m, 1H), 2.51-2.43 (m, 2H), $2.28(\mathrm{t}, J=12.6 \mathrm{~Hz}, 1 \mathrm{H}), 2.02(\mathrm{~d}, J=10.9 \mathrm{~Hz}, 1 \mathrm{H})$ ppm; ${ }^{13} \mathrm{C}$ NMR (150 MHz, DMSO) $\delta 161.8,160.3,158.6,155.4,149.4,129.8,116.4,113.4,112.1$, 111.7, 90.3, 63.8, 55.1, 42.1, 37.2, 30.9 ppm;

HRMS: $\mathrm{C}_{20} \mathrm{H}_{17} \mathrm{~N}_{2} \mathrm{O}_{2} \mathrm{~F}_{4}$ for $[\mathrm{M}+\mathrm{H}]^{+}$, calculated 415.1040, found 415.1034

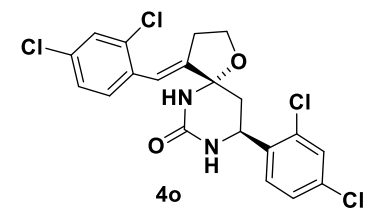

Following the general procedure, 40 was purified by $\mathrm{DCM} / \mathrm{MeOH}(50: 1)$ and obtained as a white solid ( $378 \mathrm{mg}, 83 \%$ yield); $\mathrm{Mp}=123-131^{\circ} \mathrm{C}$; ${ }^{1} \mathrm{H}$ NMR $\left(600 \mathrm{MHz}, \mathrm{CDCl}_{3}\right) \delta 7.58(\mathrm{~d}, J=8.4 \mathrm{~Hz}, 1 \mathrm{H}), 7.39(\mathrm{dd}, J=$ 10.5, 2.1 Hz, 2H), 7.33 (dd, $J=8.4,2.1 \mathrm{~Hz}, 1 \mathrm{H}), 7.28$ (t, $J=7.2 \mathrm{~Hz}, 1 \mathrm{H}), 7.24$ (dd, $J=8.4,2.0 \mathrm{~Hz}$, 1H), $6.72(\mathrm{~s}, 1 \mathrm{H}), 6.22(\mathrm{~s}, 1 \mathrm{H}), 6.04(\mathrm{~s}, 1 \mathrm{H}), 5.26(\mathrm{dd}, J=12.0,3.6 \mathrm{~Hz}, 1 \mathrm{H}), 4.07-4.01(\mathrm{~m}, 2 \mathrm{H})$, 2.85-2.81 (m, 2H), 2.37-2.33 (m, 1H), $1.75(\mathrm{t}, J=12.6 \mathrm{~Hz}, 1 \mathrm{H}) \mathrm{ppm} ;{ }^{13} \mathrm{C} \mathrm{NMR}\left(150 \mathrm{MHz}, \mathrm{CDCl}_{3}\right)$ $\delta 157.1,144.9,137.2,134.5,134.2,133.8,133.1,129.9,129.6,129.5,128.1,128.0,127.0,120.3$, 90.7, 64.7, 49.0, 39.0, 30.3 ppm.

HRMS: $\mathrm{C}_{20} \mathrm{H}_{17} \mathrm{Cl}_{4} \mathrm{~N}_{2} \mathrm{O}_{2}$ for $[\mathrm{M}+\mathrm{H}]^{+}$, calculated 457.0039, found 457.0039

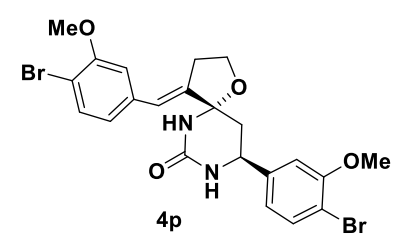

Following the general procedure, $4 \mathbf{p}$ was purified by $\mathrm{DCM} / \mathrm{MeOH}$ (50:1) and obtained as a white solid (445 $\mathrm{mg}, 83 \%$ yield); $\mathrm{Mp}=120$ 
$-122{ }^{\circ} \mathrm{C} ;{ }^{1} \mathrm{H}$ NMR $(600 \mathrm{MHz}, \mathrm{DMSO}) \delta 7.60(\mathrm{~d}, J=17.6 \mathrm{~Hz}, 2 \mathrm{H}), 7.37(\mathrm{dd}, J=15.1 \mathrm{~Hz}, 8.6 \mathrm{~Hz}$, 2H), $7.23(\mathrm{~s}, 1 \mathrm{H}), 7.12(\mathrm{~d}, J=8.7 \mathrm{~Hz}, 1 \mathrm{H}), 7.08(\mathrm{~d}, J=8.5 \mathrm{~Hz}, 1 \mathrm{H}), 6.85(\mathrm{~s}, 1 \mathrm{H}), 6.50(\mathrm{~s}, 1 \mathrm{H})$, 4.64-4.43 (m, 1H), 3.96-3.90 m, 2H), 3.85 (s, 3H), $3.83(\mathrm{~s}, 3 \mathrm{H}), 2.96-2.70(\mathrm{~m}, 2 \mathrm{H}), 1.99-1.90(\mathrm{~m}$, 2H) ppm; ${ }^{13} \mathrm{C}$ NMR (150 MHz, DMSO) $\delta 155.8,154.8,154.4,141.9,136.3,132.6,131.4,131.1$, 129.0, 127.5, 121.1, 112.7, 112.6, 110.9, 110.6, 90.9, 63.9, 56.4, 51.0, 41.2, 30.5 ppm; HRMS: $\mathrm{C}_{22} \mathrm{H}_{23} \mathrm{Br}_{2} \mathrm{~N}_{2} \mathrm{O}_{4}$ for $[\mathrm{M}+\mathrm{H}]^{+}$, calculated 537.0019, found 537.9986.

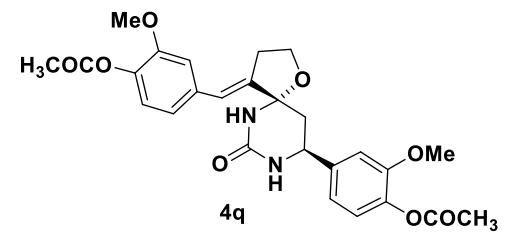
Following the general procedure, $\mathbf{4 q}$ was purified by $\mathrm{DCM} / \mathrm{MeOH}(50: 1)$ and obtained as a white solid (390 mg, 79\% yield); $\mathrm{Mp}=141-143{ }^{\circ} \mathrm{C} ;{ }^{1} \mathrm{H} \mathrm{NMR}\left(600 \mathrm{MHz}, \mathrm{CDCl}_{3}\right) \delta 7.02$ $(\mathrm{t}, J=6.6 \mathrm{~Hz}, 3 \mathrm{H}), 6.97(\mathrm{dd}, \mathrm{J}=8.1,1.6 \mathrm{~Hz}, 1 \mathrm{H}), 6.94-6.89(\mathrm{~m}, 2 \mathrm{H}), 6.58(\mathrm{~s}, 1 \mathrm{H}), 5.58(\mathrm{~s}, 1 \mathrm{H})$, $5.21(\mathrm{~s}, 1 \mathrm{H}), 4.81(\mathrm{dd}, \mathrm{J}=12.2 \mathrm{~Hz}, 3.4 \mathrm{~Hz}, 1 \mathrm{H}), 4.09-3.99(\mathrm{~m}, 2 \mathrm{H}), 3.85(\mathrm{~s}, 3 \mathrm{H}), 3.83$ (s, 3H), 2.94 (dt, $J=19.1,7.8 \mathrm{~Hz}, 2 \mathrm{H}), 2.31(\mathrm{~d}, \mathrm{~J}=2.7 \mathrm{~Hz}, 6 \mathrm{H}), 2.17(\mathrm{~d}, J=11.7 \mathrm{~Hz}, 1 \mathrm{H}), 1.95(\mathrm{t}, J=12.8 \mathrm{~Hz}$, 1H) ppm; ${ }^{13} \mathrm{C}$ NMR $\left(150 \mathrm{MHz}, \mathrm{CDCl}_{3}\right) \delta 169.1,156.1,151.4,150.9,141.7,139.9,139.4,139.0$, $135.2,124.2,123.1,122.8,120.6,118.5,112.7,110.2,91.1,66.9,64.6,56.0,55.7,52.5,42.2,30.5$ ppm;

HRMS: $\mathrm{C}_{26} \mathrm{H}_{29} \mathrm{~N}_{2} \mathrm{O}_{8}$ for $[\mathrm{M}+\mathrm{H}]^{+}$, calculated 497.1918, found 497.1917.

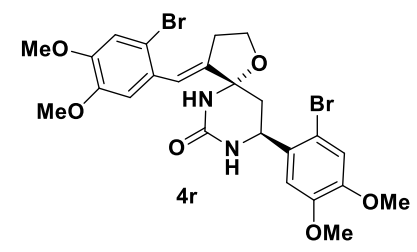

Following the general procedure, $4 \mathbf{r}$ was purified by $\mathrm{DCM} / \mathrm{MeOH}$ (50:1) and obtained as a white solid (511 $\mathrm{mg}, 86 \%$ yield); $\mathrm{Mp}=$ 129-130 ${ }^{\circ} \mathrm{C} ;{ }^{1} \mathrm{H} \mathrm{NMR}\left(600 \mathrm{MHz}, \mathrm{CDCl}_{3}\right) \delta 7.07(\mathrm{~s}, 1 \mathrm{H}), 6.98(\mathrm{~s}, 1 \mathrm{H})$, $6.94(\mathrm{~s}, 1 \mathrm{H}), 6.80(\mathrm{~s}, 1 \mathrm{H}), 6.66(\mathrm{~s}, 1 \mathrm{H}), 6.00(\mathrm{~s}, 1 \mathrm{H}), 5.82(\mathrm{~s}, 1 \mathrm{H})$, $5.14(\mathrm{dd}, J=11.9 \mathrm{~Hz}, 2.9 \mathrm{~Hz}, 1 \mathrm{H}), 4.00(\mathrm{dd}, J=24.5 \mathrm{~Hz}, 6.9 \mathrm{~Hz}, 2 \mathrm{H}), 3.88(\mathrm{~s}, 3 \mathrm{H}), 3.81(\mathrm{~d}, J=$ $5.4 \mathrm{~Hz}, 9 \mathrm{H}), 2.88-2.75(\mathrm{~m}, 2 \mathrm{H}), 2.33(\mathrm{~d}, J=11.5 \mathrm{~Hz}, 1 \mathrm{H}), 1.70(\mathrm{t}, J=12.6 \mathrm{~Hz}, 1 \mathrm{H}) \mathrm{ppm} ;{ }^{13} \mathrm{C}$ $\operatorname{NMR}\left(151 \mathrm{MHz}, \mathrm{CDCl}_{3}\right) \delta 156.4,149.1,147.6,142.7,131.8,127.8,123.6,115.5,114.9,112.1$, 111.6, 109.4, 90.7, 64.5, 56.2, 56.1, 56.1, 56.0, 51.4, 39.7, $30.3 \mathrm{ppm}$;

HRMS: $\mathrm{C}_{24} \mathrm{H}_{27} \mathrm{Br}_{2} \mathrm{~N}_{2} \mathrm{O}_{6}$ for $[\mathrm{M}+\mathrm{H}]^{+}$, calculated 599.0215, found 599.0212. 


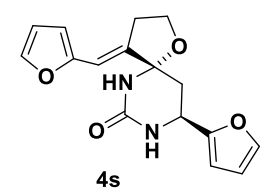

Following the general procedure, $4 \mathbf{s}$ was purified by $\mathrm{DCM} / \mathrm{MeOH}$ (50:1) and obtained as a white solid (218 mg, 73\% yield); $\mathrm{Mp}=$ 143-145 ${ }^{\circ} \mathrm{C} ;{ }^{1} \mathrm{H}$ NMR (600 MHz, DMSO- $\left.d_{6}\right) \delta 7.70(\mathrm{~s}, 1 \mathrm{H}), 7.61(\mathrm{~s}, 1 \mathrm{H}), 7.26$ (s, 1H), $6.85(\mathrm{~s}, 1 \mathrm{H}), 6.54(\mathrm{dd}, J=3.2 \mathrm{~Hz}, 1.8 \mathrm{~Hz}, 1 \mathrm{H}), 6.46(\mathrm{~d}, J=3.3 \mathrm{~Hz}, 1 \mathrm{H}), 6.42-6.39(\mathrm{~m}$, 3H), $4.65(\mathrm{dd}, J=11.8 \mathrm{~Hz}, 4.2 \mathrm{~Hz}, 1 \mathrm{H}), 3.96(\mathrm{dd}, J=15.5 \mathrm{~Hz}, 7.5 \mathrm{~Hz}, 1 \mathrm{H}), 3.90(\mathrm{td}, J=8.2 \mathrm{~Hz}$, $4.9 \mathrm{~Hz}, 1 \mathrm{H}), 2.94-2.87(\mathrm{~m}, 1 \mathrm{H}), 2.84-2.77(\mathrm{~m}, 1 \mathrm{H}), 2.09-1.99$ (m, 2H) ppm; ${ }^{13} \mathrm{C}$ NMR $(150 \mathrm{MHz}$, DMSO- $\left.d_{6}\right) \delta 156.9,156.6,143.2,130.6,129.1,128.7,126.8,125.7,121.1,120.7,117.1,111.4$, $111.2,91.4,64.1,55.5,46.0,31.2 \mathrm{ppm}$;

HRMS: $\mathrm{C}_{16} \mathrm{H}_{17} \mathrm{~N}_{2} \mathrm{O}_{4}$ for $[\mathrm{M}+\mathrm{H}]^{+}$, calculated 301.1183, found 301.1178 .

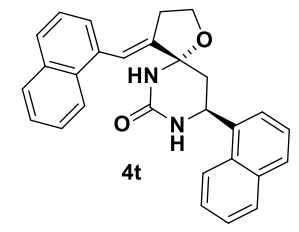

Following the general procedure, $\mathbf{4 t}$ was purified by $\mathrm{DCM} / \mathrm{MeOH}(50: 1)$ and obtained as a white solid (339mg, $81 \%$ yield); $\mathrm{Mp}=131-134{ }^{\circ} \mathrm{C} ; \delta{ }^{1} \mathrm{H}$ NMR $\left(400 \mathrm{MHz}, \mathrm{CDCl}_{3}\right) \delta 8.16(\mathrm{~d}, J=8.2 \mathrm{~Hz}, 1 \mathrm{H}), 7.93(\mathrm{t}, J=6.7 \mathrm{~Hz}, 2 \mathrm{H}), 7.85$ (d, $J=7.6 \mathrm{~Hz}, 2 \mathrm{H}), 7.79(\mathrm{~d}, J=7.9 \mathrm{~Hz}, 2 \mathrm{H}), 7.56-7.41(\mathrm{~m}, 6 \mathrm{H}), 7.37$ (d, $J=6.9 \mathrm{~Hz}, 1 \mathrm{H}), 7.25(\mathrm{~s}$, 1H), $5.82(\mathrm{~d}, J=11.7 \mathrm{~Hz}, 1 \mathrm{H}), 5.70(\mathrm{~s}, 1 \mathrm{H}), 5.21(\mathrm{~s}, 1 \mathrm{H}), 4.23-3.94(\mathrm{~m}, 2 \mathrm{H}), 2.98-2.73(\mathrm{~m}, 2 \mathrm{H})$, $2.58(\mathrm{~d}, J=15.8 \mathrm{~Hz}, 1 \mathrm{H}), 2.23(\mathrm{t}, J=13.2 \mathrm{~Hz}, 1 \mathrm{H}) ;{ }^{13} \mathrm{C} \mathrm{NMR}\left(150 \mathrm{MHz}, \mathrm{CDCl}_{3}\right) \delta 144.6,136.8$, 134.0, 133.6, 133.5, 131.5, 130.4, 129.1, 128.7, 128.6, 128.2, 126.7, 126.4, 126.0, 125.7, 125.2, $124.3,123.0,122.4,122.2,90.8,64.4,48.4,41.4,30.6$ ppm;

HRMS: $\mathrm{C}_{28} \mathrm{H}_{25} \mathrm{~N}_{2} \mathrm{O}_{2}$ for $[\mathrm{M}+\mathrm{H}]^{+}$, calculated 421.1916, found 421.1915 .

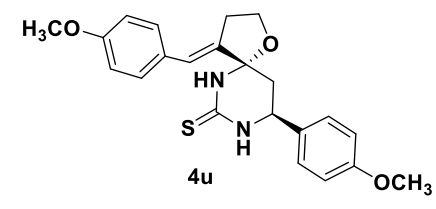

Following the general procedure, $\mathbf{4} \mathbf{j}$ was purified by $\mathrm{n}$-Hexane/EA (4:1) and obtained as a white solid (224 mg, 57\% yield); $\mathrm{Mp}=214$ $-216{ }^{\circ} \mathrm{C} ;{ }^{1} \mathrm{H}$ NMR $\left(400 \mathrm{MHz}, \mathrm{CDCl}_{3}\right) \delta 7.42(\mathrm{~s}, 1 \mathrm{H}), 7.27(\mathrm{dd}, J=$ 8.6, 2.2 Hz, 4H), $6.88(\mathrm{~d}, J=7.9 \mathrm{~Hz}, 4 \mathrm{H}), 6.79(\mathrm{~s}, 1 \mathrm{H}), 6.53(\mathrm{~s}, 1 \mathrm{H}), 4.72(\mathrm{dd}, J=12.3 \mathrm{~Hz}, 3.8 \mathrm{~Hz}$, 1H), 4.10-4.00 (m, 2H), $3.79(\mathrm{~d}, J=3.7 \mathrm{~Hz}, 6 \mathrm{H}), 2.90(\mathrm{dt}, J=18.7 \mathrm{~Hz}, 12.2 \mathrm{~Hz}, 2 \mathrm{H}), 2.12(\mathrm{~d}, J=$ $11.5 \mathrm{~Hz}, 1 \mathrm{H}), 2.04(\mathrm{~d}, J=13.7 \mathrm{~Hz}, 1 \mathrm{H}) \mathrm{ppm} ;{ }^{13} \mathrm{C} \mathrm{NMR}(100 \mathrm{MHz}, \mathrm{CDCl} 3) \delta 178.3,159.8,159.2$, 157.6, 138.2, 131.5, 130.2, 128.6, 127.8, 124.6, 114.4, 114.0, 90.2, 65.3, 55.4, 53.7, 40.4, 30.9 ppm;

HRMS: $\mathrm{C}_{22} \mathrm{H}_{25} \mathrm{~N}_{2} \mathrm{O}_{3} \mathrm{~S}$ for $[\mathrm{M}+\mathrm{H}]^{+}$, calculated 397.1586, found 397.1584. 


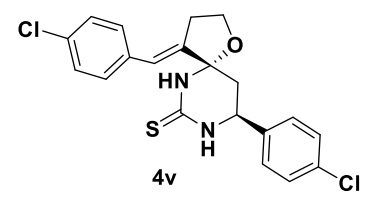

Following the general procedure, $4 \mathbf{v}$ was purified by $n$-Hexane/EA (4:1) and obtained as a white solid (180 mg, 45\% yield); $\mathrm{Mp}=216-217{ }^{\circ} \mathrm{C}$; ${ }^{1} \mathrm{H}$ NMR $\left(300 \mathrm{MHz}, \mathrm{DMSO}-d_{6}\right) \delta 8.86(\mathrm{~s}, 1 \mathrm{H}), 8.62(\mathrm{~s}, 1 \mathrm{H}), 7.44(\mathrm{~s}$, 8H), $6.60(\mathrm{~s}, 1 \mathrm{H}), 4.62(\mathrm{dd}, J=12.1 \mathrm{~Hz}, 4.0 \mathrm{~Hz}, 1 \mathrm{H}), 4.00(\mathrm{dd}, J=12.1 \mathrm{~Hz}, 7.4 \mathrm{~Hz}, 2 \mathrm{H}), 2.87$ (dt, $J=18.7 \mathrm{~Hz}, 12.1 \mathrm{~Hz}, 2 \mathrm{H}), 2.26-1.85(\mathrm{~m}, 2 \mathrm{H}) ;{ }^{13} \mathrm{C}$ NMR (100 MHz, DMSO- $\left.d_{6}\right) \delta 177.4,142.7$, 139.6, 135.8, 132.4, 131.6, 129.9, 129.0, 128.3, 121.7, 89.3, 64.6, 52.6, 30.8 ppm;

HRMS: $\mathrm{C}_{20} \mathrm{H}_{19} \mathrm{~N}_{2} \mathrm{OSCl}_{2}$ for $[\mathrm{M}+\mathrm{H}]^{+}$, calculated 405.0595, found 405.0583.

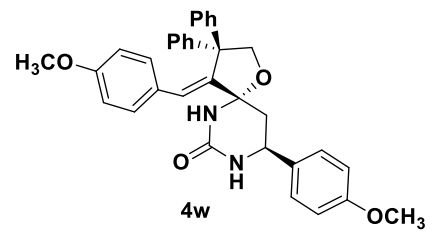

Following the general procedure, $\mathbf{4 w}$ was purified by $\mathrm{DCM} / \mathrm{MeOH}$ (50:1) and obtained as a white solid (328 $\mathrm{mg}, 62 \%$ yield); Mp = 195-197 ${ }^{\circ} \mathrm{C} ;{ }^{1} \mathrm{H} \mathrm{NMR}\left(500 \mathrm{MHz}, \mathrm{CDCl}_{3}\right) \delta 7.42(\mathrm{~d}, J=7.4 \mathrm{~Hz}, 2 \mathrm{H})$, 7.26-7.35 (m, 6H), 7.21 (d, $J=7.4 \mathrm{~Hz}, 2 \mathrm{H}), 7.07-7.19(\mathrm{~m}, 3 \mathrm{H}), 7.02$ $(\mathrm{s}, 1 \mathrm{H}), 6.90(\mathrm{~d}, J=8.7 \mathrm{~Hz}, 2 \mathrm{H}), 6.79(\mathrm{~d}, J=8.8 \mathrm{~Hz}, 2 \mathrm{H}), 6.40(\mathrm{~d}, J=8.8 \mathrm{~Hz}, 2 \mathrm{H}), 5.65(\mathrm{~s}, 1 \mathrm{H})$, $4.94(\mathrm{~s}, 1 \mathrm{H}), 4.71(\mathrm{dd}, J=9.8,6.0 \mathrm{~Hz}, 1 \mathrm{H}), 4.52(\mathrm{~d}, J=9.5 \mathrm{~Hz}, 1 \mathrm{H}), 4.46(\mathrm{~d}, J=9.5 \mathrm{~Hz}, 1 \mathrm{H}), 3.81$ $(\mathrm{s}, 3 \mathrm{H}), 3.64(\mathrm{~s}, 3 \mathrm{H}), 2.12-2.20(\mathrm{~m}, 2 \mathrm{H}) ;{ }^{13} \mathrm{C} \mathrm{NMR}\left(125 \mathrm{MHz}, \mathrm{CDCl}_{3}\right) \delta 159.7,159.1,156.4$, $131.1,129.5,129.3,128.6,128.2,127.9,127.4,127.0,114.4,112.8,93.6,81.5,61.5,55.5,55.2$, 52.3, 43.6 ppm;

HRMS: $\mathrm{C}_{34} \mathrm{H}_{32} \mathrm{~N}_{2} \mathrm{O}_{4}$ for $[\mathrm{M}+\mathrm{H}]^{+}$, calculated 533.2440, found 533.2444.

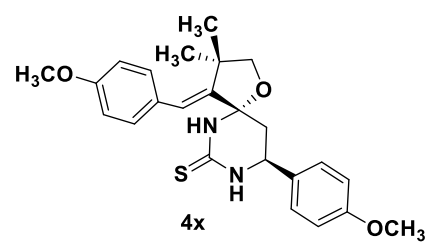

Following the general procedure, $\mathbf{4 x}$ was purified by $\mathrm{DCM} / \mathrm{MeOH}$ (50:1) and obtained as a light yellow solid with an inseparable unidentified by-product (302 mg, 67\% yield including unidentified unpurities); ${ }^{1} \mathrm{H}$ NMR (500 MHz, $\left.\mathrm{CDCl}_{3}\right) \delta 7.33(\mathrm{~d}, J=8.7 \mathrm{~Hz}, 2 \mathrm{H})$, $7.13(\mathrm{~d}, J=8.5 \mathrm{~Hz}, 2 \mathrm{H}), 6.91(\mathrm{~d}, J=8.7 \mathrm{~Hz}, 2 \mathrm{H}), 6.86(\mathrm{~d}, J=8.7 \mathrm{~Hz}, 2 \mathrm{H}), 6.68(\mathrm{~s}, 1 \mathrm{H}), 5.43(\mathrm{~s}$, 1H), $4.91(\mathrm{~s}, 1 \mathrm{H}), 4.79$ (dd, $J=12.3 \mathrm{~Hz}, 3.5 \mathrm{~Hz}, 1 \mathrm{H}), 3.81(\mathrm{~s}, 6 \mathrm{H}), 3.67$ (d, $J=8.7 \mathrm{~Hz}, 1 \mathrm{H}), 3.59$ $(\mathrm{d}, J=8.7 \mathrm{~Hz}, 1 \mathrm{H}), 2.25-2.18(\mathrm{~m}, 1 \mathrm{H}), 1.98(\mathrm{t}, J=12.8 \mathrm{~Hz}, 1 \mathrm{H}), 1.25(\mathrm{~s}, 3 \mathrm{H}), 1.03(\mathrm{~s}, 3 \mathrm{H})$;

HRMS: $\mathrm{C}_{24} \mathrm{H}_{28} \mathrm{~N}_{2} \mathrm{O}_{4}$ for $[\mathrm{M}+\mathrm{H}]^{+}$, calculated 409.2127, found 409.2132.

Following the general procedure, $4 \mathbf{y}$ was purified by DCM/MeOH (50:1) and obtained as a light yellow solid with an inseparable unidentified by-product $(335 \mathrm{mg}, 77 \%$ yield including S18 


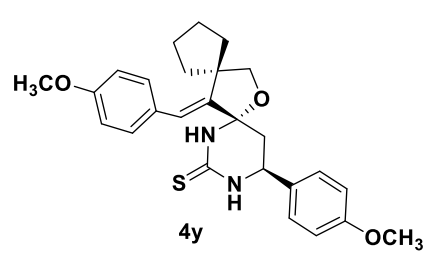

unidentified unpurities); ${ }^{1} \mathrm{H}$ NMR $\left(500 \mathrm{MHz}, \mathrm{CDCl}_{3}\right) \delta 7.33$ (d, $J=$ $8.7 \mathrm{~Hz}, 2 \mathrm{H}), 7.12(\mathrm{~d}, J=8.6 \mathrm{~Hz}, 2 \mathrm{H}), 6.90(\mathrm{~d}, J=8.7 \mathrm{~Hz}, 2 \mathrm{H}), 6.85$ (d, $J=8.7 \mathrm{~Hz}, 2 \mathrm{H}), 6.64(\mathrm{~s}, 1 \mathrm{H}), 5.53(\mathrm{~s}, 1 \mathrm{H}), 5.07(\mathrm{~s}, 1 \mathrm{H}), 4.77(\mathrm{dd}$, $J=12.3 \mathrm{~Hz}, 3.5 \mathrm{~Hz}, 1 \mathrm{H}), 3.80$ (s, 6H), 3.65-3.58 (m, 2H), 2.17 (ddd, $J=13.4 \mathrm{~Hz}, 7.6 \mathrm{~Hz}, 5.8 \mathrm{~Hz}, 1 \mathrm{H}), 1.95(\mathrm{t}, J=12.9 \mathrm{~Hz}, 1 \mathrm{H}), 1.77-1.59(\mathrm{~m}, 8 \mathrm{H})$;

HRMS: $\mathrm{C}_{26} \mathrm{H}_{31} \mathrm{~N}_{2} \mathrm{O}_{4}$ for $[\mathrm{M}+\mathrm{H}]^{+}$, calculated 435.2284 , found 435.2287 


\section{NMR spectra of new compounds}

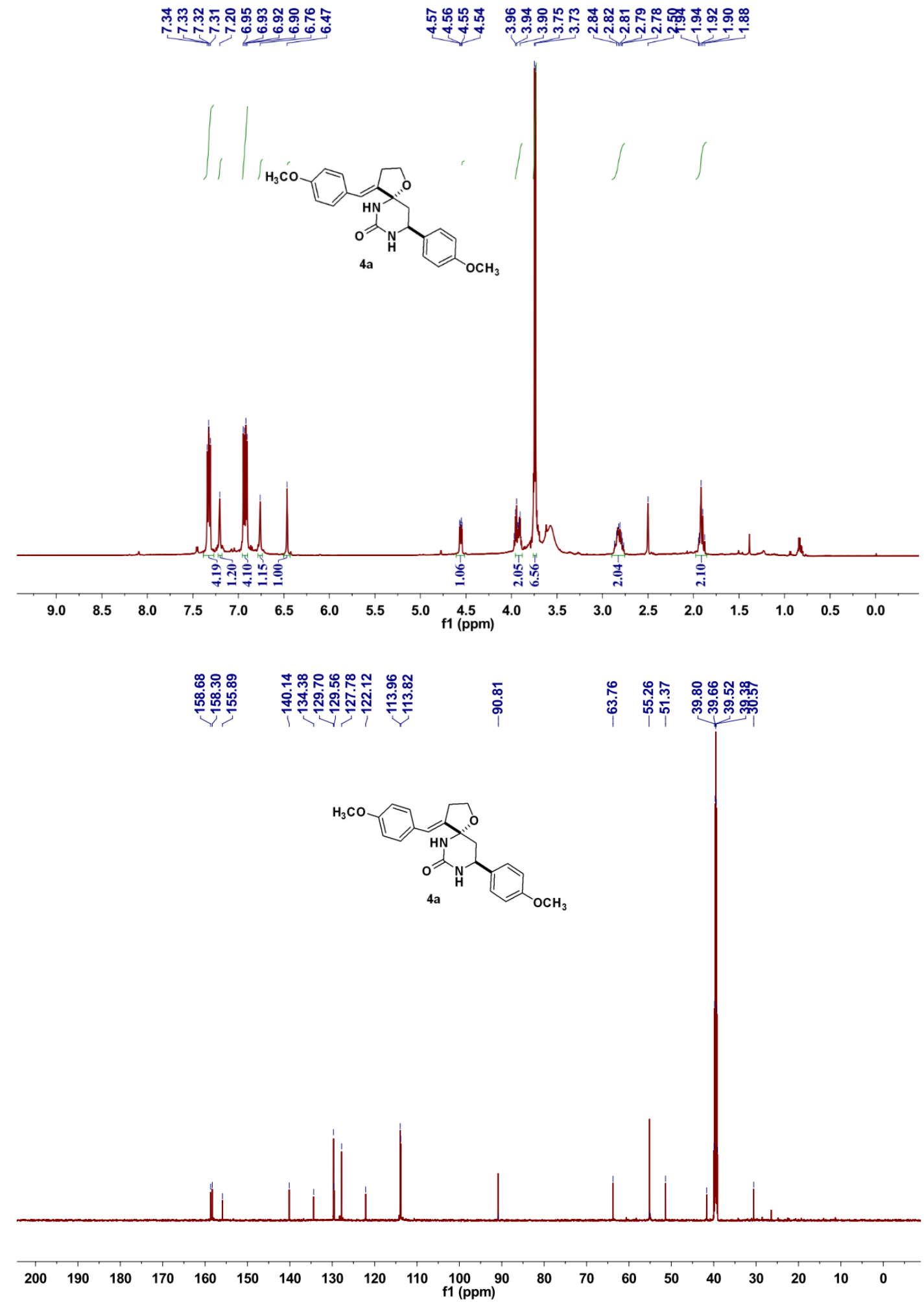



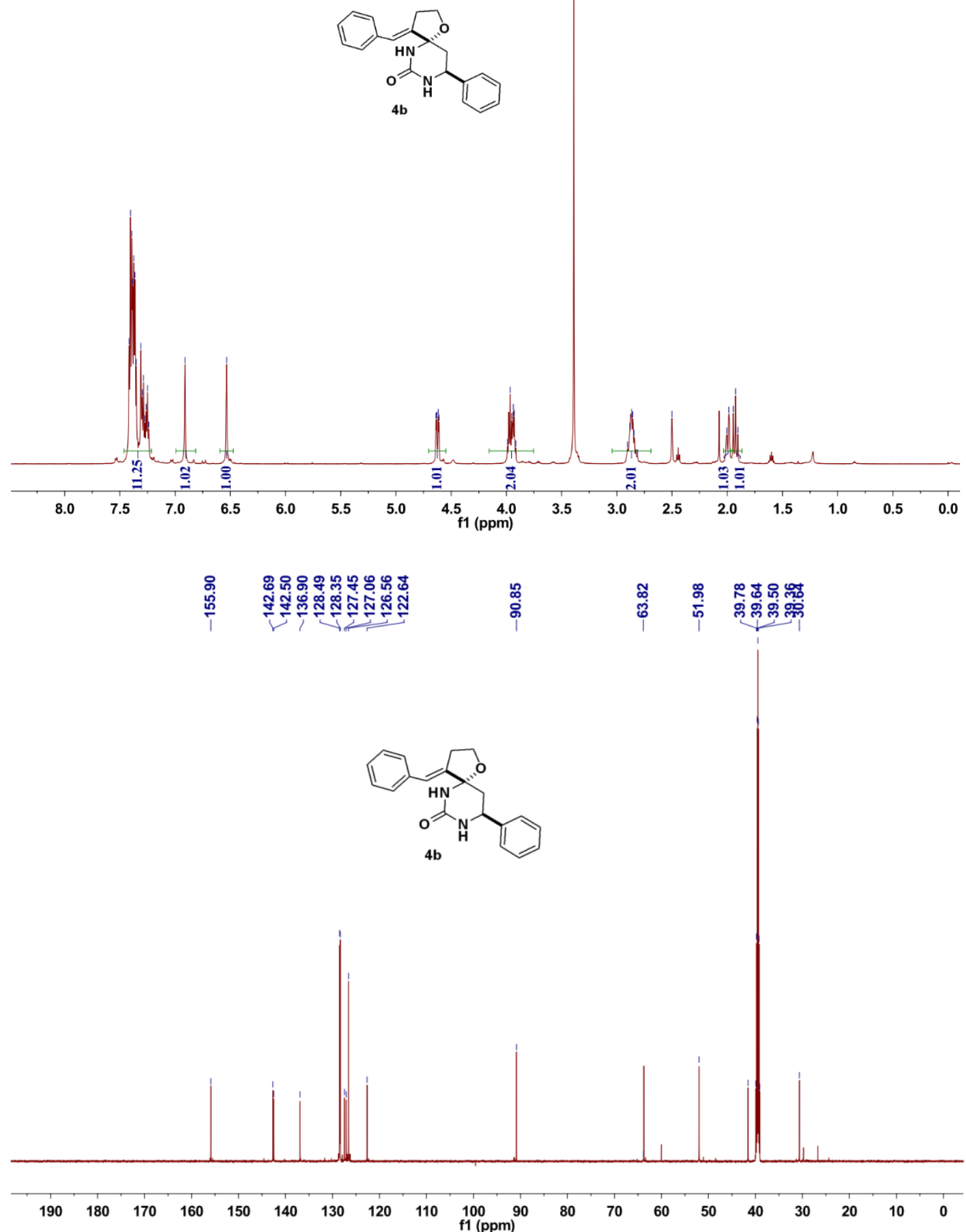

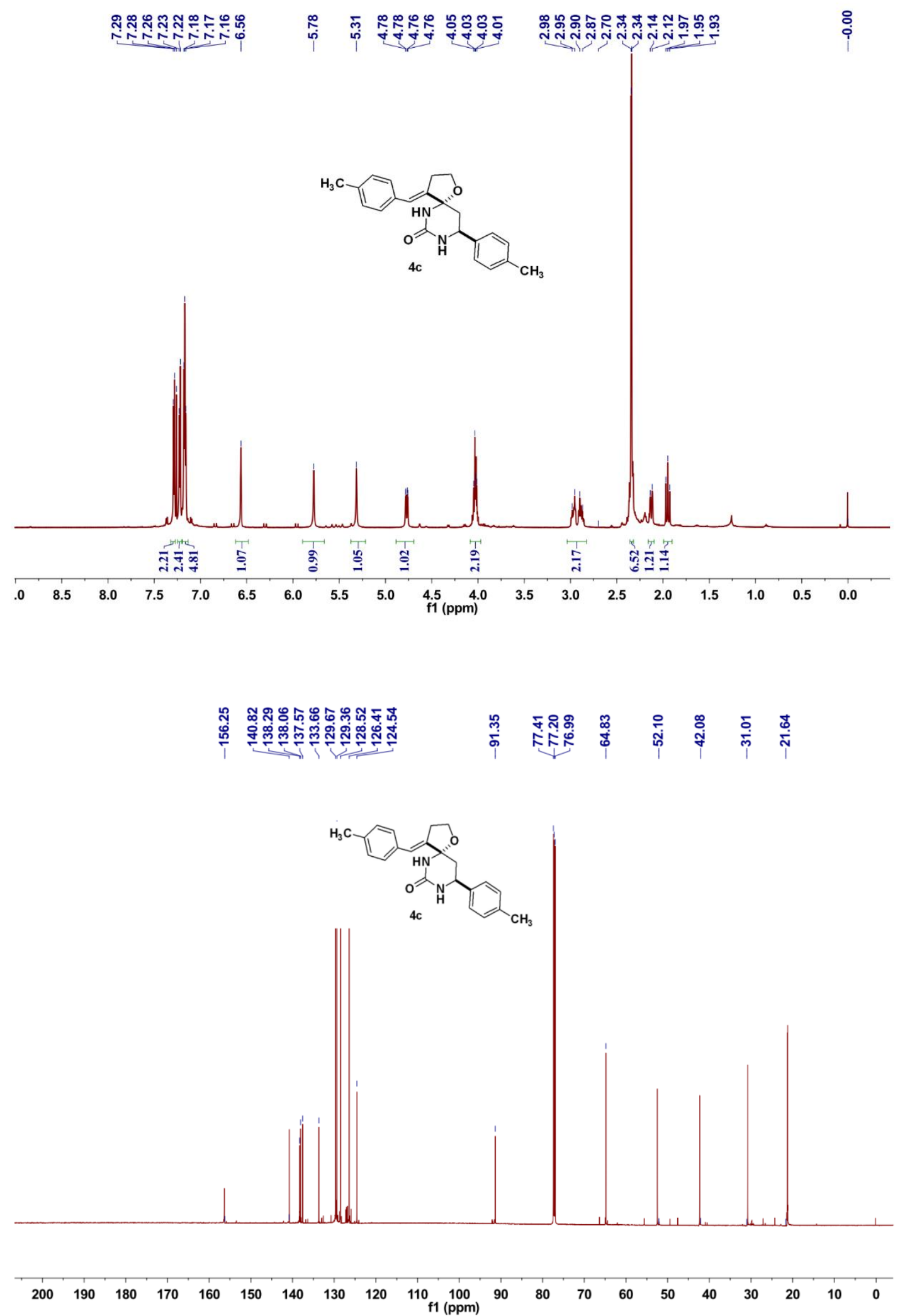


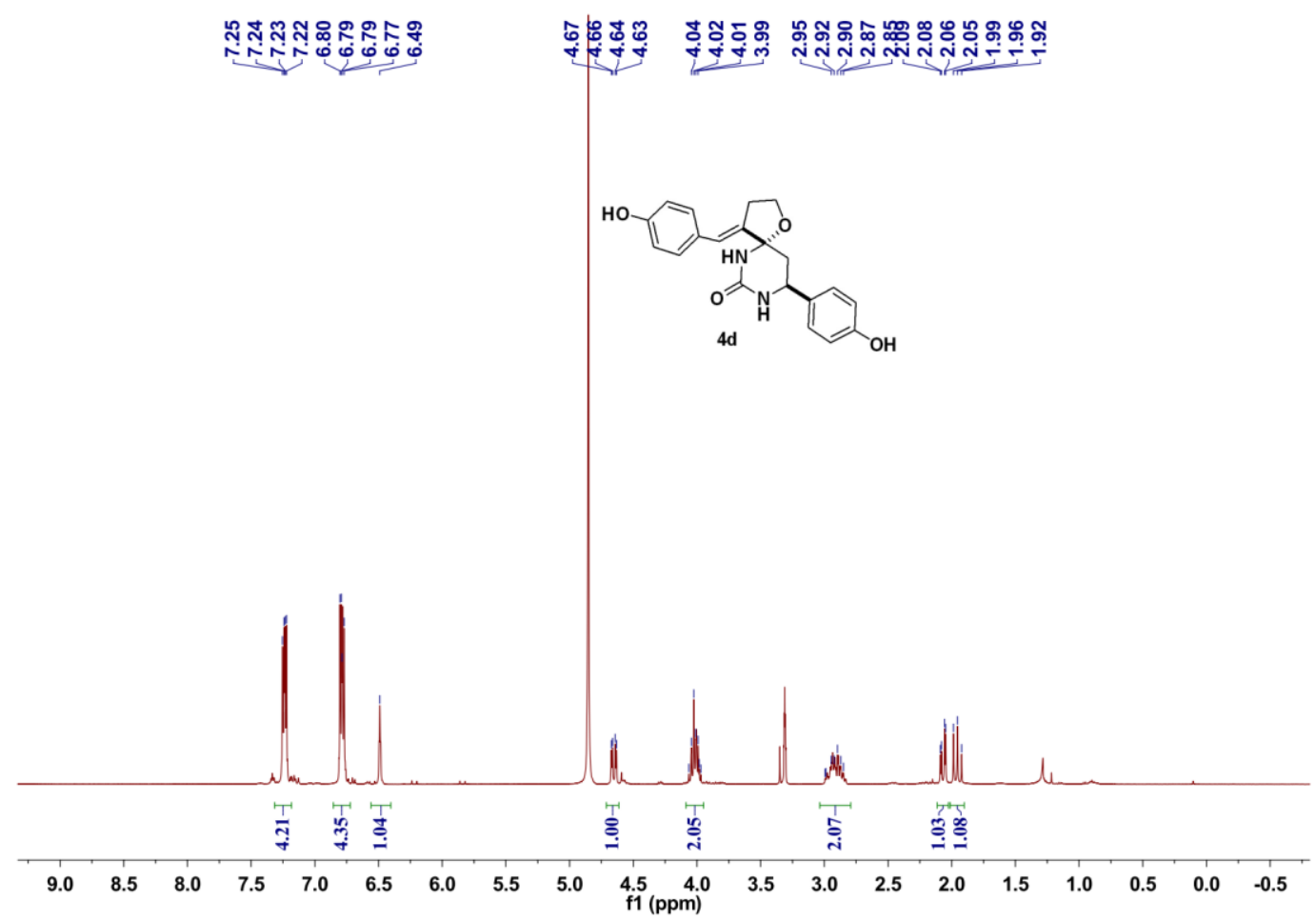

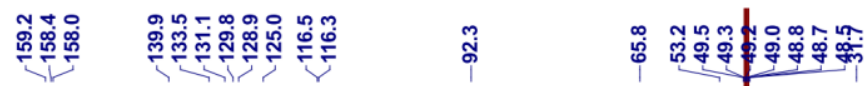
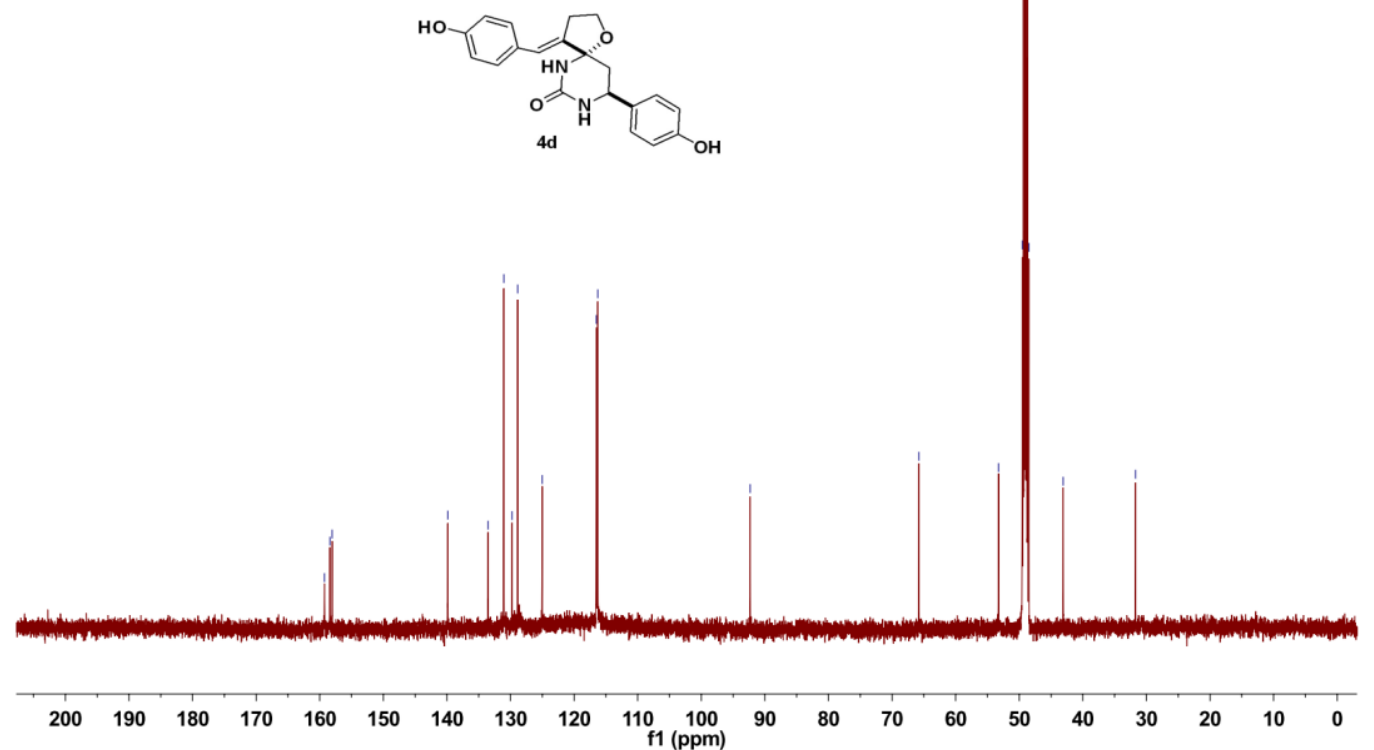


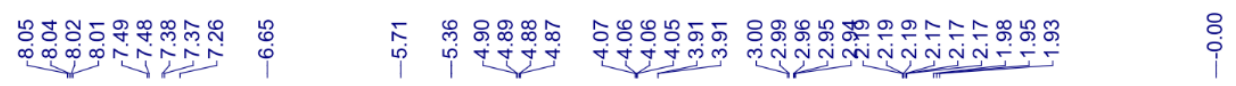

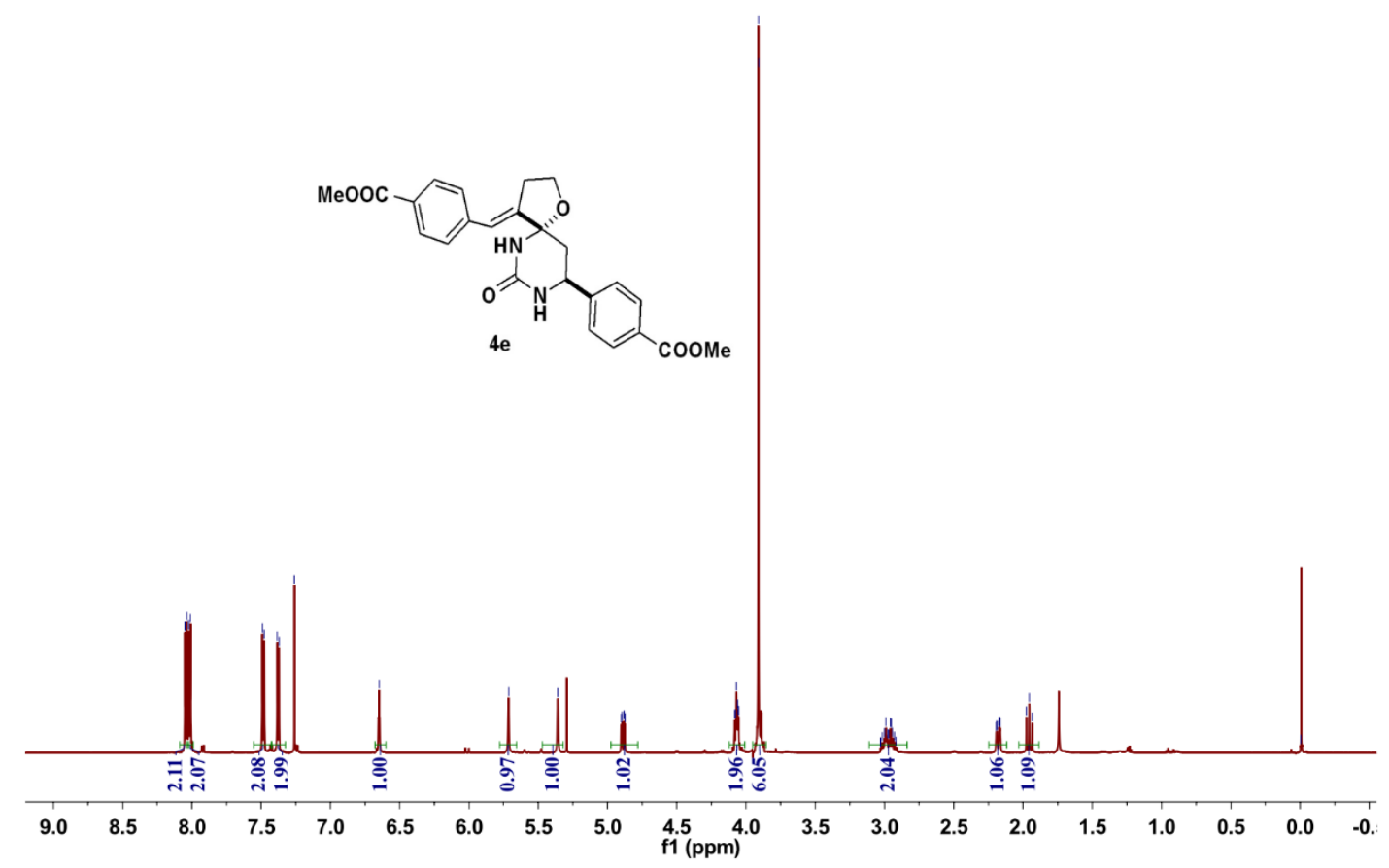

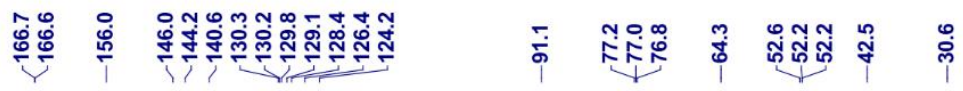
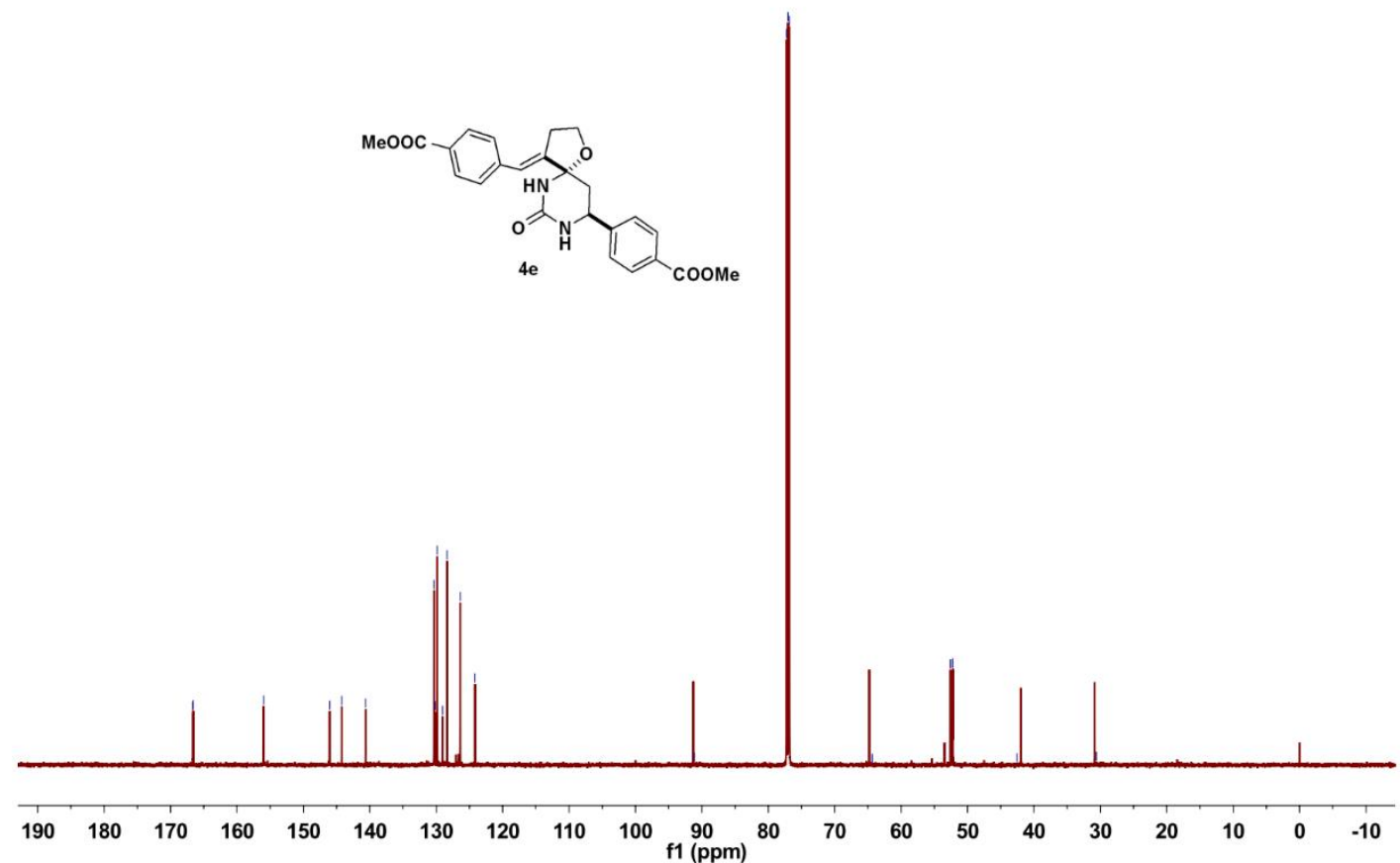


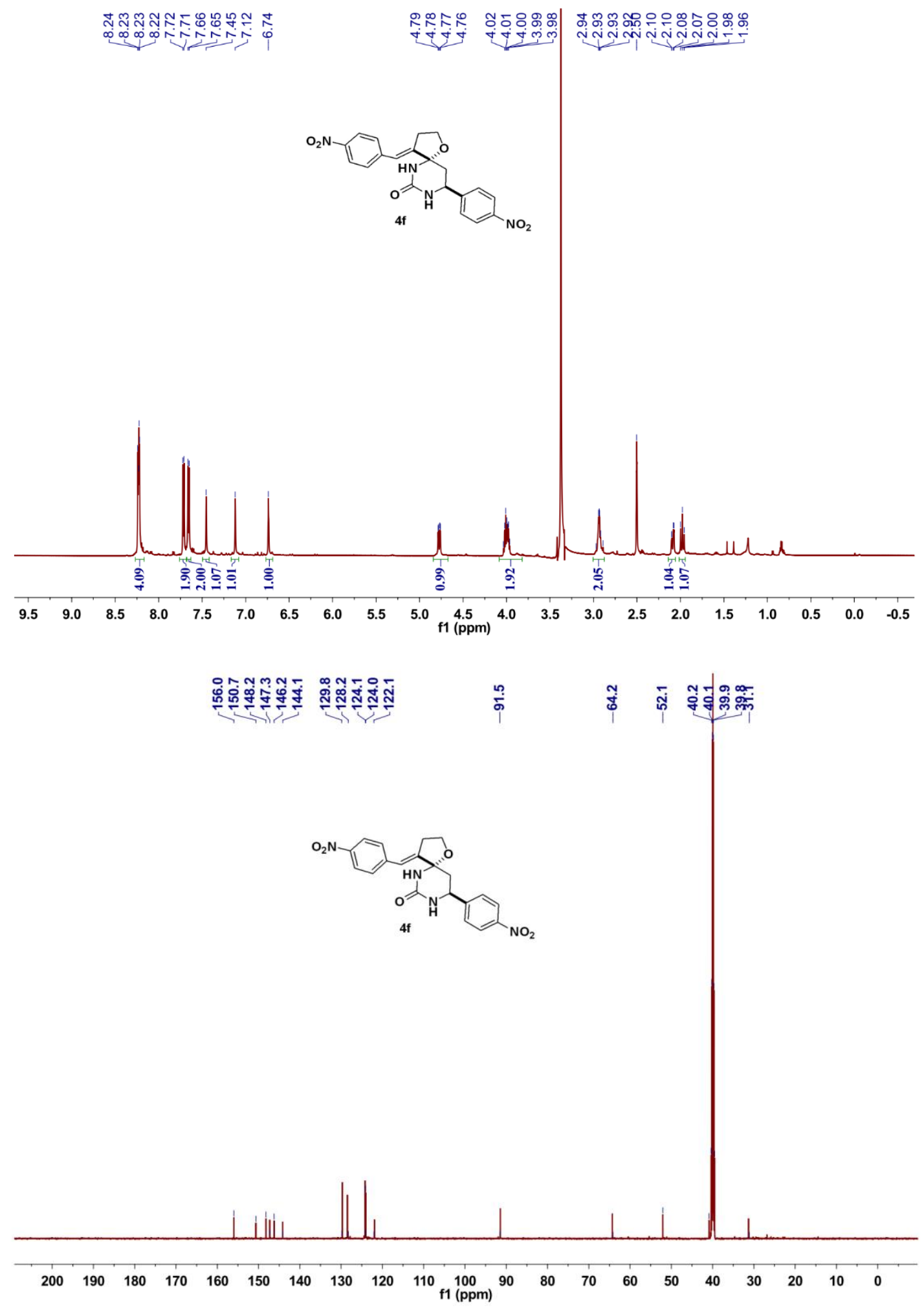



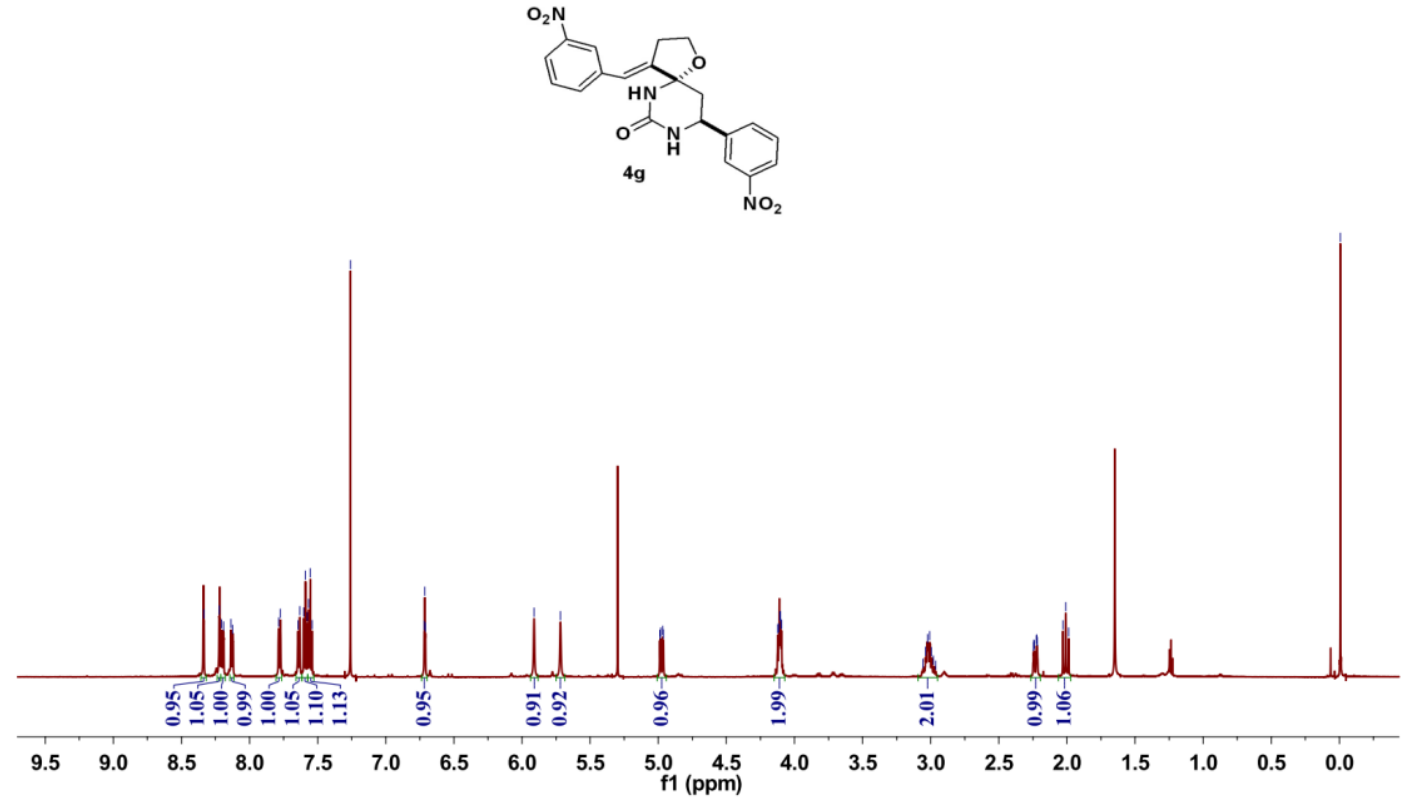

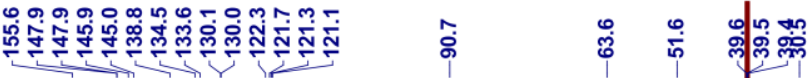
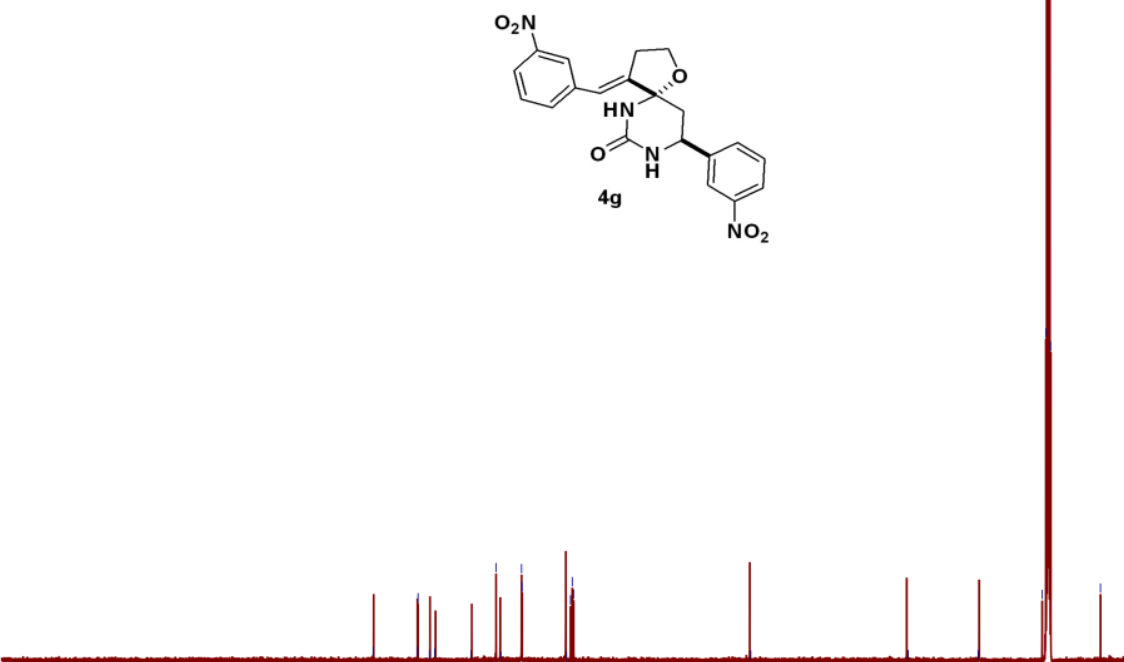

$\begin{array}{llllllllllllllllllllllll}210 & 200 & 190 & 180 & 170 & 160 & 150 & 140 & 130 & 120 & 110 & 100 & 90 & 80 & 70 & 60 & 50 & 40 & 30 & 20 & 10 & 0 & -10 & -2\end{array}$ 


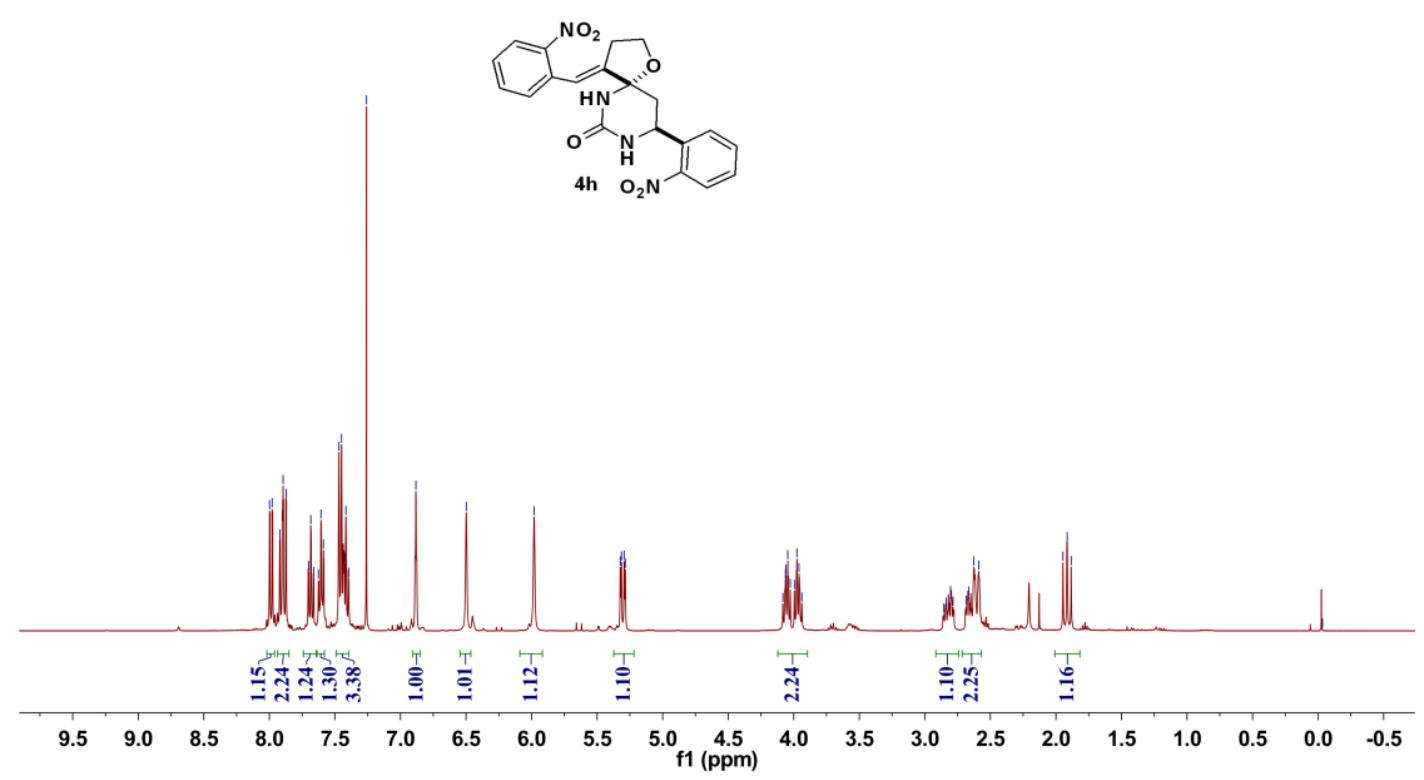

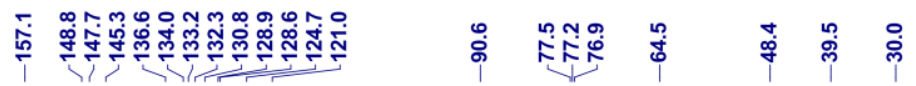
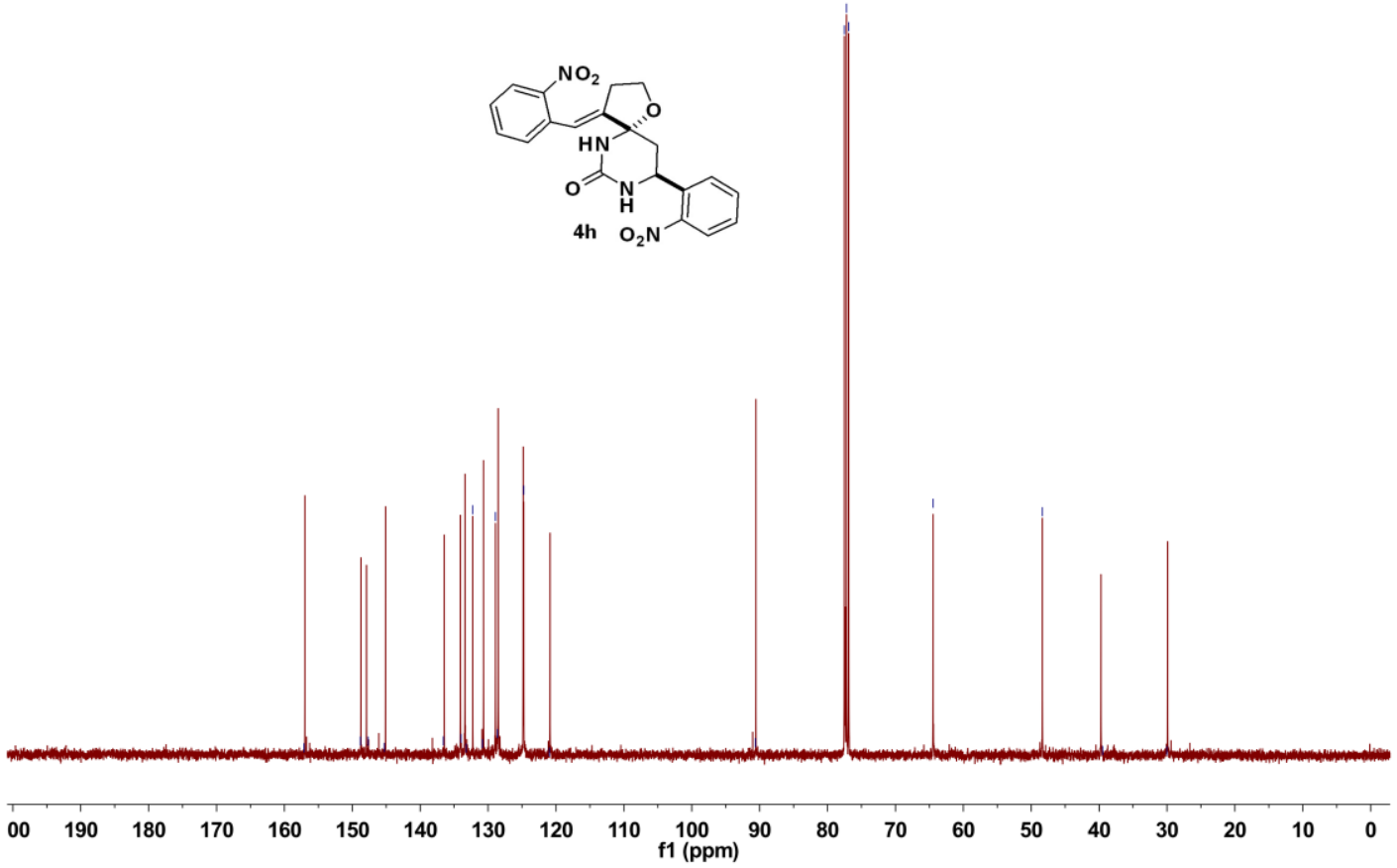


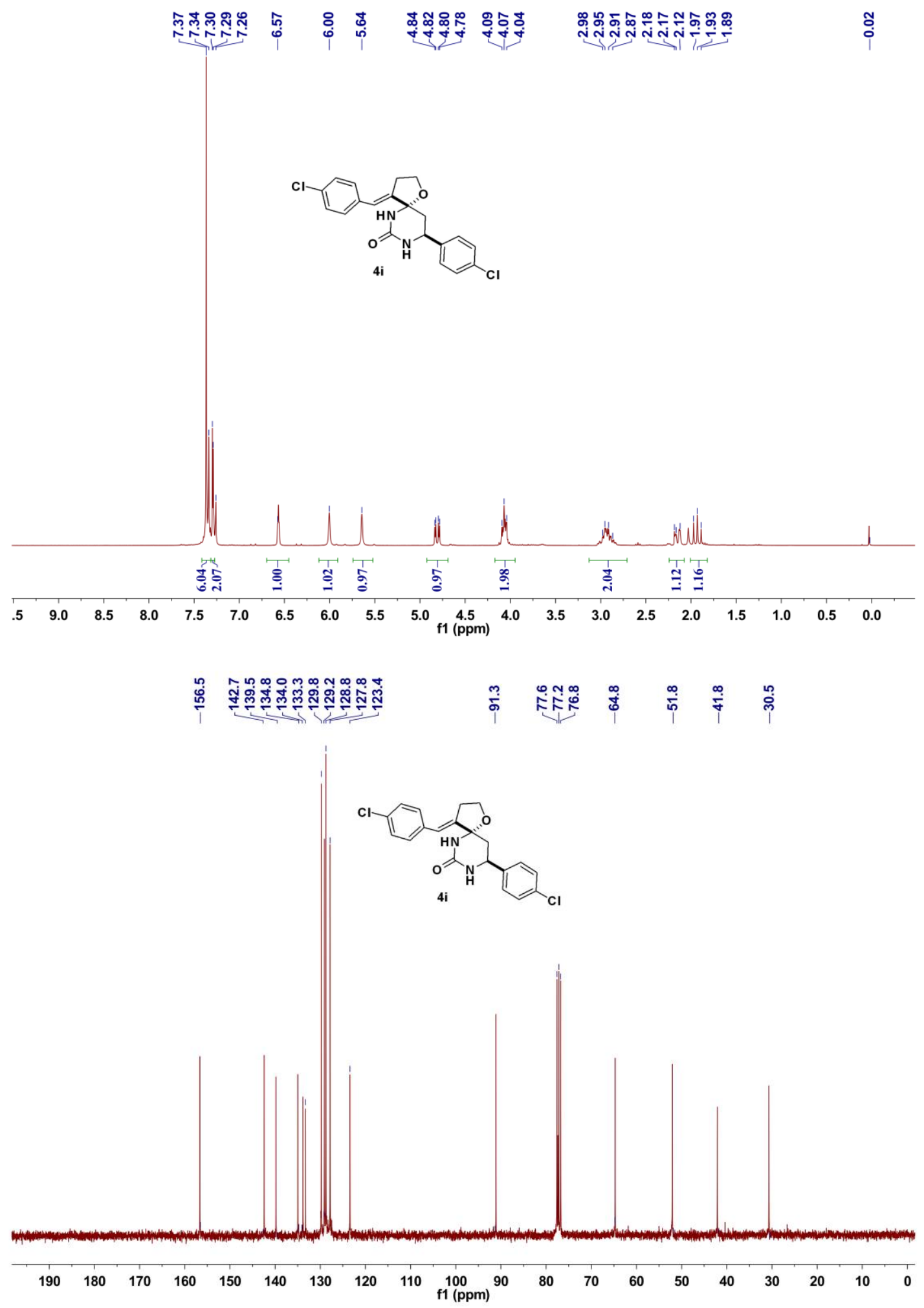




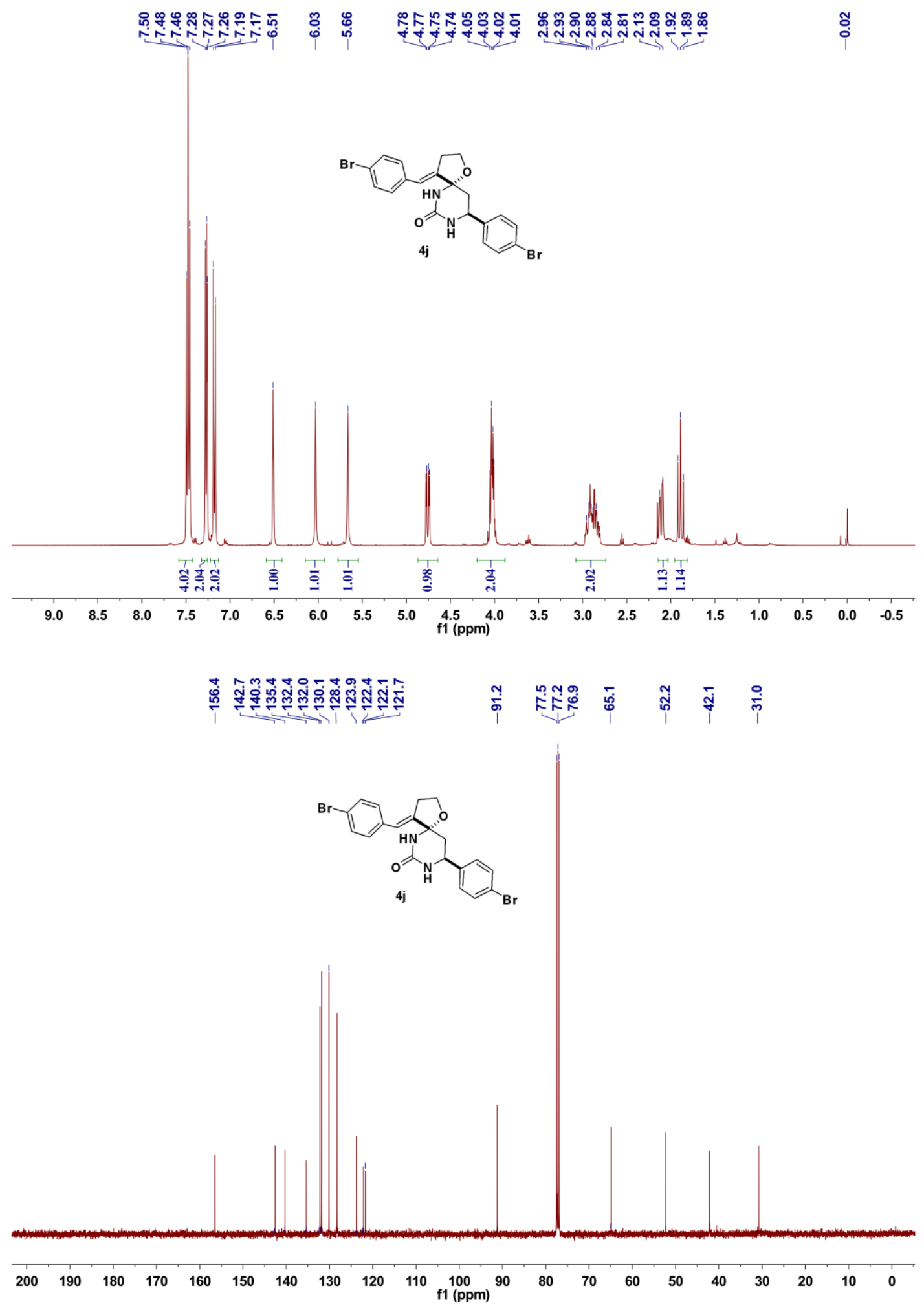



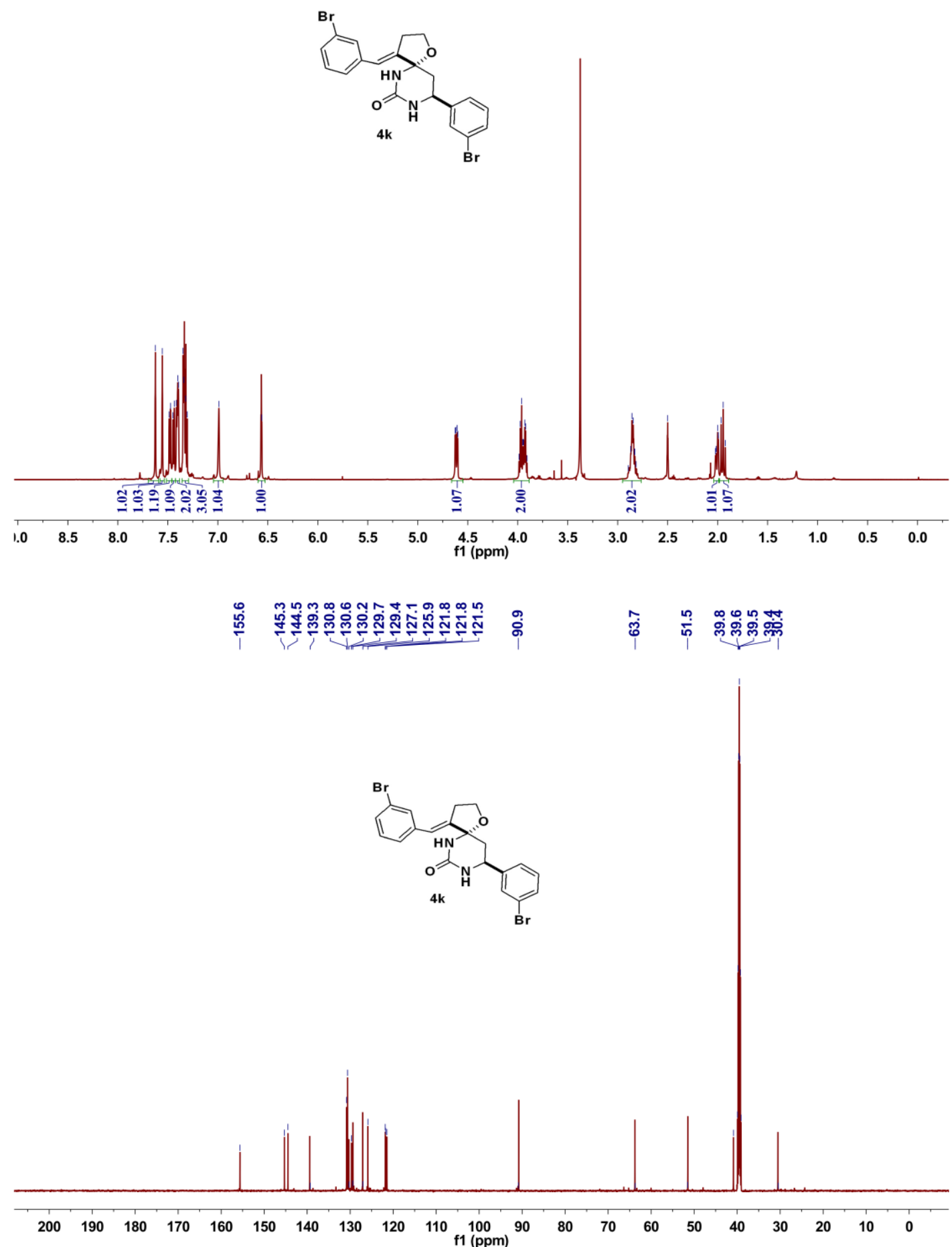


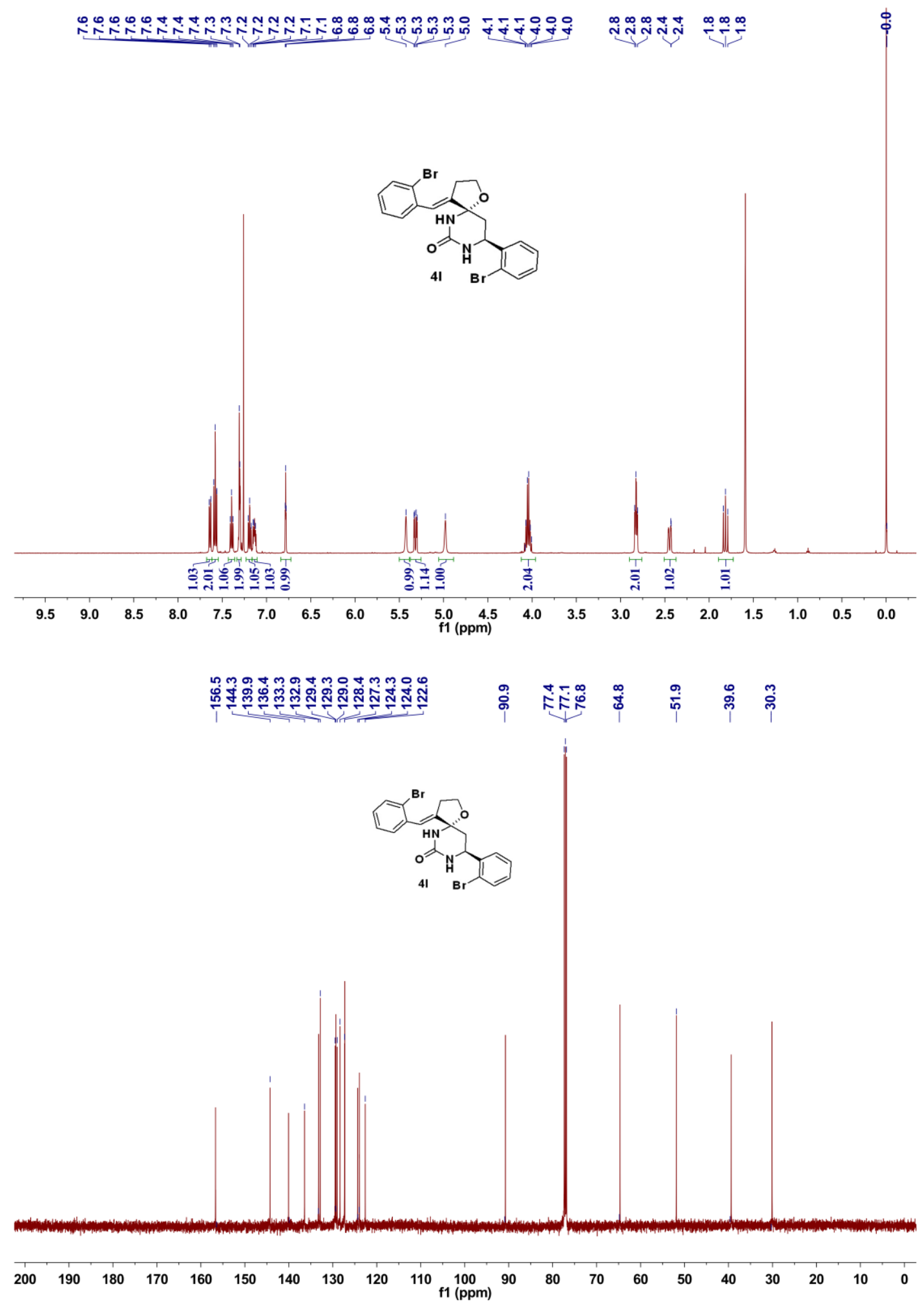




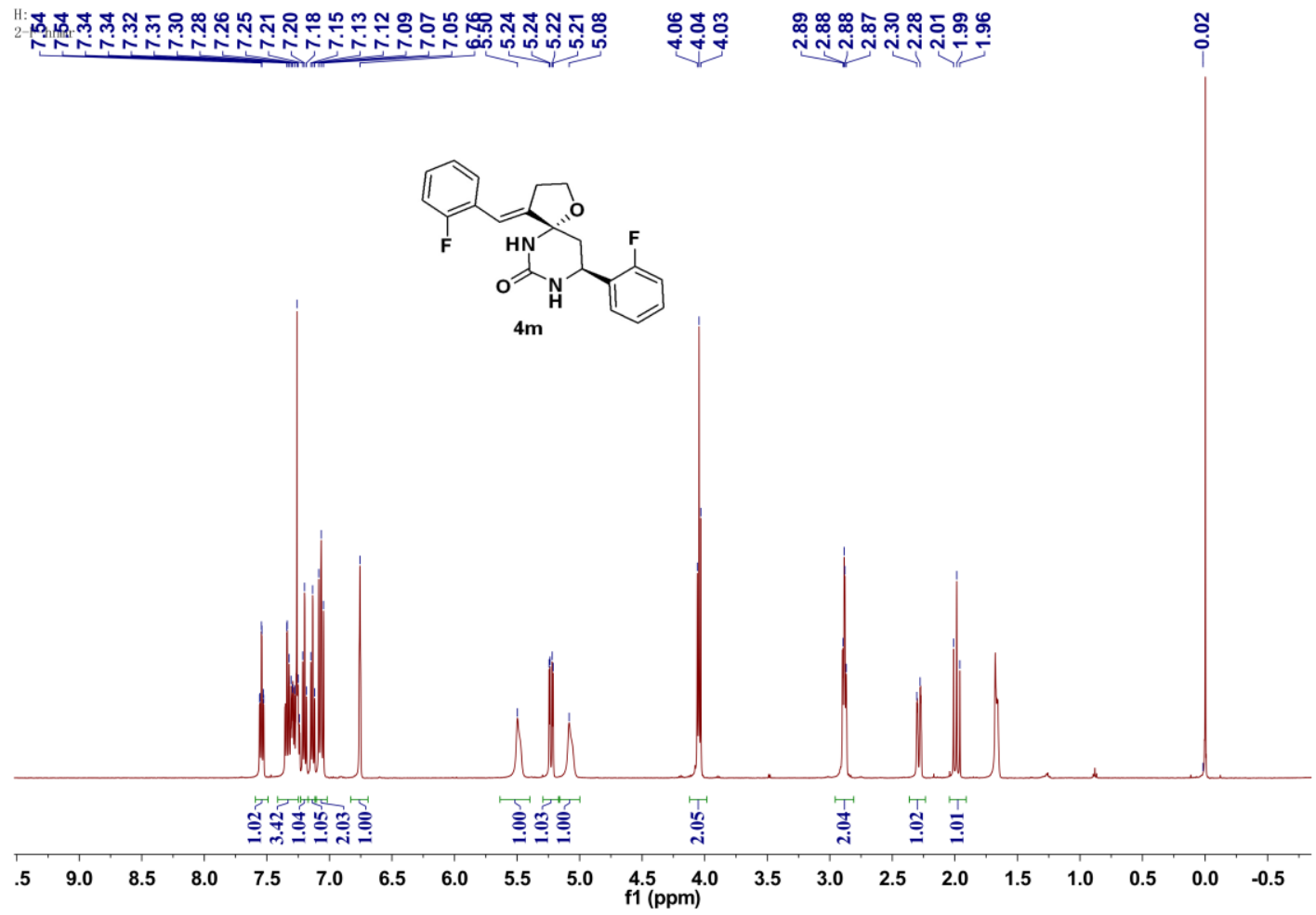

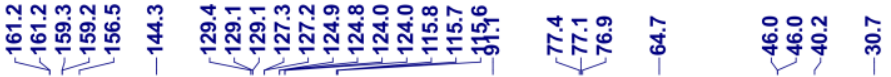

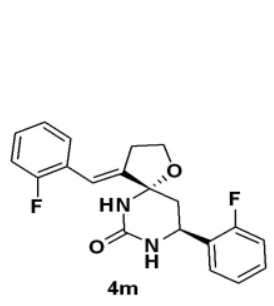




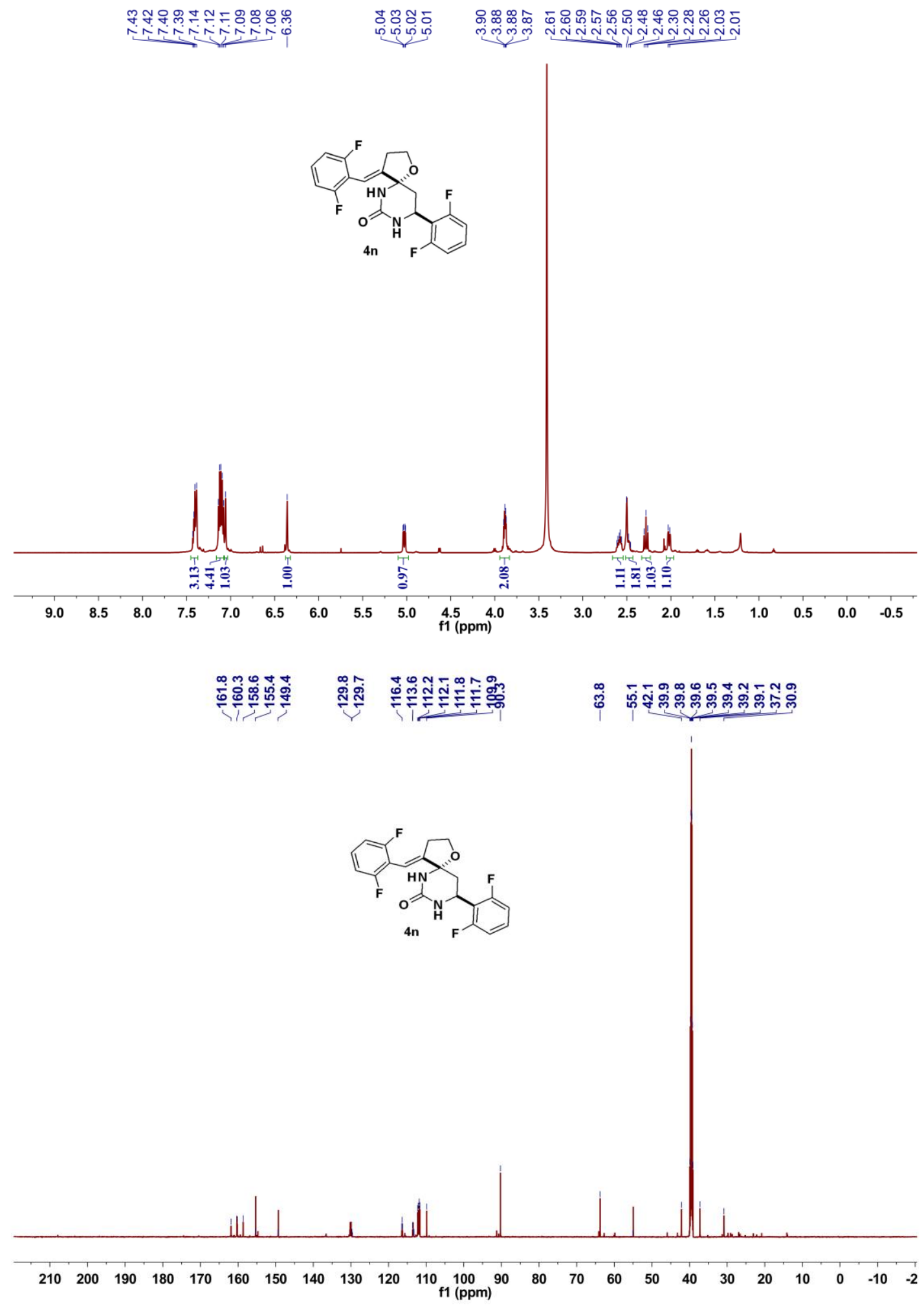



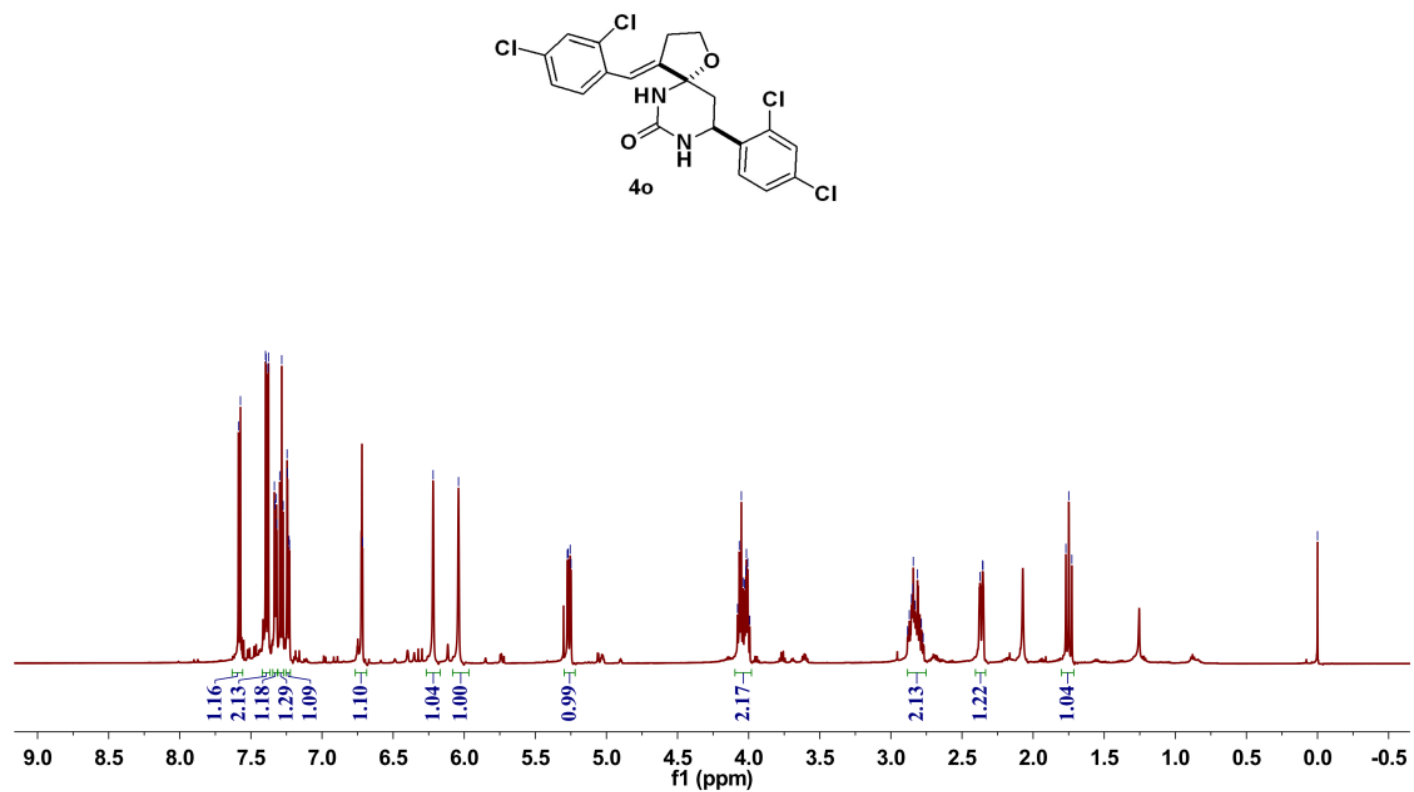

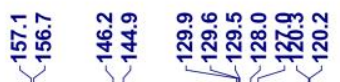

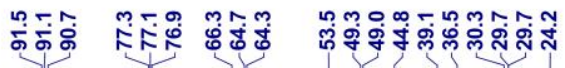
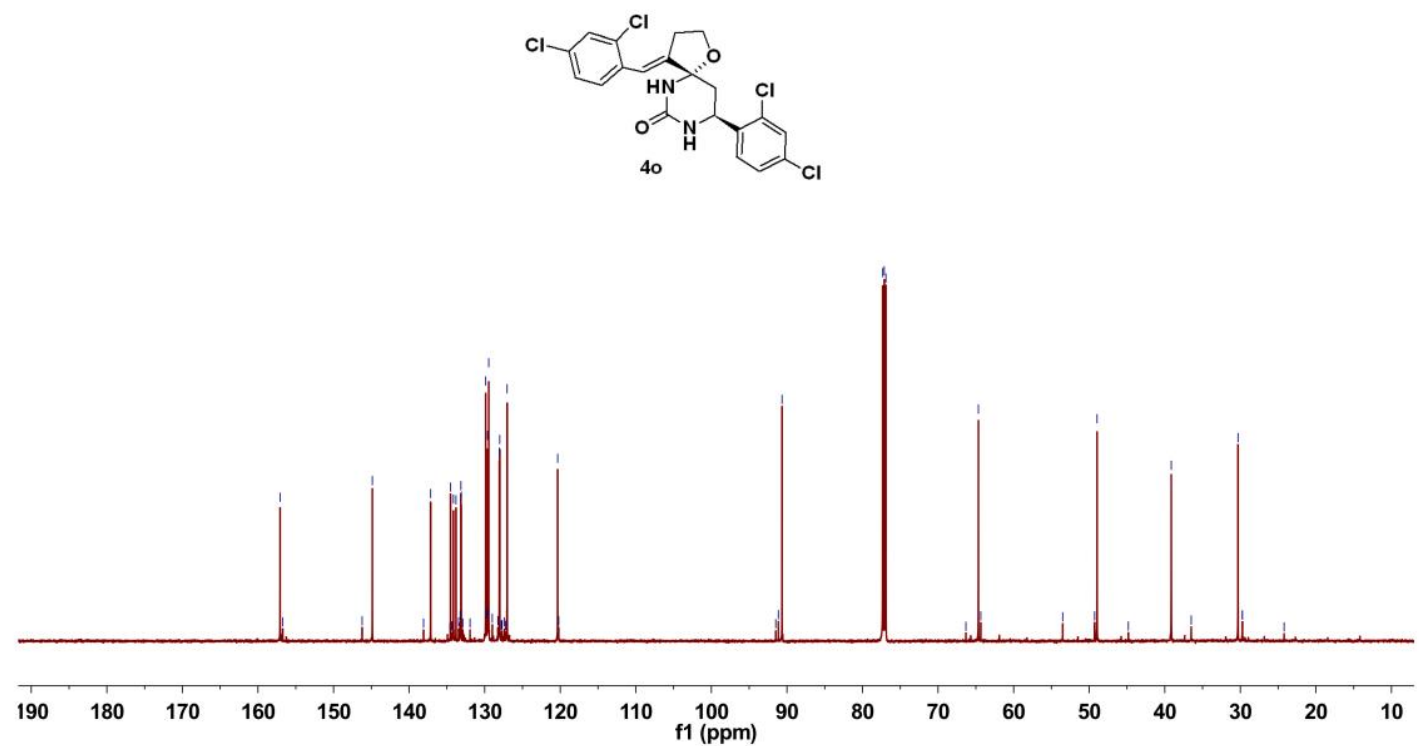


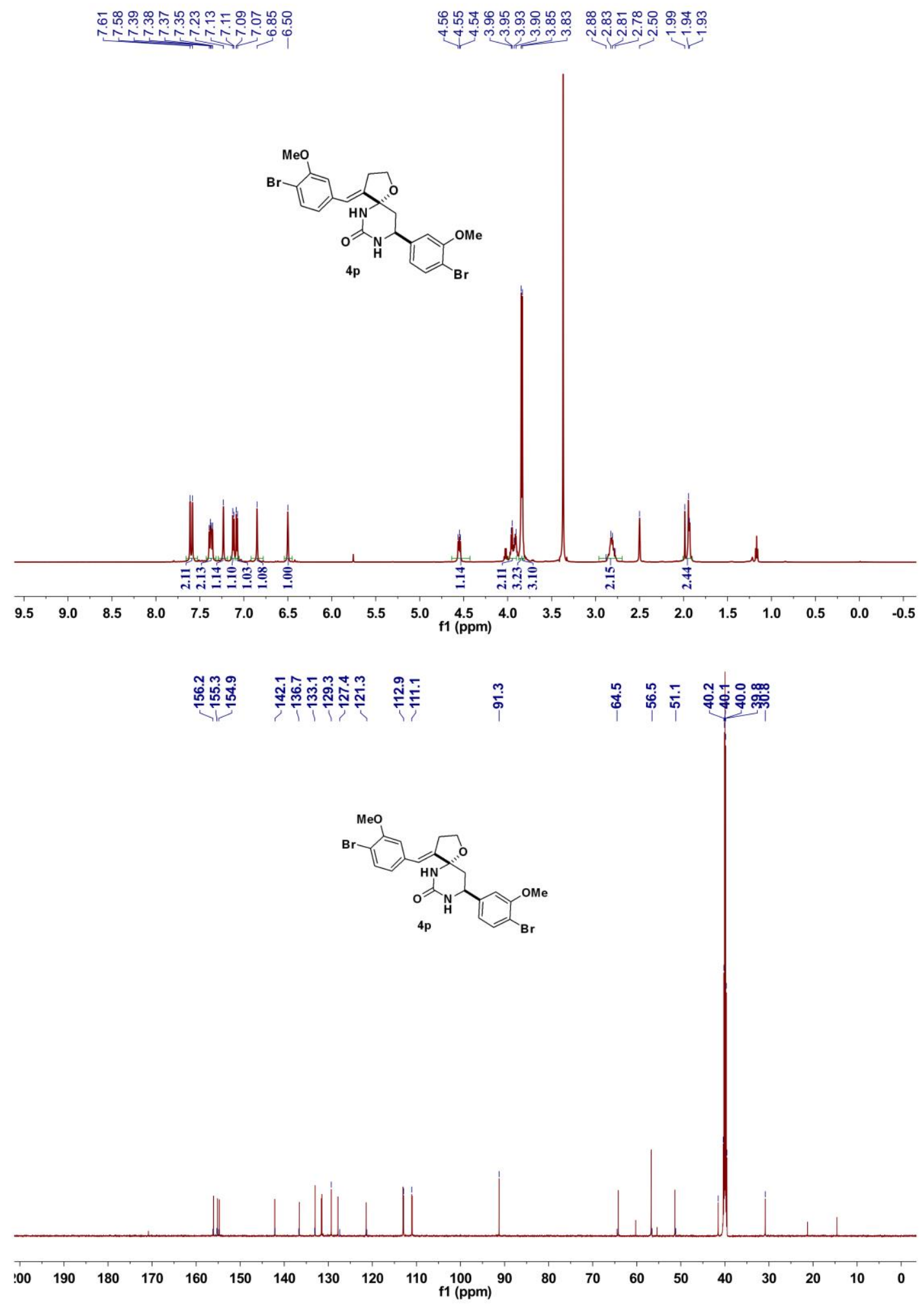




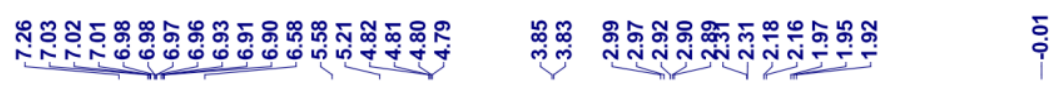

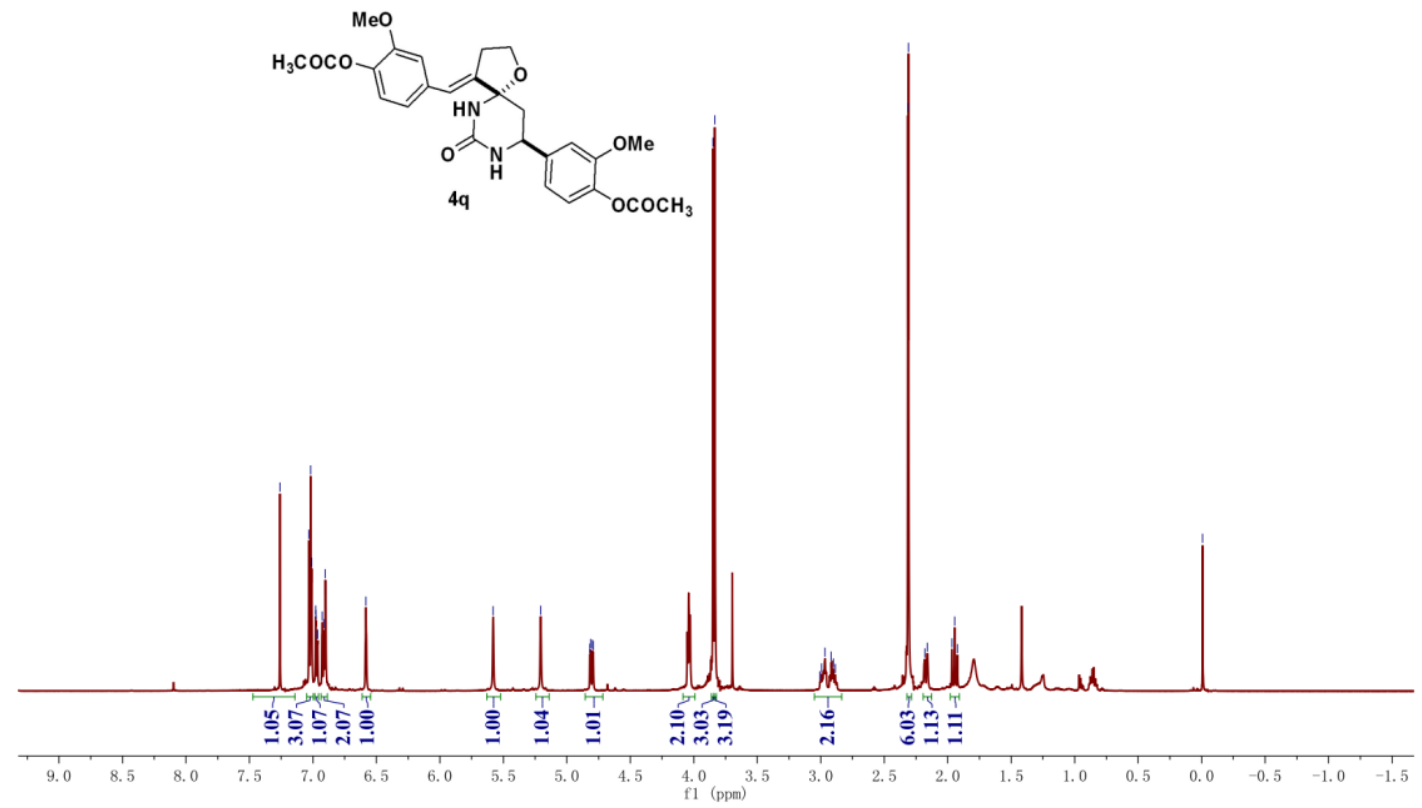

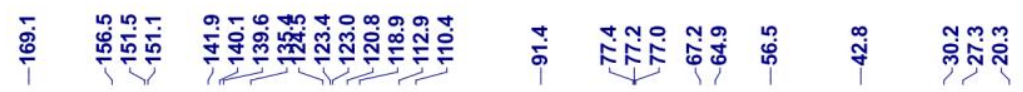
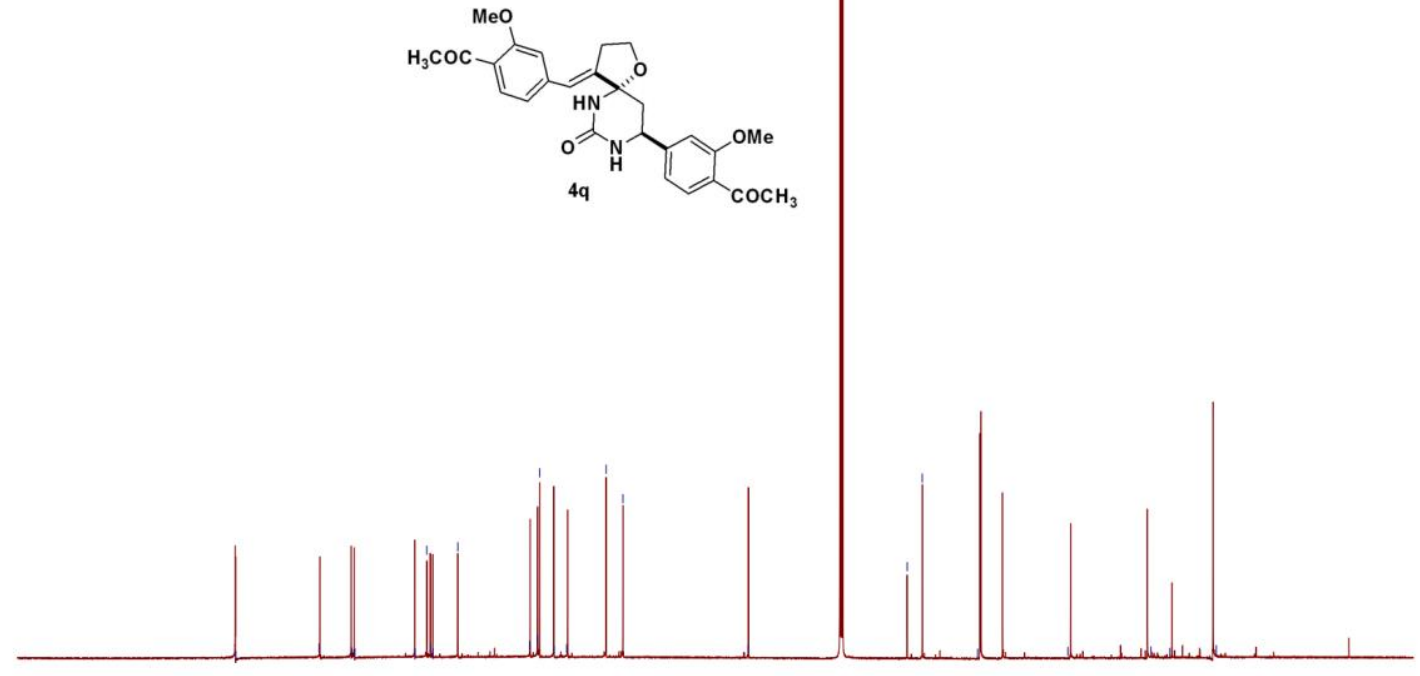

$\begin{array}{llllllllllllllllllll}200 & 190 & 180 & 170 & 160 & 150 & 140 & 130 & 120 & 110 & \begin{array}{l}100 \\ \mathrm{f} 1(\mathrm{ppm})\end{array} & 80 & 70 & 60 & 50 & 40 & 30 & 20 & 10 & 0\end{array}$ 


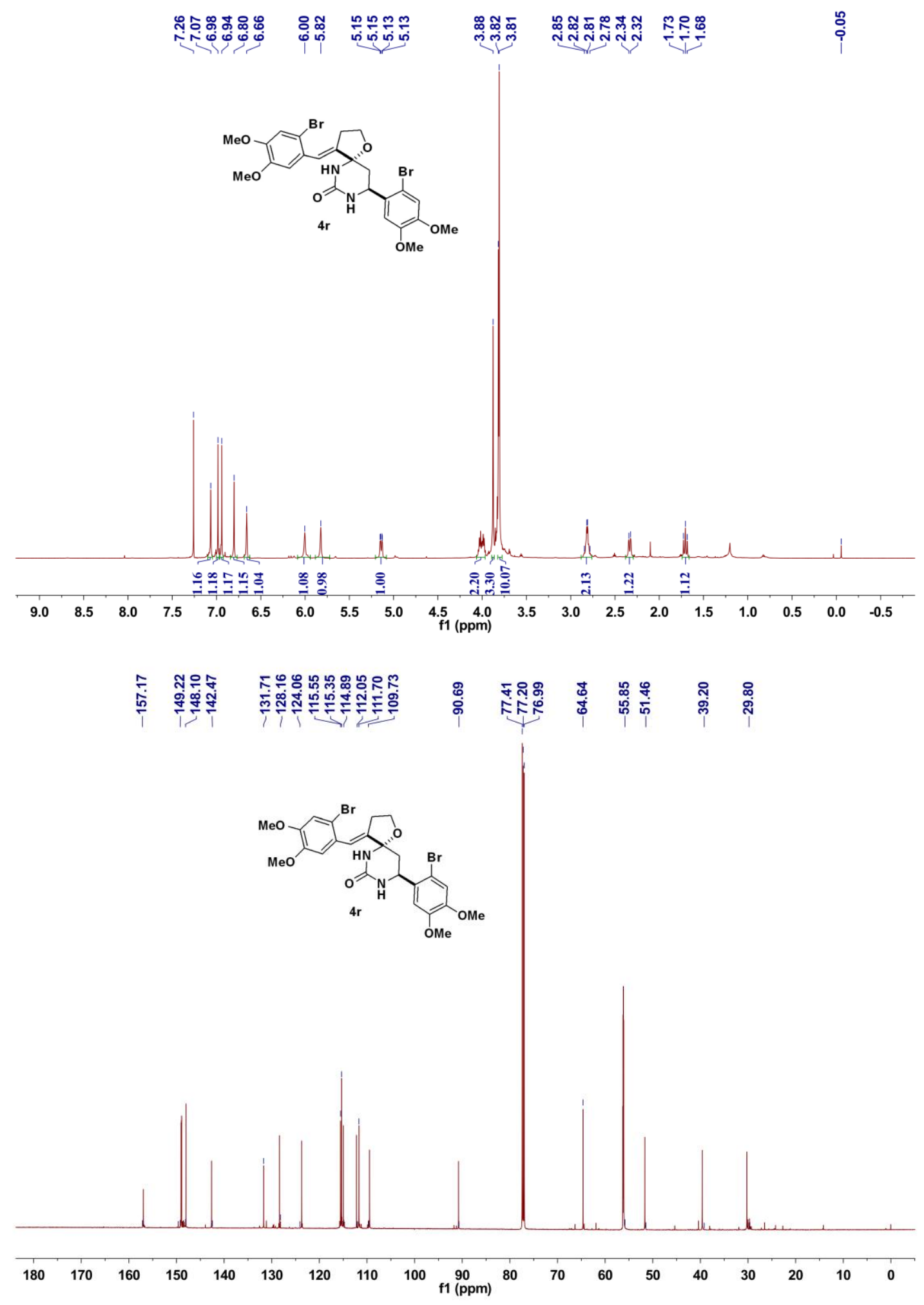




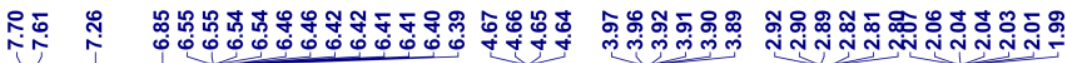

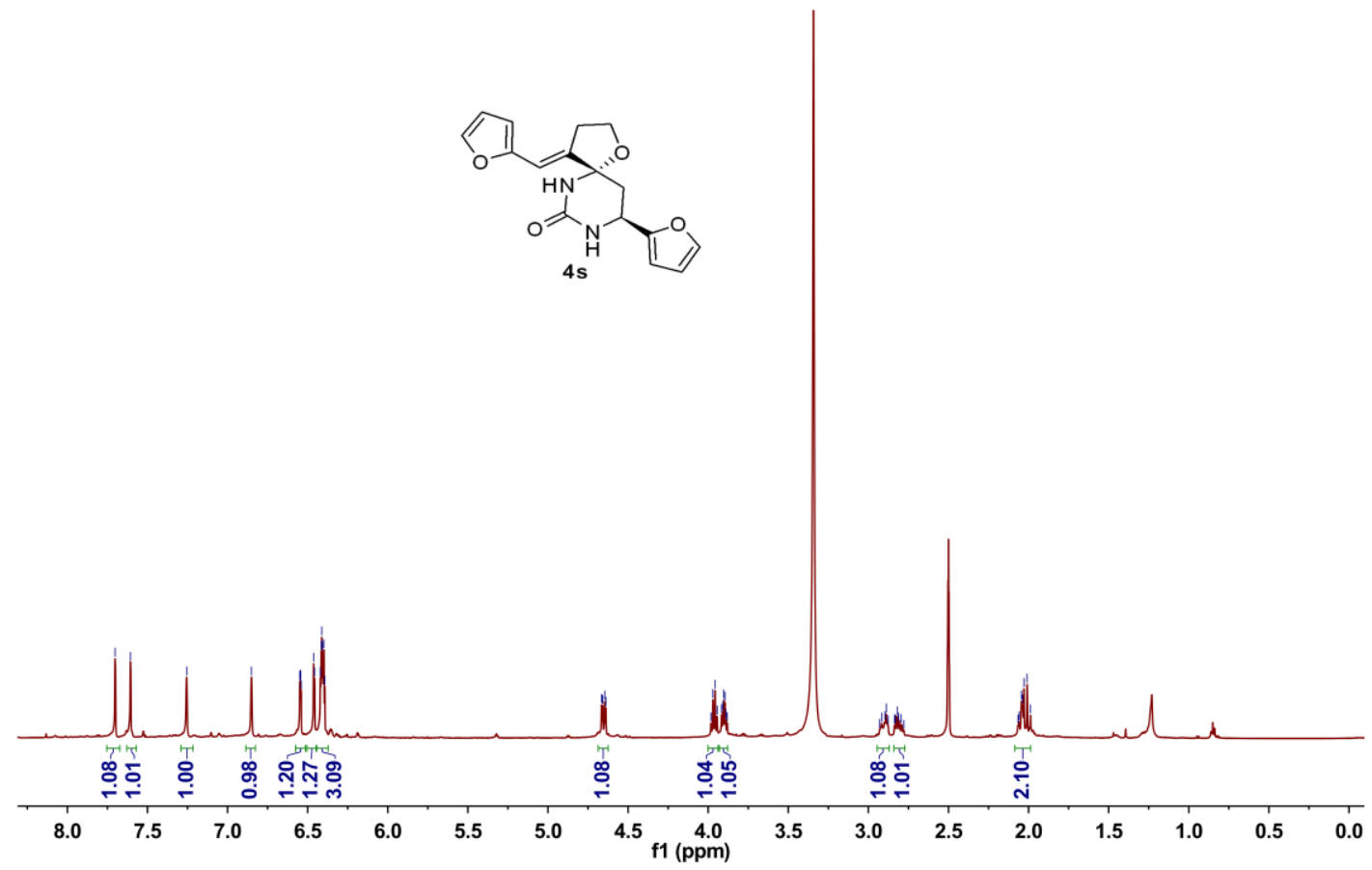

\begin{tabular}{|c|c|c|c|c|c|}
\hline 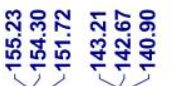 & 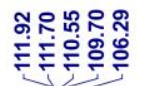 & సั. & $\frac{m}{i}$ & চே. & 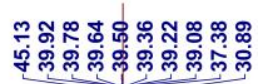 \\
\hline
\end{tabular}
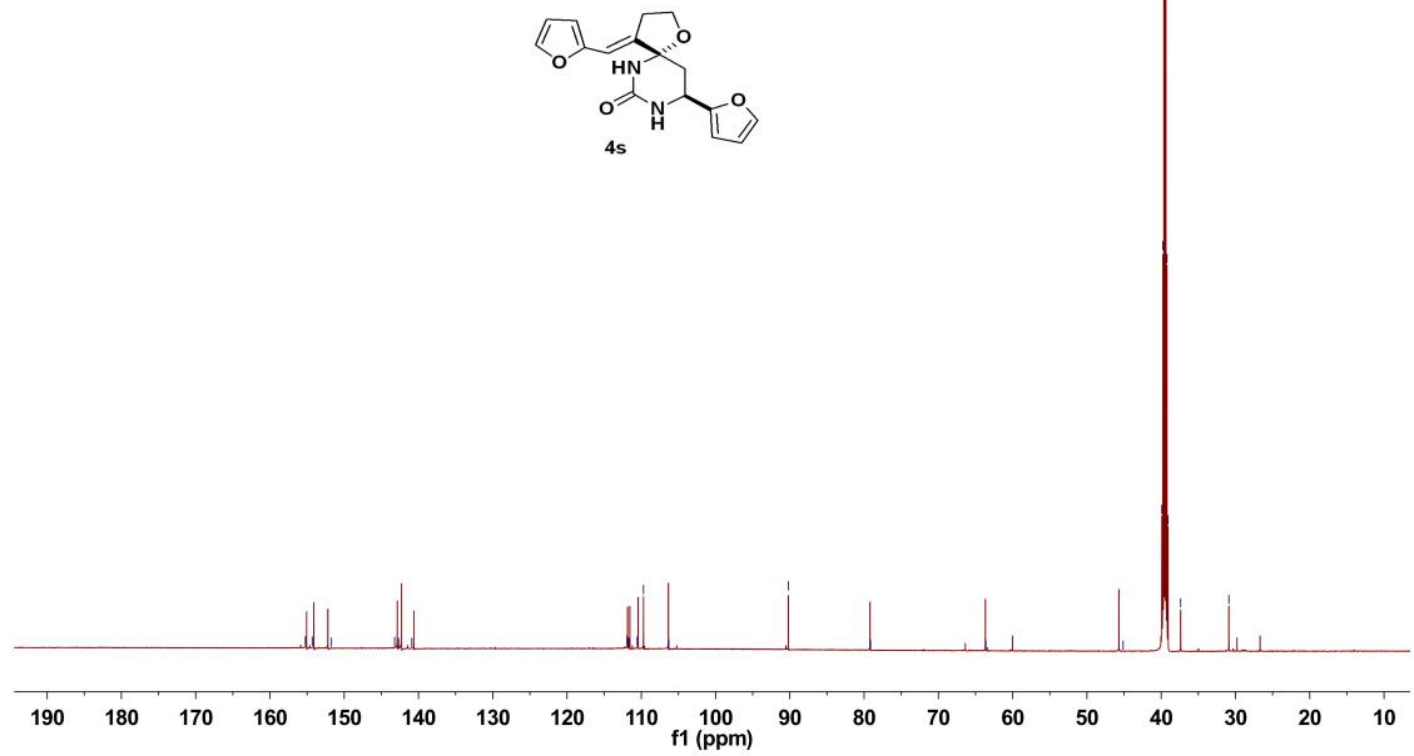


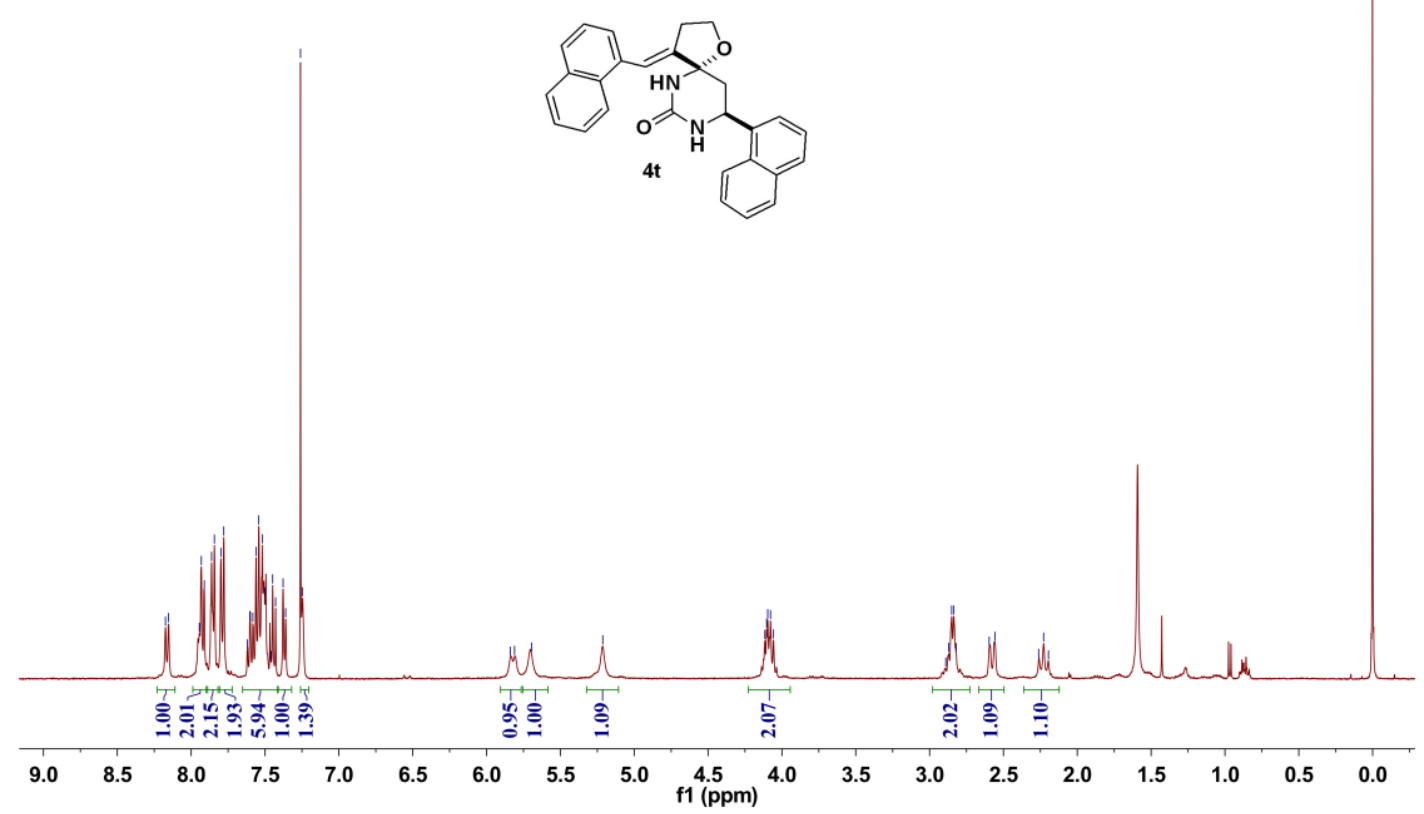

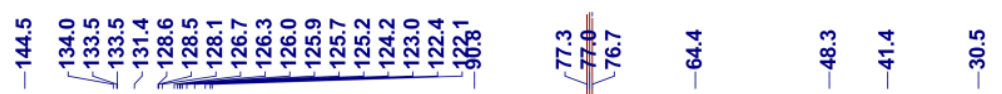
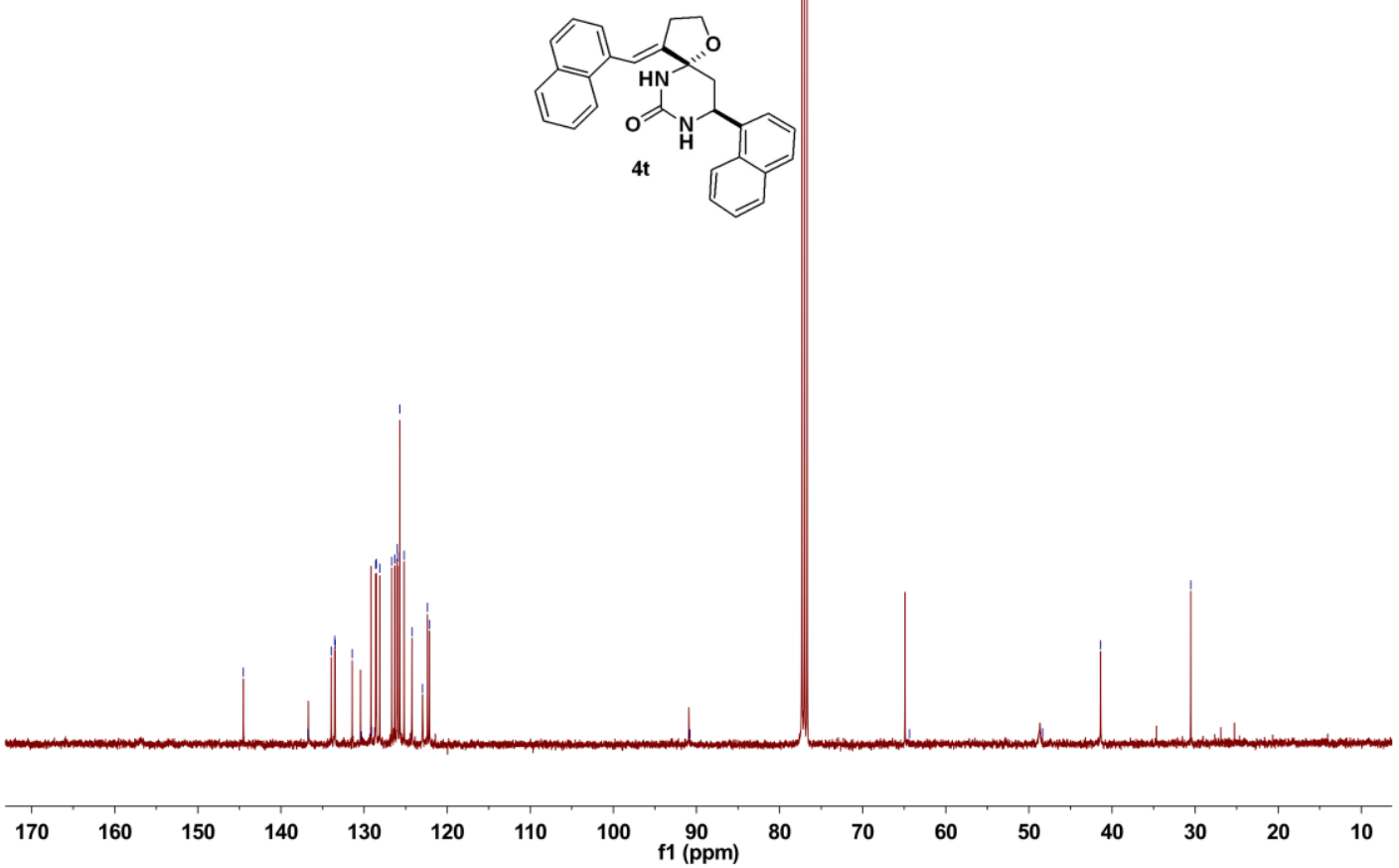


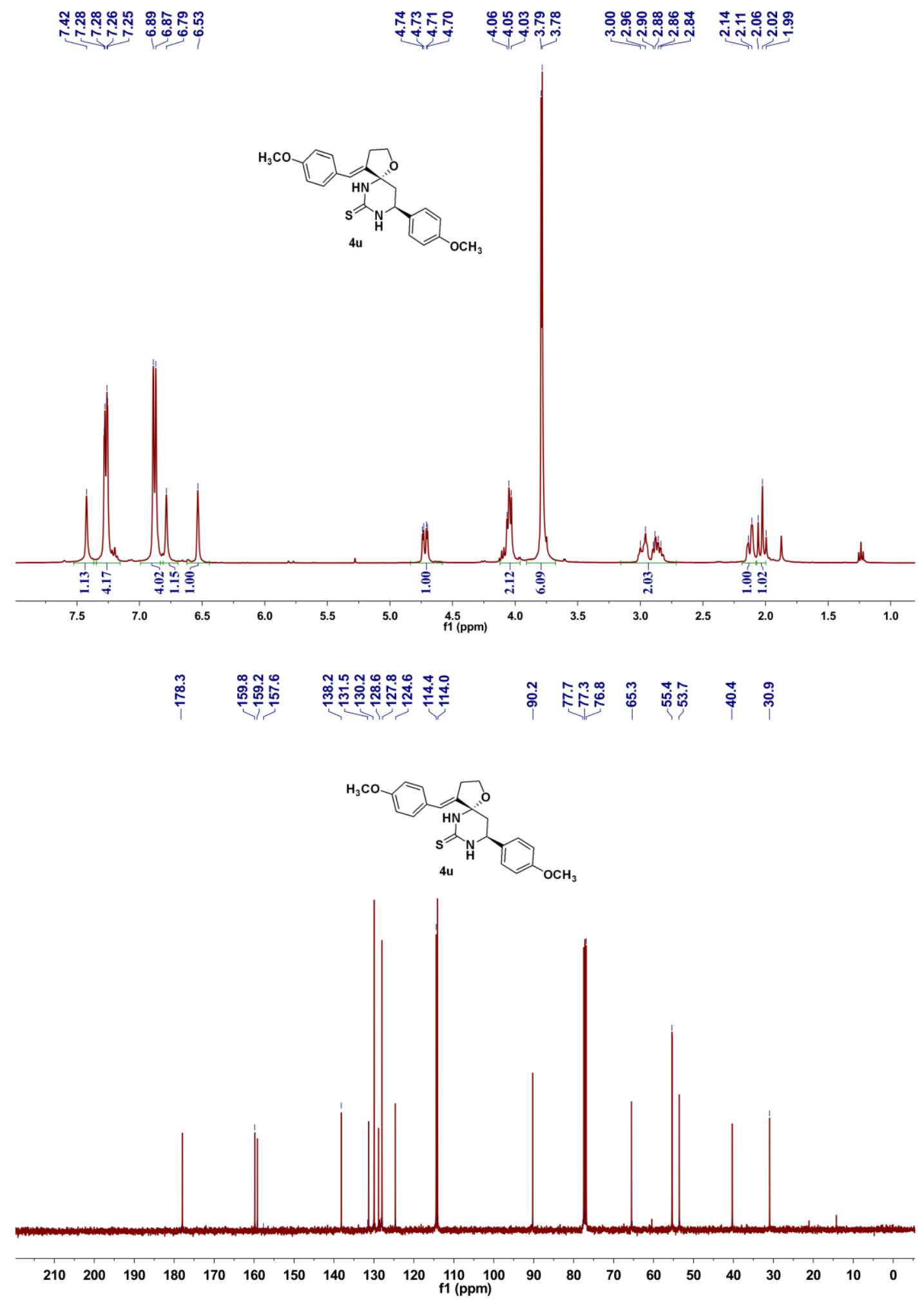



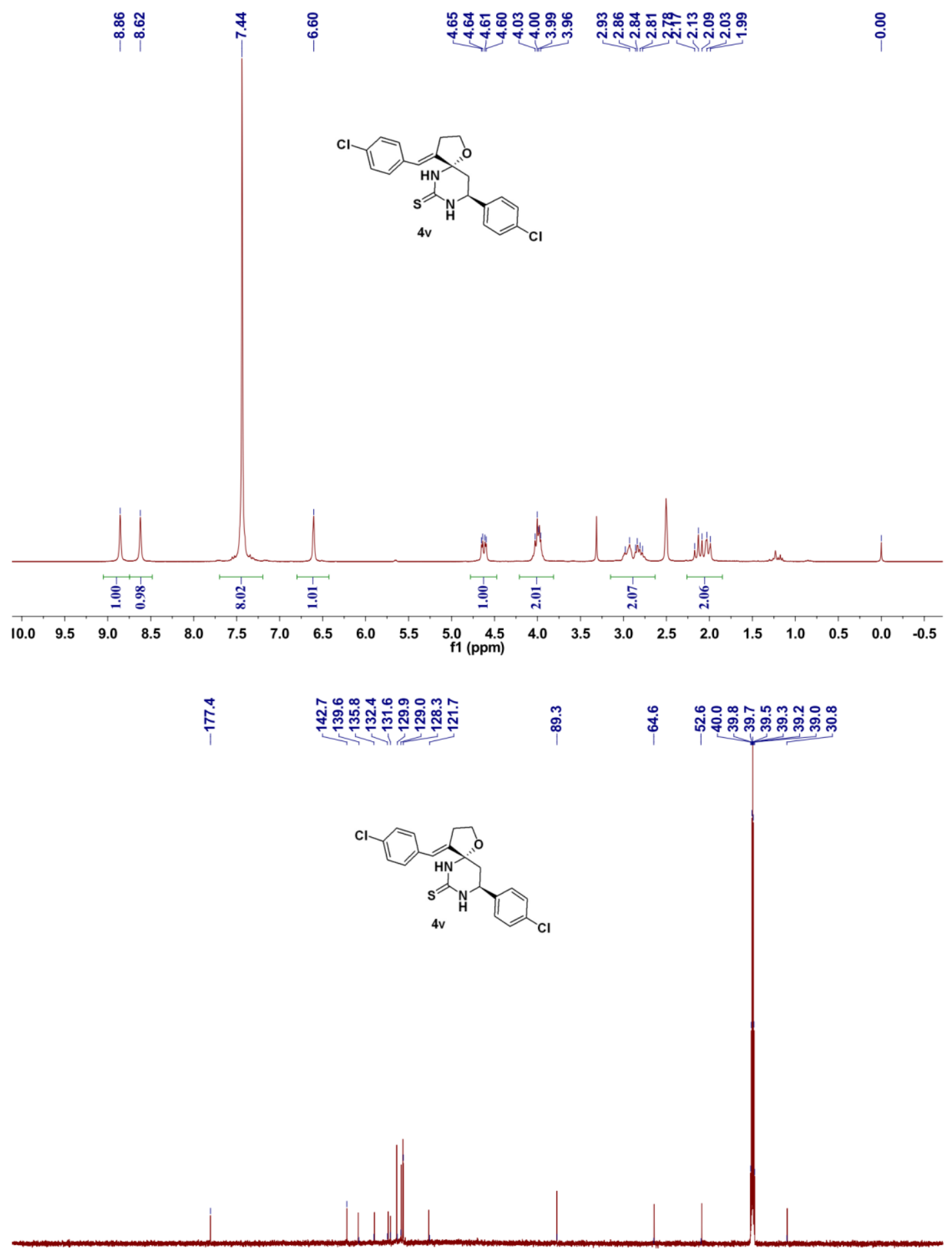

$\begin{array}{lllllllllllllllllllllll}220 & 210 & 200 & 190 & 180 & 170 & 160 & 150 & 140 & 130 & 120 & \begin{array}{c}110 \\ 110\end{array} & 100 & 90 & 80 & 70 & 60 & 50 & 40 & 30 & 20 & 10 & 0\end{array}$ 


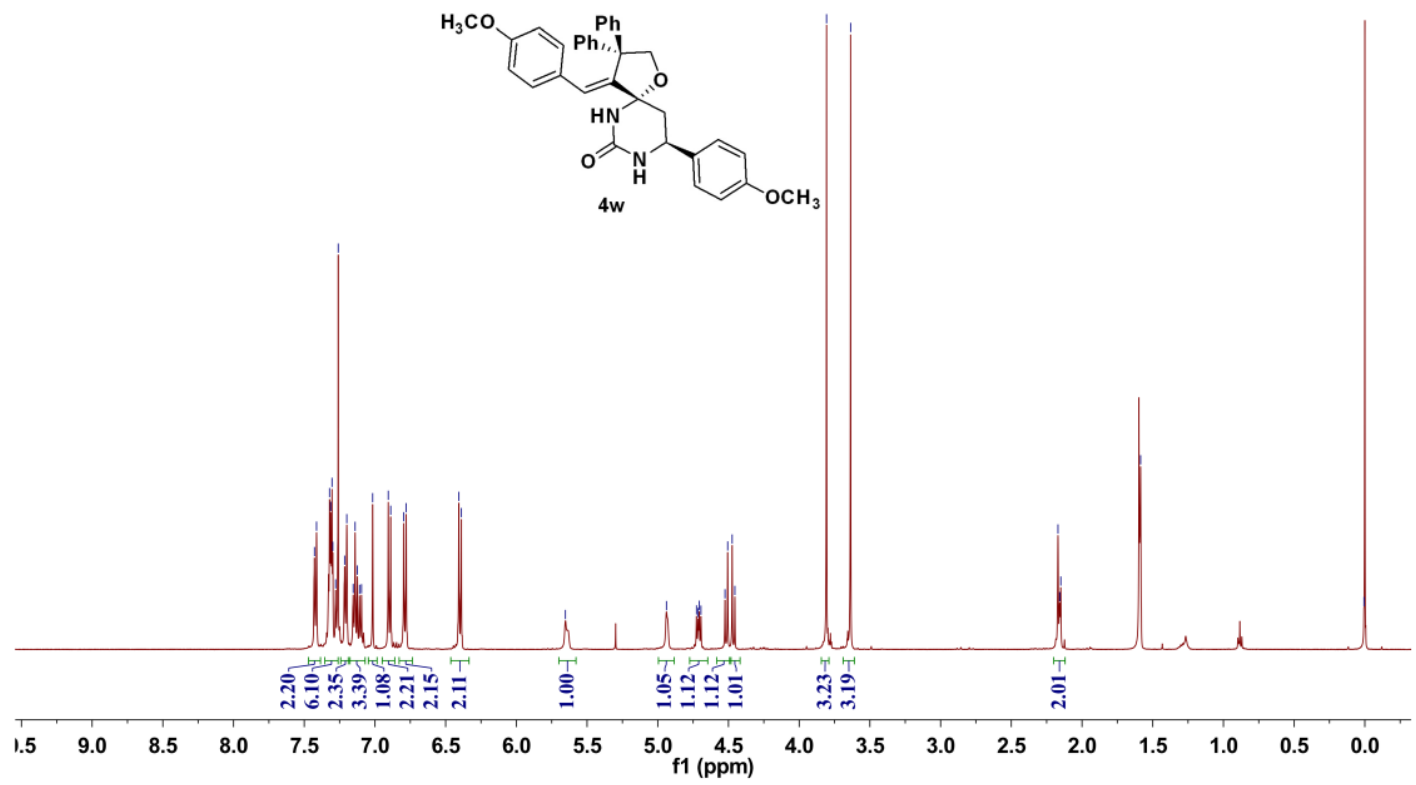

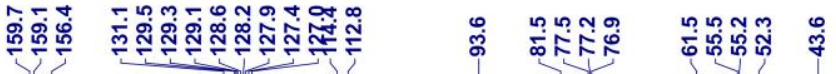
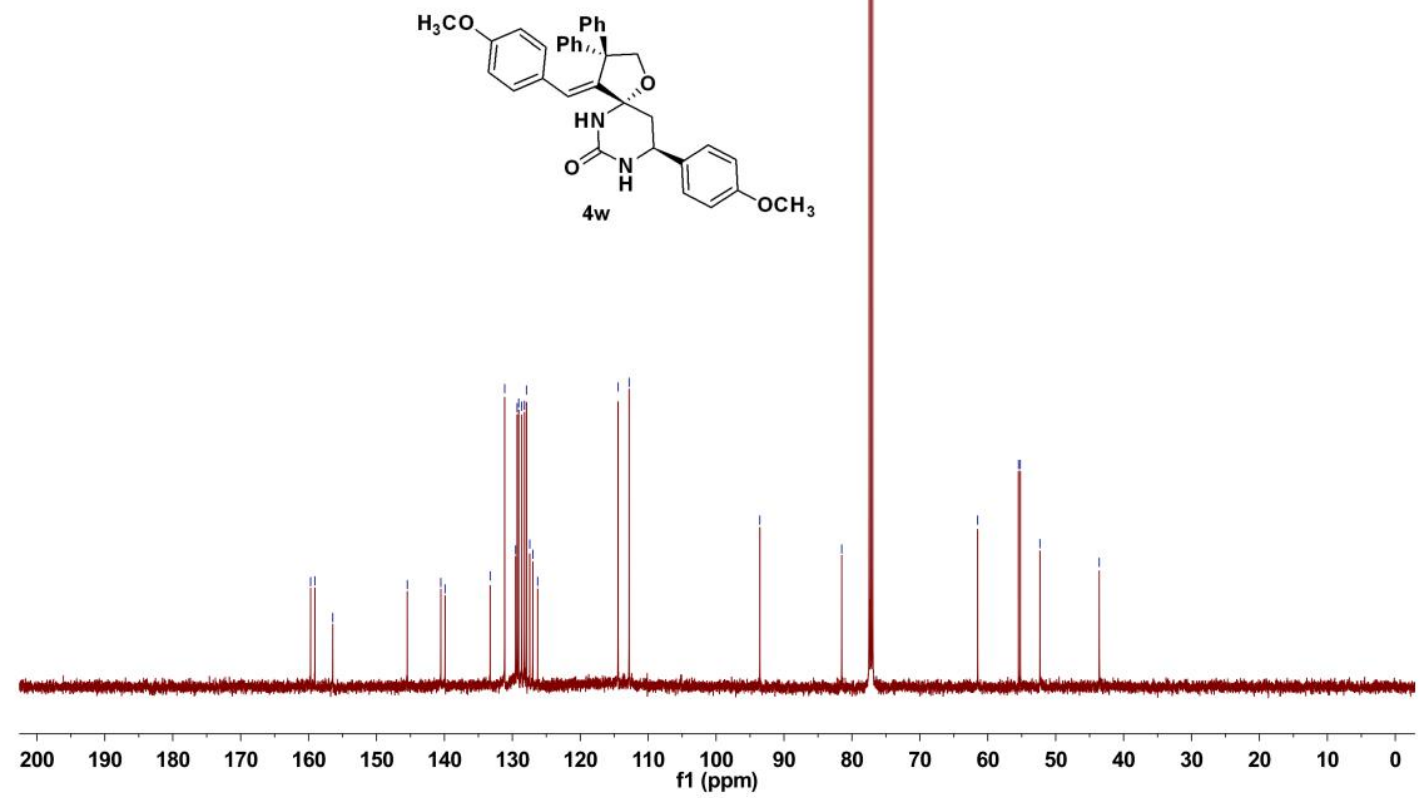


\section{Enantioselective exploration and HPLC spectra}

The enanselective version of this transformation was explored by means of chiral phosphoric acids (CPA) and chiral metal ligand. The results were shown as below. Under the co-catalysis of palladium chloride and CPA, no enantioselectivity was observed. Changing to palladium acetate, the best result was obtained with SPINOL-based CPA ( $33 \%$ yield, $11 \%$ ee). Chiral ligand proved to be also valid, although only $5 \%$ ee was obtained by now.
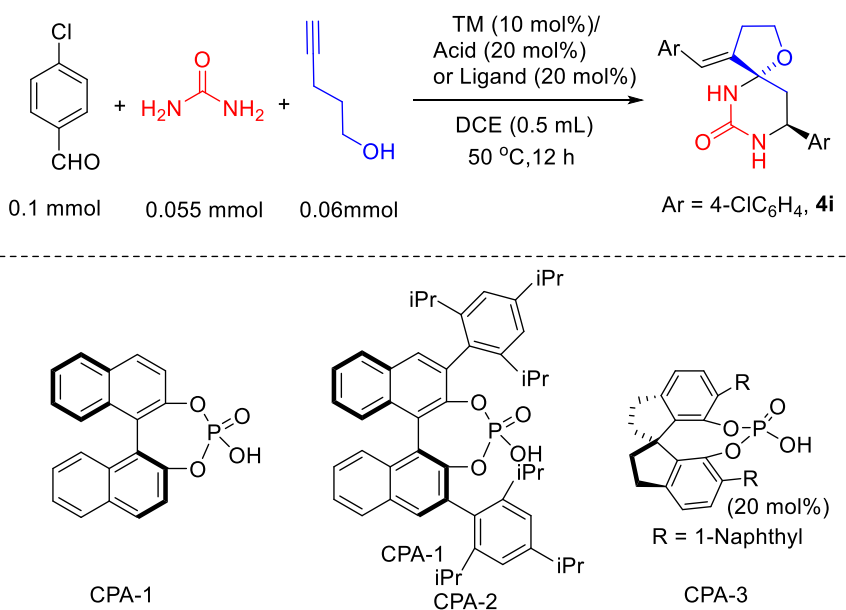

CPA-1 CPA-3

\begin{tabular}{|c|c|c|c|c|c|}
\hline entry & $\mathrm{TM}$ & acid & ligand & yield/\% & ee/\% \\
\hline 1 & $\mathrm{PdCl}_{2}$ & $\mathrm{CPA}-1$ & -- & 35 & 0 \\
\hline 2 & $\mathrm{PdCl}_{2}$ & $\mathrm{CPA}-2$ & -- & 32 & 0 \\
\hline 3 & $\mathrm{Pd}(\mathrm{OAc})_{2}$ & $\mathrm{CPA}-1$ & -- & 28 & -7 \\
\hline 4 & $\mathrm{Pd}(\mathrm{OAc})_{2}$ & $\mathrm{CPA}-2$ & -- & 24 & 0 \\
\hline 5 & $\mathrm{Pd}(\mathrm{OAc})_{2}$ & $\mathrm{CPA}-3$ & -- & 33 & 11 \\
\hline 6 & $\mathrm{PdCl}_{2}$ & $\mathrm{TFA}$ & $\mathrm{R}-\mathrm{BINAP}$ & 43 & 5 \\
\hline
\end{tabular}


HPLC spectra of racemic reaction with palladium chloride and TFA

(HPLC analysis: IB column, $25^{\circ} \mathrm{C}$, hexans $/ \mathrm{iPrOH}=70 / 30,0.7 \mathrm{~mL} / \mathrm{min}, \lambda=254 \mathrm{~nm}$ )

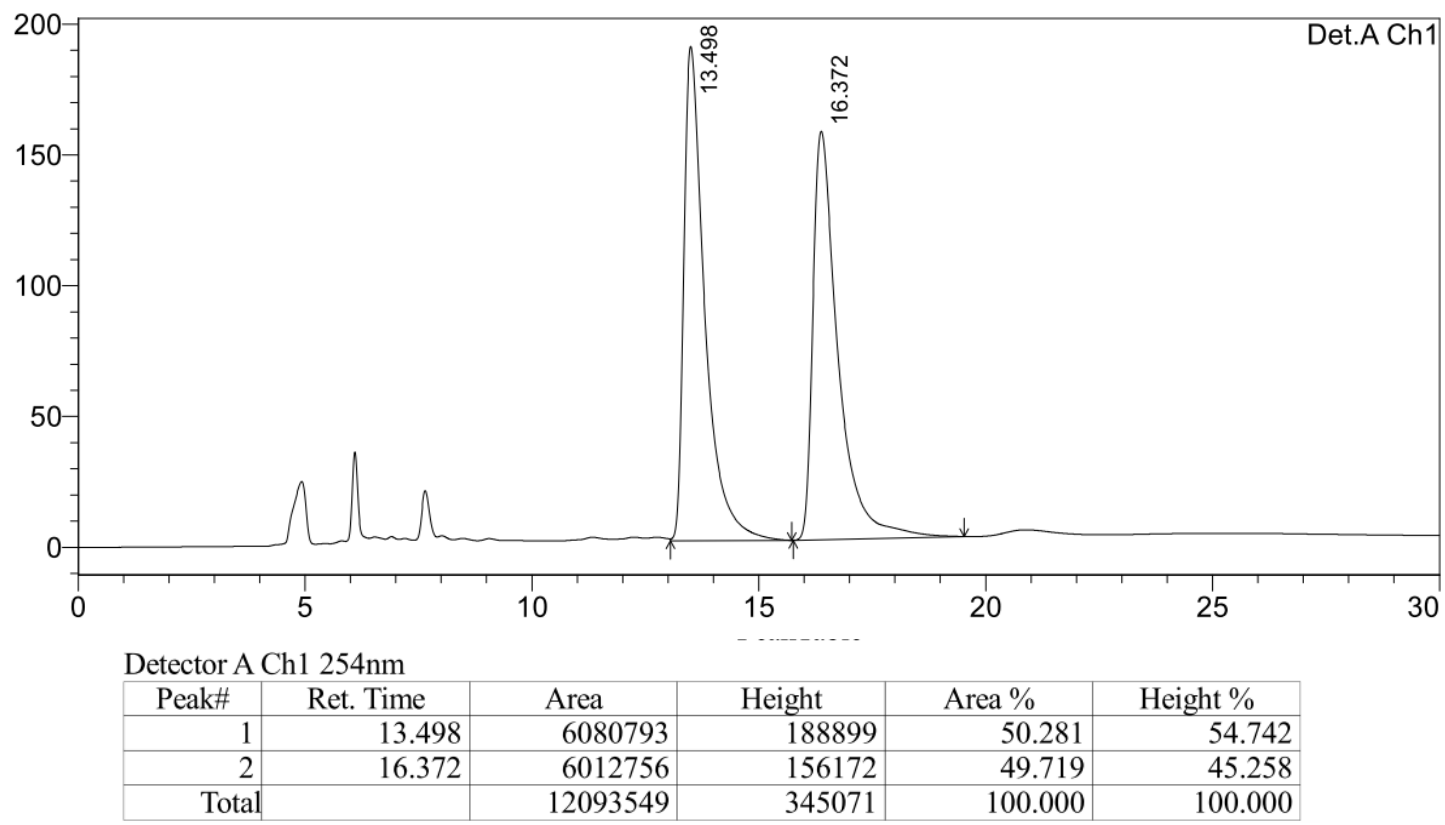

HPLC spectra of racemic reaction with palladium acetate and CPA-1

(HPLC analysis: IB column, $25^{\circ} \mathrm{C}$, hexans $/ \mathrm{iPrOH}=70 / 30,0.7 \mathrm{~mL} / \mathrm{min}, \lambda=254 \mathrm{~nm}$ )

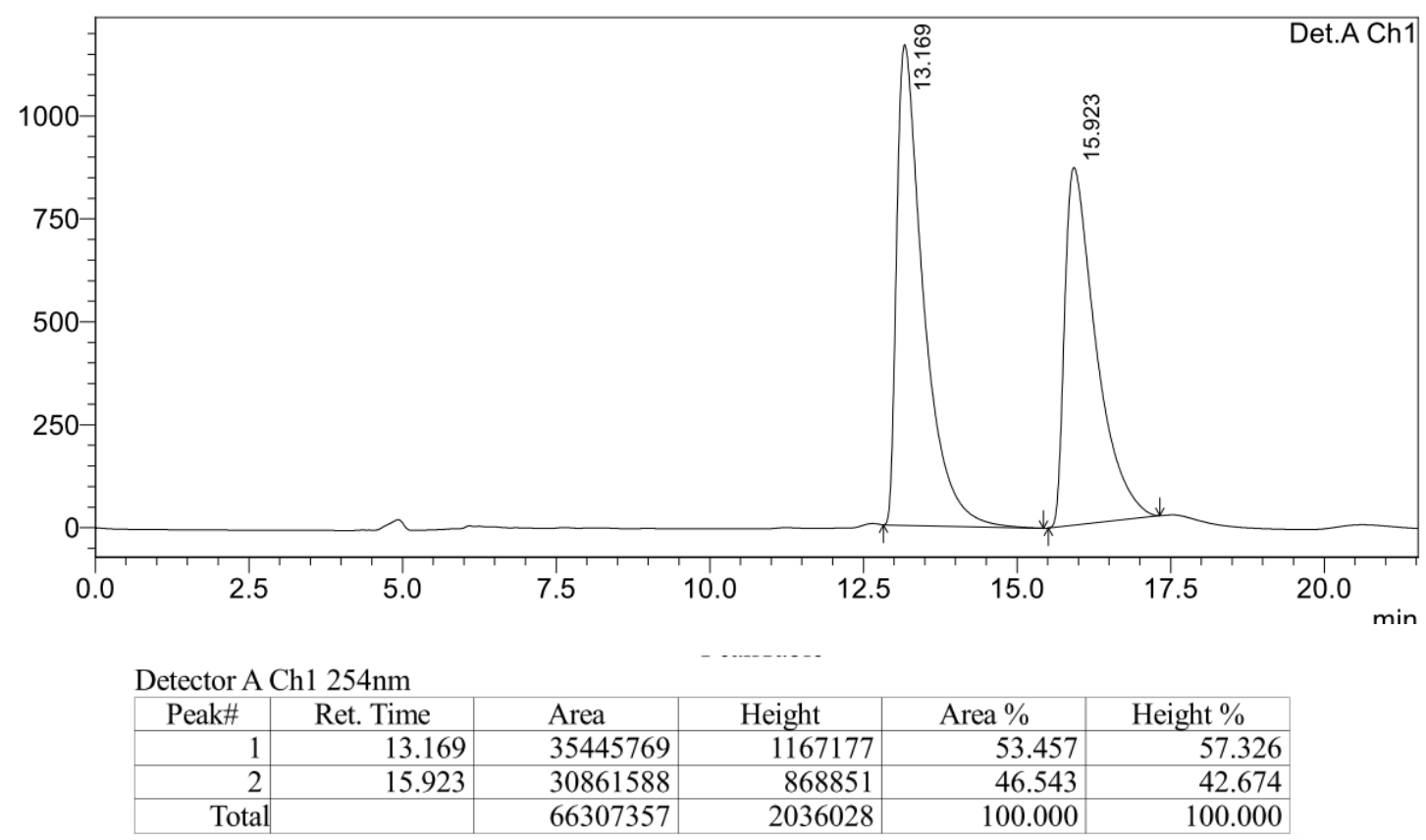


HPLC spectra of racemic reaction with palladium acetate and CPA-3

(HPLC analysis: IB column, $25^{\circ} \mathrm{C}$, hexans $/ \mathrm{iPrOH}=70 / 30,0.7 \mathrm{~mL} / \mathrm{min}, \lambda=254 \mathrm{~nm}$ )

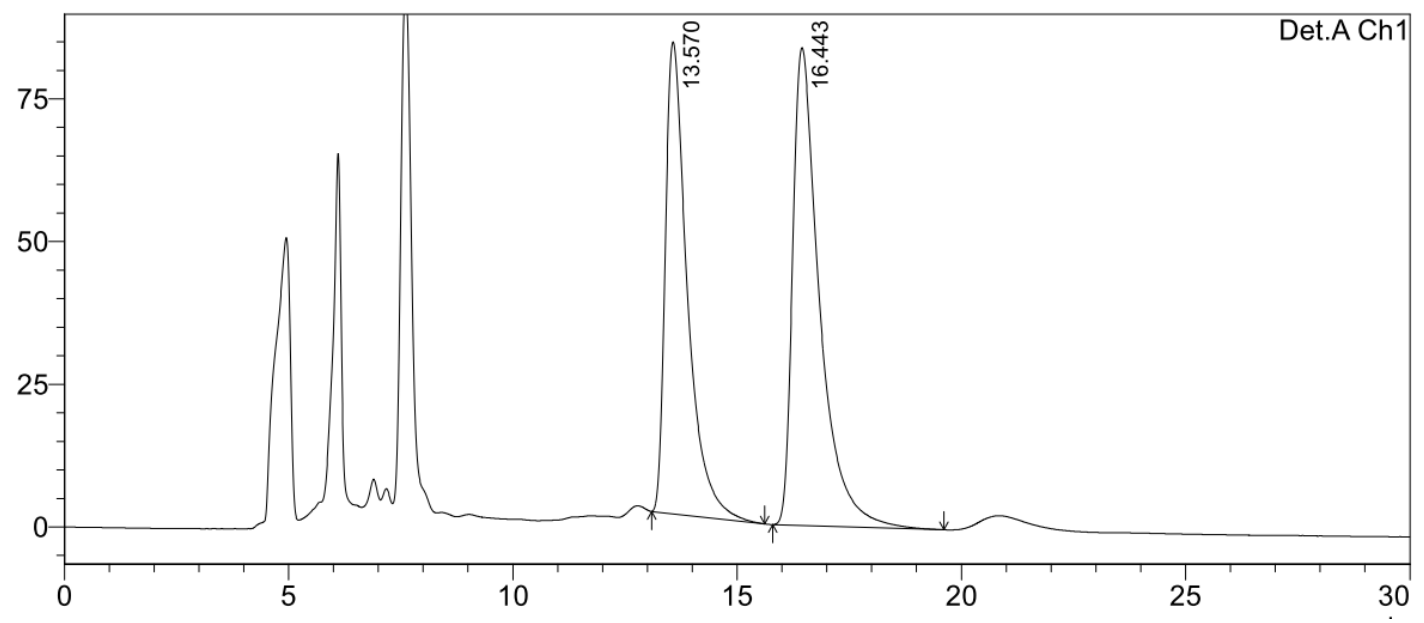

Detector A Ch1 254nm

\begin{tabular}{|r|r|r|r|r|r|}
\hline Peak\# & Ret. Time & \multicolumn{1}{|c|}{ Area } & \multicolumn{1}{|c|}{ Height } & Area \% & \multicolumn{1}{c|}{ Height \% } \\
\hline 1 & 13.570 & 2825987 & 82693 & 44.768 & 49.691 \\
\hline 2 & 16.443 & 3486577 & 83721 & 55.232 & 50.309 \\
\hline Total & & 6312564 & 166414 & 100.000 & 100.000 \\
\hline
\end{tabular}

\section{HPLC spectra of racemic reaction with palladium chloride/R-BINAP and TFA}

(HPLC analysis: IB column, $25^{\circ} \mathrm{C}$, hexans $/ \mathrm{iPrOH}=70 / 30,0.7 \mathrm{~mL} / \mathrm{min}, \lambda=254 \mathrm{~nm}$ )

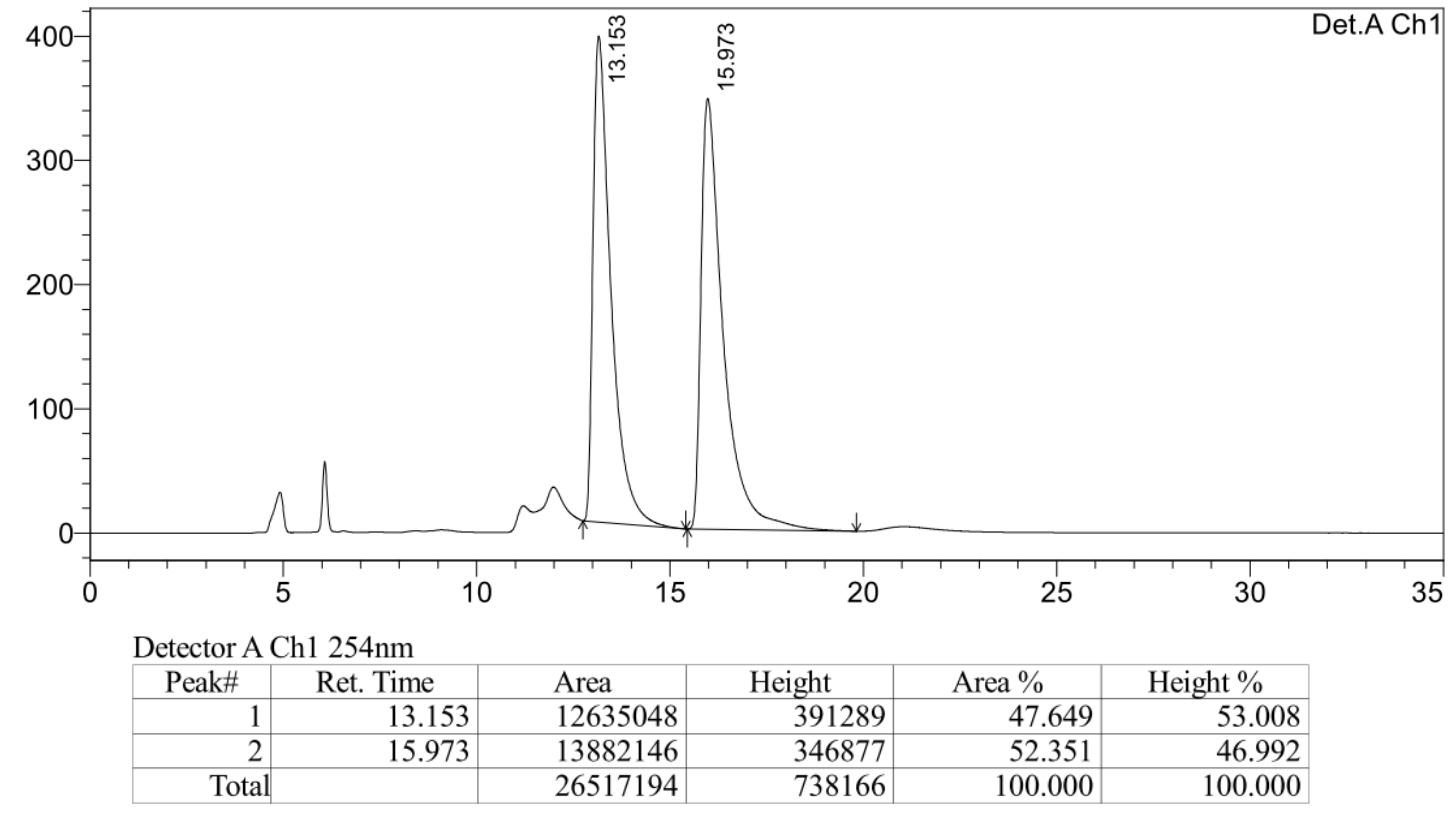

